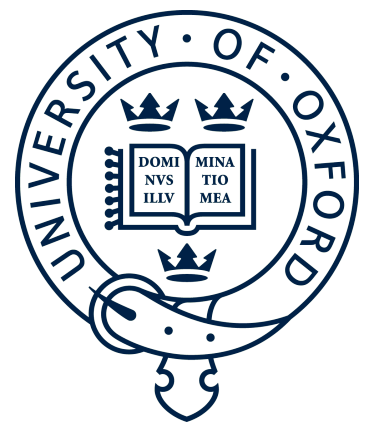

\title{
UNIVERSITY OF OXFORD
}

TRINity College

D.Phil. Mathematics

\section{Algebraic Topology of Manifolds}

Higher Orientability and Spaces of Nested Manifolds

Author:

Dr. Renee HoEkzema
Supervisor:

Prof. Ulrike TillmanN

August 30, 2018 



\title{
Algebraic Topology of Manifolds
}

\author{
Higher Orientability and Spaces of Nested Manifolds
}

\begin{abstract}
Part [ of this thesis concerns the question in which dimensions manifolds with higher orientability properties can have an odd Euler characteristic. In chapter 1 I prove that a $k$-orientable manifold (or more generally Poincaré complex) has even Euler characteristic unless the dimension is a multiple of $2^{k+1}$, where we call a manifold $k$-orientable if the $i^{\text {th }}$ Stiefel-Whitney class vanishes for all $0<i<2^{k}(k \geq 0)$. For $k=0,1,2,3, k$-orientable manifolds with odd Euler characteristic exist in all dimensions $2^{k+1} m$, but whether there exist a 4-orientable manifold with an odd Euler characteristic is an open problem. In Chapter 2 I present calculations on the cohomology of the first two Rosenfeld planes, revealing that $(\mathbb{O} \otimes \mathbb{C}) \mathbb{P}^{2}$ is 2-orientable and $(\mathbb{O} \otimes \mathbb{H}) \mathbb{P}^{2}$ is at least 3-orientable.

Part II discusses the homotopy type of spaces of nested manifolds. I prove that the space of $d$-dimensional manifolds with $k$-dimensional submanifolds inside $\mathbb{R}^{n}$ has the homotopy type of a linearised model $T_{k<d}$, which can be thought of as a space of off-set $d$-planes inside $\mathbb{R}^{n}$ with a (potentially empty) off-set $k$-plane inside of it, compactified with a point at infinity representing the empty set. Applying an induction I generalise this result to the case of higher nestings, establishing that the space $\Psi_{I}\left(\mathbb{R}^{n}\right)$ of nested manifolds inside $\mathbb{R}^{n}$, for $I$ a finite list of strictly increasing dimensions between 0 and $n-1$, has the homotopy type of a linearised model space $T_{I}$.
\end{abstract}





\section{Contents}

\begin{tabular}{ll}
\hline Abstract & 2
\end{tabular}

\begin{tabular}{ll}
\hline Extended Abstract & 7
\end{tabular}

I Manifolds with odd Euler characteristic and higher $\begin{array}{ll}\text { orientability } & 10\end{array}$

1 Manifolds with odd Euler characteristic and higher orientabil-

$\begin{array}{ll}\text { ity } & 11\end{array}$

1.1 Introduction . . . . . . . . . . . . . . . . . . 11

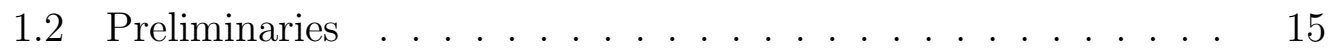

$1.2 .1 \quad$ Steenrod Algebra $\ldots \ldots \ldots \ldots$

1.2 .2 Stiefel-Whitney classes $\ldots \ldots \ldots \ldots$

$1.2 .3 \quad$ Wu classes . . . . . . . . . . . . . . . . . . . 18

1.3 Vanishing top Wu class implies even Euler characteristic ... 20

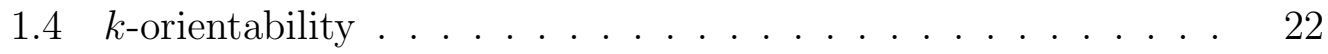

1.5 Proof of Theoreml1.2 $\ldots \ldots \ldots \ldots \ldots \ldots$

$\begin{array}{|ll|}2 & \text { Orientability of the Rosenfeld planes }\end{array}$ 
2.1 Introduction . . . . . . . . . . . . . . . . . . . . . . . . 32

$2.2 \quad$ Orientability of EIII or $(\mathbb{O} \otimes \mathbb{C}) \mathbb{P}^{2} \ldots \ldots \ldots \ldots$

$2.3 \quad$ Orientability of $E V I$ or $(\mathbb{O} \otimes \mathbb{H}) \mathbb{P}^{2} \ldots \ldots \ldots$

2.3.1 Computing the action of $S q^{3}$ on the generators. . . . . 44

2.3.2 Computing the action of $S q^{5}$ on the generators. . . . . 45

2.3 .3 Computing the action of $S q^{6}$ on the generators. . . . . 46

2.3 .4 Computing the action of $S q^{7}$ on the generators. . . . . 47

$2.3 .5 \quad 3$-orientability of $(\mathbb{O} \otimes \mathbb{H}) \mathbb{P}^{2} \ldots \ldots \ldots$

$2.3 .6 \quad$ Potential 4-orientability . . . . . . . . . . . . . 49

II Homotopy type of the space of nested manifolds 51

3 Overview of program of studying nested manifolds and cobordisms

3.1 Introduction and background to the study of nested manifolds and cobordisms . . . . . . . . . . . . . . . . 52

3.1 .1 Nested manifolds and cobordisms . . . . . . . . . . . 52

3.1 .2 Cobordism categories with additional structure . . . 55

3.1.3 Applications of nested cobordism categories . . . . . 56

3.2 Nested cobordism categories: the overarching program . . . . 59

3.3 How the space of manifolds relates to the cobordism category 62

4 Homotopy type of the space of nested manifolds 64

4.1 Introduction . . . . . . . . . . . . . . . . . . . . . . 64

4.2 Homotopy type of the space of embedded manifolds . . . . . 66

4.2 .1 Definitions . . . . . . . . . . . . . . 66 
4.2.2 Technical lemma concerning the size of tubular neighbourhoods ................ . 67

4.2 .3 Homotopy type of the space of manifolds . . . . . . . . 73

4.3 Homotopy type of spaces of once nested manifolds . . . . . . . 75

4.3.1 Technical lemma concerning the injectivity radius of the tangential exponential map . . . . . . . . . . . . . 75

4.3 .2 Definitions . . . . . . . . . . . . . . 78

4.3.3 Homotopy type of the space of once nested manifolds . 81

4.4 Homotopy type of spaces of arbitrarily nested manifolds. . . . 92

4.4 .1 Definitions . . . . . . . . . . . . . . . . . 92

4.4 .2 Homotopy type of spaces of nested manifolds . . . . . . 93

\begin{tabular}{|lr}
\hline Acknowledgements & 99
\end{tabular}

\begin{tabular}{ll}
\hline Bibliography & 103
\end{tabular} 


\section{Extended Abstract}

Part I of this thesis concerns the question in which dimensions manifolds with higher orientability properties can have an odd Euler characteristic. It is wellknown that odd-dimensional manifolds have Euler characteristic zero. Furthermore, orientable manifolds have an even Euler characteristic unless the dimension is a multiple of 4 . In chapter 1 I give a generalisation of these statements: a $k$-orientable manifold (or more generally Poincaré complex) has even Euler characteristic unless the dimension is a multiple of $2^{k+1}$, where we call a manifold $k$-orientable if the $i^{\text {th }}$ Stiefel-Whitney class vanishes for all $0<i<2^{k}$ $(k \geq 0)$. More generally, I show that for a $k$-orientable manifold the Wu classes $v_{l}$ vanish for all $l$ that are not a multiple of $2^{k}$. This work has been accepted for publication in International Mathematical Research Notices, see [Hoe17]. For $k=0,1,2,3, k$-orientable manifolds with odd Euler characteristic exist in all dimensions $2^{k+1} m$, but whether there exist a 4-orientable manifold with an odd Euler characteristic is an open problem. In Chapter 2 this question is approached by discussing ways to find such a manifold. In particular I present calculations determining the action of certain Steenrod operations on the cohomology of two manifolds with odd Euler characteristic and dimensions high powers of two, in an effort to determine their orientability. These calculations reveal that the 32-dimensional manifold known as the first Rosenfeld plane $(\mathbb{O} \otimes \mathbb{C}) \mathbb{P}^{2}$, or alternatively in Cartan's classification of symmetric spaces as $E I I I$, is 2-orientable but not 3-orientable. Furthermore, the 64-dimensional second Rosenfeld plane $(\mathbb{O} \otimes \mathbb{H}) \mathbb{P}^{2}$, also known as $E V I$, is 3-orientable, and a condition is presented under which it is 4-orientable. This can at present 
not be resolved, hence the question whether a 4-orientable manifold with odd Euler characteristic exists remains open.

Part III of this thesis discusses manifolds from a different point of view. We define a nested manifold as a manifold endowed with a submanifold, which is potentially endowed with a submanifold of its own, et cetera. We establish the homotopy type of spaces of nested manifolds inside $\mathbb{R}^{n}$, with an eye towards determining the homotopy type of a category of nested cobordisms. This is in analogy to the work of Galatius, Madsen, Tillmann and Weiss establishing the homotopy type of the cobordism category in [GMTW09], and refinements of this result by Galatius and Randal-Williams for the case of a cobordism category subordinate to $\mathbb{R}^{n}$ in [GRW10]. Chapter 3 discusses the motivation and background to this work, as well as setting up the overall structure of the ongoing program into which the results in this thesis fit.

Chapter 4 discusses the homotopy type of spaces of nested manifolds, with a particular emphasis on technical details in differential geometry that lie at the foundation of these sorts of proofs. Section 4.2 discusses the original proof in [GRW10] that the homotopy type of the space of $d$-dimensional manifolds inside $\mathbb{R}^{n}$ is captured by a linearised model given by the Thom space of the anticanonical bundle over the Grassmannian of $d$-dimensional planes inside $\mathbb{R}^{n}$. In particular I highlight a new technical assumption concerning a continuous choice of tubular neighbourhood on the space of manifolds that is added to elucidate the proof.

Section 4.3 discusses the case of the space $\Psi_{k<d}\left(\mathbb{R}^{n}\right)$ of $d$-dimensional manifolds inside $\mathbb{R}^{n}$ endowed with $k$-dimensional submanifolds. First I describes an additional technical assumption concerning a continuous choice of a radius 
inside of which the tangential exponential map of the manifold at 0 is injective. Subsequently a once-nested linearised model $T_{k<d}$ is defined, which can be thought of as a space of off-set $d$-planes inside $\mathbb{R}^{n}$ with a (potentially empty) off-set $k$-plane inside of it, compactified with a point at infinity representing the empty set. I then prove that this space is indeed weakly homotopy equivalent to the space $\Psi_{k<d}\left(\mathbb{R}^{n}\right)$. Section 4.4 applies an induction to generalise this result to the case of higher nestings, establishing that the space $\Psi_{I}\left(\mathbb{R}^{n}\right)$ of nested manifolds inside $\mathbb{R}^{n}$, for $I$ a finite list of strictly increasing dimensions between 0 and $n-1$, has the homotopy type of a linearised model space $T_{I}$. 


\title{
Part I
}

\section{Manifolds with odd Euler}

characteristic and higher

\author{
orientability
}




\section{Chapter 1}

\section{Manifolds with odd Euler characteristic and higher orientability}

\subsection{Introduction}

Manifolds are central objects of study in geometry and topology. A basic property of a manifold is whether it is orientable. If an $n$-dimensional manifold $M$ is closed and connected, then $M$ is orientable if and only if its $n^{\text {th }}$ homology group $H_{n}(M ; \mathbb{Z})$ is isomorphic to $\mathbb{Z}$. An orientation on $M$ is a choice of a generator for this group. A manifold is also orientable if and only if the first Stiefel-Whitney class $w_{1}$ of the tangent bundle of $M$ vanishes. This characteristic class is the obstruction to lifting the classifying map $\tau: M \rightarrow B O(n)$ of the tangent bundle to $B S O(n)$, which is the double cover of $B O(n)$.

A more restrictive property of a manifold, extending orientability, is spinability. 
We call a manifold spinable if $\tau$ admits a lift to $B \operatorname{Spin}(n)$, the 3-connected cover of $B O(n)$. For an orientable manifold the obstruction for this lift is given by the second Stiefel-Whitney class $w_{2}$. Hence a manifold is spinable precisely if $w_{1}=w_{2}=0$.

Orientability and spinability can be generalised to a sequence of higher orientability conditions in different ways, of which we here discuss three. The first two are well-studied, whereas the third notion is new. The first generalisation of orientability is given by the property of being $k$-parallelizable. A manifold is $k$-parallelizable if the classifying map of the tangent bundle $\tau$ can be lifted to the $k$-connected cover of $B O(n)$. These connected covers form the Whitehead tower of $B O(n)$, of which each stage successively kills a higher homotopy group. By Bott periodicity, $B O=\lim _{n} B O(n)$ has a non-trivial homotopy group in dimensions $0,1,2,4(\bmod 8)$, and therefore non-trivial stages of the Whitehead tower occur only in these dimensions. The first few non-trivial stages have special names:

$$
B O(n) \leftarrow B S O(n) \leftarrow B \operatorname{Spin}(n) \leftarrow B \operatorname{String}(n) \leftarrow B \text { brane }(n) \leftarrow \ldots
$$

For example, BString $(n)$ is the stage at which the fourth homotopy group is killed, and it is 7-connected. If $\tau$ admits a lift to $\operatorname{BString}(n)$, we call the manifold stringable, which corresponds to 7-parallelizable. For a spinable manifold, this requires $\tau$ to be trivial on the fourth homotopy group, which is a strictly stronger condition than requiring that $w_{4}=0$. The obstruction class to finding a string structure on a spin manifold is $\frac{p_{1}}{2} \in H^{4}(M ; \mathbb{Z})$. If a manifold is $k$-parallizable for $k=n$, the dimension of the manifold, then the manifold is parallelizable or frameable. 
A second notion of "higher" orientations arises from considering orientability with respect to different (co)homology theories (see for example [Rud98], chapter V). There are occasionally links between the notion of being $k$-parallelizable and being orientable with respect to a certain cohomology theory. For example, a smooth manifold admits an orientation in real $K$-theory if and only if it is spinable $([\mathrm{ABS64}])$. If a manifold is stringable, then it admits an orientation for $\operatorname{tm} f$, the universal elliptic cohomology theory ([AHR]).

We now define a third sequence of orientability conditions that can also be viewed as a natural generalization of orientability and spinability:

Definition 1.1. We call a manifold $k$-orientable if $w_{i}=0$ for $0<i<2^{k}$.

A manifold is 1-orientable if and only if it is orientable. A manifold is 2orientable if and only if it is spinable. If a manifold is stringable, then it is 3-orientable, but the converse is not true. A counterexample is given by the manifold $\mathbb{C P}^{3}$, all of whose Stiefel-Whitney classes vanish although it is not stringable ([DHH10]). Generally, increasing parallelizability by one non-trivial step in the Whitehead tower implies increasing orientability by one, but the first poses a strictly stronger condition (for details see Lemma 1.12.

Orientability bears a nice relationship to a very classical invariant of topological spaces, the Euler characteristic $\chi$. It is a simple consequence of Poincaré duality that manifolds with an odd dimension have vanishing $\chi$. For orientable manifolds however, $\chi$ is furthermore even in dimensions that are not a multiple of 4. This pattern continues: spinable manifolds have an even $\chi$ in dimensions that are not a multiple of 8 . This is implied by Ochanine's theorem on the divisibility of the signature of spin manifolds in dimension $8 k+4$ ([Och81]). 
The generalisation of these statements to higher $k$-orientability is the main result of this chapter:

Theorem 1.2 (Corollary 1.16). A k-orientable manifold or Poincaré complex $M(k \geq 0)$ has an even Euler characteristic $\chi(M)$ if its dimension is not a multiple of $2^{k+1}$.

This theorem follows as a corollary from the following stronger statement about the vanishing of a large number of $\mathrm{Wu}$ classes.

Theorem 1.3 (Theorem 1.15). Let $M^{n}$ be a manifold or Poincaré complex of dimension $n$. If $M$ is $k$-orientable for some $k$, then the $W u$ classes $v_{l}$ vanish for all $l$ such that $2^{k} \nmid l$.

The proof is based on a relation within the Steenrod algebra derived from recursive application of the Adem relations.

In the 50's and 60's of the last century, the problem of relations between Stiefel-Whitney classes of manifolds was studied in [Dol56, Mas60, BP64]. The question posed was to find all polynomials in Stiefel-Whitney classes of homogeneous cohomological degree that vanish for all manifolds of a given dimension $n$. Dold answered this question for degree $n$ polynomials, and Brown and Peterson gave a complete although indirect description of this set of polynomials for arbitrary degree in terms of a right action of the Steenrod algebra on the cohomology of $B O$. In the current work, we partially answer the subtly different question "if $w_{i}=0$ for $0<i<2^{k}$, does $w_{n}$ vanish?" Indeed, Brown and Peterson found an extended set of relations when considering the case of orientable manifolds. It would be interesting to study the more general question "if $w_{i}=0$ for $0<i<2^{k}$, which $w_{j}$ can be non-vanishing?", as well as to make the relations found in [BP64] more explicit. 
Theorem 1.2 in particular implies that 3-orientable and therefore stringable manifolds have an even Euler characteristic unless the dimension is a multiple of 16 , that 4-orientable and therefore 5braneable manifolds have an even $\chi$ unless the dimension is a multiple of 32 , et cetera. One might wonder whether the theorem is strict: whether $k$-orientable manifolds with an odd Euler characteristic exist in all dimensions that are a multiple of $2^{k+1}$. There are non-orientable manifolds with odd $\chi$ in every even dimension as $\chi\left(\mathbb{R} \mathbb{P}^{2 m}\right)=1$. Similarly, even-dimensional complex projective spaces $\mathbb{C P}^{2 m}$, even-dimensional quaternion projective spaces $\mathbb{H} \mathbb{P}^{2 m}$, and powers of the octonion projective plane or Cayley plane $\left(\mathbb{O P}^{2}\right)^{m}$ are examples of manifolds with an odd Euler characteristic that are orientable and of dimension $4 m$, spinable of dimension $8 m$, and stringable of dimension $16 m$ respectively. Hence the theorem is strict for $k=0,1,2,3$. However, to the author's knowledge, it is an open question whether there exist 4-orientable manifolds with an odd Euler characteristic. In particular, one might wonder whether there exist 8-connected manifolds with an odd Euler characteristic.

\section{Conventions}

Throughout this chapter we will be considering (co)homology with $\mathbb{Z} / 2$ coefficients, hence from here on we write $H^{n}(X)$ for $H^{n}(X ; \mathbb{Z} / 2)$. Manifolds are considered to be closed and without boundary.

\subsection{Preliminaries}

This section recalls definitions and some properties of Steenrod squares, StiefelWhitney classes and Wu classes. 


\subsubsection{Steenrod Algebra}

Steenrod squares are cohomology operations on $\mathbb{Z} / 2$ cohomology. We recall from [SE62] their axiomatic definition:

1. $S q^{i}: H^{n}(X) \rightarrow H^{n+i}(X)$ are cohomology operations, i.e. they are natural with respect to maps of spaces.

2. $S q^{0}$ is the identity homomorphism.

3. $S q^{|x|}(x)=x \smile x$.

4. If $i>|x|$, where $|x|$ is the degree of $x$, then $S q^{i}(x)=0$.

5. The Steenrod squares obey the Cartan formula:

$$
S q^{k}(x \smile y)=\sum_{i+j=k} S q^{i}(x) \smile S q^{j}(y)
$$

It follows from these axioms that the Steenrod squares obey the Adem relations: for all $a$ and $b$ such that $a<2 b$, we have

$$
S q^{a} S q^{b}=\sum_{c=0}^{\lfloor a / 2\rfloor}\left(\begin{array}{c}
b-c-1 \\
a-2 c
\end{array}\right) S q^{a+b-c} S q^{c}
$$

As a result of the Adem relations, certain composites of Steenrod squares always vanish, for example $S q^{2 n-1} S q^{n}=0$, as for $a=2 n-1$ and $b=n$ all binomials on the right hand side of the Adem relations vanish. Another consequence of the Adem relations is that individual squares can be decomposed into a sum of composites of smaller squares, unless they are of the form $S q^{2^{i}}$ 
for some $i$. Steenrod squares, subject to the Adem relations, generate a graded Hopf algebra over $\mathbb{F}_{2}$ called the Steenrod algebra $\mathcal{A}$.

One often groups the Steenrod operations into the total Steenrod square acting on $\bigoplus_{i} H^{i}(X)$ given by

$$
S q=S q^{0}+S q^{1}+S q^{2}+\ldots
$$

\subsubsection{Stiefel-Whitney classes}

Stiefel-Whitney classes are $\mathbb{Z} / 2$ characteristic classes of vector bundles. We recall some properties from May99, Chapter 23. An $n$-dimensional vector bundle $\mathbb{R}^{n} \rightarrow \xi \rightarrow M$ over a manifold $M$ can be viewed as the pull-back of the universal bundle $\mathbb{R}^{n} \rightarrow \gamma_{n} \rightarrow B O(n)$ under a classifying map which we shall also denote $\xi$.

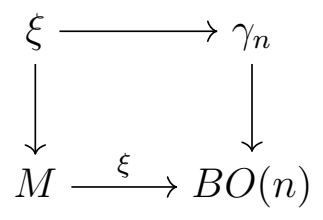

The $\mathbb{Z} / 2$ cohomology of $B O(n)$ is given by:

$$
H^{*}(B O(n))=\mathbb{Z} / 2\left[w_{1}, w_{2}, \ldots, w_{n}\right]
$$

where $w_{i}$ with $\left|w_{i}\right|=i$ are the universal Stiefel-Whitney classes. We can ask what the image of this cohomology is under the classifying map $\xi$.

Definition 1.4. Given a vector bundle $\xi$ over a manifold $M$, the $i$-th StiefelWhitney class $w_{i}(\xi) \in H^{i}(M)$ is defined as

$$
w_{i}(\xi)=\xi^{*}\left(w_{i}\right)
$$


for $w_{i} \in H^{*}(B O)$ the universal $i$-th Stiefel-Whitney class.

By the Stiefel-Whitney classes of a manifold we mean the Stiefel-Whitney classes of its tangent bundle. The Stiefel-Whitney classes of a manifold obey the $\mathrm{Wu}$ formula:

$$
S q^{i}\left(w_{j}\right)=\sum_{t=0}^{i}\left(\begin{array}{c}
j+t-i-1 \\
t
\end{array}\right) w_{i-t} w_{j+t} .
$$

\subsubsection{Wu classes}

Let $M$ be an $n$-dimensional Poincaré complex (see for example [Ran02]) with fundamental class $\mu \in H_{n}(M)$ such that

$$
-\cap \mu: H^{*}(M) \rightarrow H_{n-*}(M)
$$

is an isomorphism. A topological manifold is a particular example of a Poincaré complex, as is any finite CW complex that is homotopy equivalent to a manifold.

Let

$$
\langle., .\rangle: H^{i}(M) \times H_{i}(M) \rightarrow \mathbb{Z} / 2
$$

be the pairing of cohomology and homology. $M$ satisfies Poincaré duality for $\mathbb{Z} / 2$ (co)homology, hence we have

$$
\operatorname{Hom}\left(H^{n-k}(M), \mathbb{Z} / 2\right) \cong H_{n-k}(M) \cong H^{k}(M),
$$

where a cohomology class $y \in H^{k}(M)$ corresponds to the homomorphism $x \mapsto\langle y \smile x,[M]\rangle$. In particular, one element of $\operatorname{Hom}\left(H^{n-k}(M), \mathbb{Z} / 2\right)$ is given 
by $x \mapsto\left\langle S q^{k}(x),[M]\right\rangle$. Under the above isomorphism, this corresponds to a degree $k$ cohomology class $v_{k}$ called the $k$-th Wu class, with

$$
\left\langle v_{k} \smile x,[M]\right\rangle=\left\langle S q^{k}(x),[M]\right\rangle,
$$

for all $x \in H^{n-k}(M)$. We can define the total Wu class as the formal sum

$$
v=1+v_{1}+v_{2}+\ldots+v_{n}
$$

This allows us to replace equation $(1.9)$ by

$$
\langle v \smile x,[M]\rangle=\langle S q(x),[M]\rangle
$$

where $S q$ is the total Steenrod square and $x \in H^{*}(M)$.

Because $S q^{i}(x)=0$ if $i>|x|$, an $n$-dimensional manifold only admits non-zero Steenrod operations for $i \leq \frac{n}{2}$. Hence a manifold has maximally $\frac{n}{2}$ non-zero Wu classes.

For a smooth manifold $M$ of dimension $n$, the Stiefel-Whitney classes of the manifold can be determined from the Steenrod squares and Wu classes. Define the total Stiefel-Whitney class as the formal sum

$$
w=1+w_{1}+w_{2}+\ldots+w_{n} .
$$

The total Stiefel-Whitney class $w$ is then determined by the total Steenrod square $S q$ and the total Wu class $v$ via the relation (see for example May99, 
Chapter 23):

$$
w=S q(v)
$$

This formula can be used to define Stiefel-Whitney classes for a Poincaré complex.

\subsection{Vanishing top Wu class implies even Euler characteristic}

As a key step in the proof of Theorem 1.3 we will need the statement that the vanishing of the top $\mathrm{Wu}$ class of an even-dimensional manifold or Poincaré complex implies that the Euler characteristic is even. The vanishing of the top $\mathrm{Wu}$ class is in fact a stronger condition than having an even Euler characteristic. This is a classical result, but will be recalled in this section.

Theorem 1.5 ([MS74]). For a smooth compact manifold $M^{n}$, the top StiefelWhitney class $w_{n}$ is the modulo 2 reduction of an integral characteristic class called the Euler class and it follows that the Stiefel-Whitney number $\left\langle w_{n},[M]\right\rangle$ equals $\chi(M)$ modulo 2 , hence $w_{n}=0$ if and only if $\chi(M)$ is even.

For a manifold of even dimension $2 n$, equation 1.13 implies that the top StiefelWitney class $w_{2 n}=S q^{n} v_{n}=v_{n}^{2}$, hence $v_{n}=0$ implies $w_{2 n}=0$, which implies that the Euler characteristic is even.

In the more general case of a Poincaré complex, one can define Stiefel-Whitney classes via the $\mathrm{Wu}$ formula. One can then show that it still holds that the top Stiefel-Whitney class $w_{2 n}$ is zero if and only if the Euler characteristic is even. We here take a different route to illustrate an alternative proof method. In 
order to avoid the use of the Euler class we will prove that the vanishing of $v_{n}$ implies an even Euler characteristic directly. For this we will first give a proof of the statement that a symplectic vector space over any field, in particular also $\mathbb{F}_{2}$, has even dimension. This statement is very classical (see for example [Jac74], section 6.2). We call a vector space $V$ over a field $k$ symplectic if it is endowed with a bilinear form $A: V^{n} \times V^{n} \rightarrow k$ that is non-degenerate, antisymmetric and has vanishing diagonal. The last condition is superfluous if $\operatorname{char}(k) \neq 2$.

Lemma 1.6. Let $V^{n}$ be an $n$-dimensional vector space over a field $k$ and $A: V^{n} \times V^{n} \rightarrow k$ an antisymmetric bilinear form with vanishing diagonal, i.e.

$$
A(v, v)=0 \quad \forall v \in V^{n} .
$$

If $n$ is odd, then $A$ is degenerate i.e. $\operatorname{det} A=0$.

Proof. Consider the formula for the determinant:

$$
\operatorname{det}(A)=\sum_{\sigma \in S_{n}} \operatorname{sgn}(\sigma) \prod_{i=1}^{n} a_{i, \sigma(i)},
$$

where $S_{n}$ is the symmetric group on $n$ letters and $a_{i, j}$ are the matrix elements of $A$ in a given basis of $V^{n}$. As the diagonal of $A$ expressed as a matrix in this basis vanishes, any permutation $\sigma$ that leaves one or more indices unchanged does not contribute to the sum. Hence, we can restrict to the set of permutations $\Sigma_{n}$ containing no cycles of length 1 . Within this subset, an element is of order two (its own inverse) if and only if it consists of disjoint cycles of length two. Since $n$ is odd, there us no such element. Hence $\Sigma_{n}$ can be partitioned as $\Sigma_{n}^{+} \sqcup \Sigma_{n}^{-}$such that $\Sigma_{n}^{-}$consists of the inverses of the elements 
in $\Sigma_{n}^{+}$. The determinant formula then becomes:

$$
\operatorname{det}(A)=\sum_{\sigma \in \Sigma_{n}^{+}} \operatorname{sgn}(\sigma) \prod_{i=1}^{n} a_{i, \sigma(i)}+\operatorname{sgn}\left(\sigma^{-1}\right) \prod_{i=1}^{n} a_{i, \sigma^{-1}(i)}
$$

Note that $\operatorname{sgn}(\sigma)=\operatorname{sgn}\left(\sigma^{-1}\right)$. Furthermore, as $A$ is antisymmetric and $n$ odd, $\prod_{i=1}^{n} a_{i, \sigma^{-1}(i)}=-\prod_{i=1}^{n} a_{\sigma^{-1}(i), i}=-\prod_{i=1}^{n} a_{i, \sigma(i)}$, by rearranging the terms in the product. Hence the two products in equation (1.16) cancel and hence $\operatorname{det}(A)=0$.

Theorem 1.7. Let $M^{2 n}$ be a Poincaré complex of dimension $2 n$. Then $v_{n}=0$ implies that $\chi(M)$ is even.

Proof. Define the (anti-)symmetric bilinear form

$$
\omega: H^{n}(X) \times H^{n}(X) \rightarrow \mathbb{Z} / 2
$$

given by $\omega(x, y)=\langle(x \smile y), \mu\rangle$.

As $x^{2}=S q^{n}(x)=v_{n} \smile x=0$ for all $x \in H^{n}(X)$, the diagonal of the $\omega$ is zero. It is furthermore non-degenerate because of Poincaré duality and because we are working over a field. Therefore $H^{n}(X)$ is a symplectic vector space, and thus has even dimension by Lemma 1.6. The parity of the Euler characteristic of a Poincaré complex is determined by the parity of the dimension of the middle cohomology, hence it is even.

\section{$1.4 k$-orientability}

A manifold is called $k$-orientable if $w_{i}=0$ for $0<i<2^{k}$. This definition is motivated by [DHH10], where it is noted that the condition $w_{i}=0$ for 
$0<i<2^{k}$ for a manifold $M$ is equivalent to the condition that $H^{*}(M)$ is Poincaré dual with respect to the subalgebra $\mathcal{A}(k-1)$ of the Steenrod algebra spanned by $S q^{1}, S q^{2}, \ldots, S q^{2^{k-1}}$. The latter means that the actions of these squares are symmetrically distributed upon reflection in the middle dimension, where we should take every operation to its image under the canonical antihomomorphism $\chi$ from $\mathcal{A}(k)$ to itself (see [DHH10] for a detailed explanation). The well-known fact that the Stiefel-Whitney classes of degree a power of two generate all Stiefel-Whitney classes of a manifold is a consequence of the $\mathrm{Wu}$ formula and Lemma 1.10. The latter we will also need for the proof of the main theorem, hence we include both proofs.

Lemma 1.8. The Stiefel-Whitney classes of a smooth manifold are generated by those of the form $w_{2^{i}}$.

Lemma 1.8 implies that a $k$-orientable smooth manifold is $k+1$-orientable precisely if the class $w_{2^{k}}$ vanishes. This motivates why in the definition of $k$-orientability, it is natural to let the number of vanishing Stiefel-Whitney classes double as we increase $k$ by one. It also follows that in order for a smooth manifold to be $k$-orientable it is enough to require $w_{i}$ to vanish for $i \leq 2^{k-1}$.

Corollary 1.9. A smooth manifold is $k$-orientable if and only if $w_{i}=0$ for $0<i \leq 2^{k-1}$.

To prove Lemma 1.8, we need the following combinatorial lemma.

Lemma 1.10.

$$
\left(\begin{array}{c}
2^{k} m-1 \\
b
\end{array}\right) \equiv 1(\bmod 2)
$$

for all $0 \leq b \leq 2^{k-1}$ and $m, k \geq 1$. 
Proof. By Lucas's theorem ([Luc78]), the value of a binomial $\left(\begin{array}{l}a \\ b\end{array}\right)$ modulo a prime $p$ can be calculated by considering the base $p$ expansions of $a$ and $b$ :

$$
\left(\begin{array}{l}
a \\
b
\end{array}\right) \equiv \prod_{i}\left(\begin{array}{l}
a_{i} \\
b_{i}
\end{array}\right)(\bmod p)
$$

where $a_{i}$ and $b_{i}$ are the corresponding base $p$ digits of $a$ and $b$, and we use the convention that $\left(\begin{array}{l}a_{i} \\ b_{i}\end{array}\right)=0$ if $a_{i}<b_{i}$.

Consider the case $p=2$ with $a=2^{k} m-1$ and $b \leq 2^{k-1}$. Let $m=\sum_{i} m_{i} 2^{i}$ be the binary expansion of $m$, and $i_{0}$ the index of the lowest non-zero digit of $m$. Then

$$
a=2^{k} m-1=\sum_{i} m_{i} 2^{i+k}-1=\sum_{i>i_{0}} m_{i} 2^{i+k}+2^{i_{0}+k}-1,
$$

hence the binary expansion of $a$ ends in a sequence of 1's: $a_{i}=1$ for $0 \leq$ $i \leq i_{0}+k-1$. On the other hand, $b_{i}=0$ for $i \geq k$. Hence every term in equation 1.18 is of the form $\left(\begin{array}{l}1 \\ 0\end{array}\right),\left(\begin{array}{l}1 \\ 1\end{array}\right)$ or $\left(\begin{array}{l}0 \\ 0\end{array}\right)$, each of which equals 1 and hence $\left(\begin{array}{l}a \\ b\end{array}\right) \equiv 1(\bmod 2)$.

Proof of Lemma 1.8. We can rearrange the $\mathrm{Wu}$ formula 1.20 as follows:

$$
\left(\begin{array}{c}
j-1 \\
i
\end{array}\right) w_{j+i}=S q^{i}\left(w_{j}\right)+\sum_{t=0}^{i-1}\left(\begin{array}{c}
j+t-i-1 \\
t
\end{array}\right) w_{i-t} w_{j+t} .
$$

Hence we can rewrite $w_{n}$ in terms of Stiefel-Whitney classes of a lower degree precisely if we can split up $n$ as $i+j$ with $i, j \geq 1$ in such a way that $\left(\begin{array}{c}j-1 \\ i\end{array}\right)$ is non-zero modulo 2. If $n$ is not a power of 2 , then $n=2^{k}+i$ for some $i<2^{k}$, setting $j=2^{k}$. Applying Lemma 1.10 for $m=1$ and $b=i$, we see that $\left(\begin{array}{c}j-1 \\ i\end{array}\right) \equiv 1(\bmod 2)$. 
If $n$ is a power of 2 , then $i+j-1$ has only 1 's in its binary expansion, hence every factor in the product given by equation 1.18 computing $\left(\begin{array}{c}j-1 \\ i\end{array}\right)$ modulo 2 is of the form $\left(\begin{array}{l}1 \\ 0\end{array}\right) \equiv 1$ or $\left(\begin{array}{l}0 \\ 1\end{array}\right) \equiv 0$. Then the only choice of $i$ for which $\left(\begin{array}{c}j-1 \\ i\end{array}\right)$ modulo 2 is non-zero is then $i=0$, in which case the $\mathrm{Wu}$ formula states $w_{n}=w_{n}$.

The lemma below shows that we could also have used the $\mathrm{Wu}$ classes rather than the Stiefel-Whitney classes to define $k$-orientability.

Lemma 1.11. The following are equivalent:

(i) A manifold is k-orientable

(ii) $v_{i}=0$ for $0<i \leq 2^{k-1}$

(iii) $v_{i}=0$ for $0<i<2^{k}$

Proof. It follows from equation (1.13) that

$$
w_{i}=\sum_{j=0}^{\lfloor i / 2\rfloor} S q^{j}\left(v_{i-j}\right) .
$$

From this formula it follows immediately that $v_{i}=0$ for $i \leq 2^{k-1}$ implies $w_{i}=0$ for $i \leq 2^{k-1}$, and that $v_{i}=0$ for $i<2^{k}$ implies $w_{i}=0$ for $i<2^{k}$, giving the implications $(i i) \Rightarrow(i)$ and $(i i i) \Rightarrow(i)$.

We will show by induction that the lowest non-vanishing Stiefel-Whitney class equals the lowest non-vanishing $\mathrm{Wu}$ class, i.e. if $w_{i}=0$ for $0<i<n$, then $w_{i}=v_{i}$ for $i \leq n$. Note that $w_{1}=v_{1}$. Assume that $w_{i}=0$ for $0<i<n$ and 
by induction hypothesis $v_{i}=w_{i}=0$ for $0<i \leq n-1$. Then

$$
w_{n}=v_{n}+\sum_{j=1}^{\lfloor i / 2\rfloor} S q^{j}\left(v_{i-j}\right)=v_{n} .
$$

It follows that $w_{i}=0$ for $i<n$ implies $v_{i}=0$ for $i<n$, hence $(i) \Rightarrow(i i)$ and $(i) \Rightarrow(i i i)$

When comparing $k$-orientability to $k$-parallelizability, one would naively expect that only $2^{k-1}$-parallelizability would imply $k$-orientability of a manifold. However, by the classical work of Stong ([Sto63]), the Stiefel-Whitney classes of the $n$-connective covers of $B O$ vanish exponentially with $n$, with the number of vanishing $w_{i}$ doubling at each non-trivial stage (occurring in degrees $0,1,2$ and 4 modulo 8). Indeed, we have the following theorem:

Theorem 1.12 (Sto63]). Let $\phi(n)$ be the number of integers $s$ with $1 \leq s \leq n$ such that $s \equiv 0,1,2,4(\bmod 8)$. If a manifold $M$ is $n$-parallelizable for $n$ with $\phi(n) \geq k$, then $M$ is k-orientable.

In other words, a lift of the classifying map of the tangent space of $M$ to the $k^{\text {th }}$ non-trivial stage in the Whitehead tower of $B O(n)$ precisely implies $k$-orientability.

In a similar way as one could consider lifts to a sequence of connected covers of $B O(n)$ to give a homotopy-theoretic definition of $k$-parallisability, one can build a sequence of covers of $B O(n)$ such that lifts give rise to a $k$-orientation:

$$
B O(n) \leftarrow B S O(n) \leftarrow B \operatorname{Spin}(n) \leftarrow B O \mathrm{Br}_{3}(n) \leftarrow B O r_{4}(n) \leftarrow \ldots
$$


Here we formally define $B O r_{k}(n)$ up to homotopy by taking the homotopy fibre of the maps that classify the appropriate Stiefel-Whitney classes:

$$
\operatorname{BOr}_{k}(n) \rightarrow \operatorname{BOr}_{k-1}(n) \stackrel{w_{2^{k-1}}}{\longrightarrow} K\left(\mathbb{Z} / 2,2^{k-1}\right) .
$$

The class $w_{2^{k-1}}$ is non-trivial in the cohomology of $B O r_{k-1}=\lim _{n} B O r_{k-1}(n)$ by the lemma below.

Lemma 1.13. $w_{2^{k-1}}$ is a non-zero characteristic class of the map $B O r_{k-1} \rightarrow$ $B O$.

Proof. For $n$ such that $\phi(n)=k-1$ and $B O[n]$ the $n$-connected cover of $B O$, consider the diagram

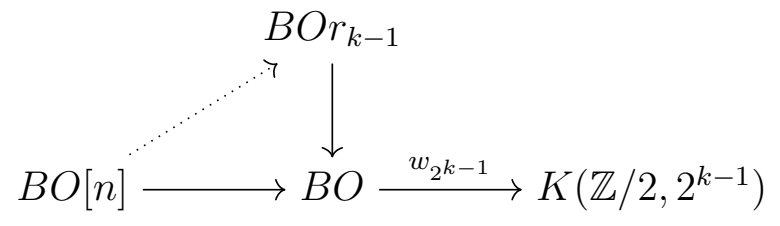

By the work of Stong in [Sto63], $B O[n]$ has the propery that its Stiefel-Whitney classes $w_{i}$ vanish for $0<i<2^{k-1}$, but $w_{2^{k-1}}$ does not vanish. Hence the map $B O[n] \rightarrow B O$ admits a lift to $B O r_{k-1}$, while the composite of the maps along the bottom of the diagram is not null-homotopic. Therefore the composite $\mathrm{BOr}_{k-1} \rightarrow \mathrm{BO} \stackrel{w_{2^{k-1}}}{\longrightarrow} K\left(\mathbb{Z} / 2,2^{k-1}\right)$ is not null-homotopic either.

By Lemma 1.8 it suffices to kill only the Stiefel-Whitney classes of the form $w_{2^{i}}$ for $i<k$ to classify $k$-orientable smooth manifolds.

For $k \geq 3$, the long exact sequence in homotopy corresponding to 1.22 reduces to the short exact sequence:

$$
0 \rightarrow \pi_{2^{k-1}}\left(B O r_{k}\right) \rightarrow \mathbb{Z} \stackrel{w_{2^{k-1}}}{\longrightarrow} \mathbb{Z} / 2 \rightarrow 0
$$


hence $\pi_{2^{k-1}}\left(B O r_{k}\right)=\mathbb{Z}$.

\subsection{Proof of Theorem 1.2}

We will now prove our main result that $k$-orientable manifolds have an even Euler characteristic as long as their dimension is not a multiple of $2^{k+1}$. In order to do so, we first prove a technical result that describes a relation in the Steenrod Algebra, arising from repeated application of the Adem relations. It tells us how Steenrod squares decompose that are $k-1$ times even but not $k$ times even.

Proposition 1.14. Let $n=2^{k} m+2^{k-1}$ for some $m \geq 0, k \geq 1$. Then

$$
S q^{n}=\sum_{i=1}^{2^{k-1}} S q^{i} \alpha^{n-i}
$$

where $\alpha^{n-i}$ is a sum of composites of squares.

Proof. For $m=0, n=2^{k-1}$, thus the result follows.

Assume $m \geq 1$. For $a$ and $b$ with $a<2 b$, the Adem relations can be rearranged as follows:

$$
\left(\begin{array}{c}
b-1 \\
a
\end{array}\right) S q^{a+b}=S q^{a} S q^{b}+\sum_{c=1}^{\lfloor a / 2\rfloor}\left(\begin{array}{c}
b-c-1 \\
a-2 c
\end{array}\right) S q^{a+b-c} S q^{c}
$$

Applying this to $a=2^{k-1}, b=2^{k} m$, with $n=a+b$, gives:

$$
\left(\begin{array}{c}
2^{k} m-1 \\
2^{k-1}
\end{array}\right) S q^{n}=S q^{2^{k-1}} S q^{2^{k} m}+\sum_{c=1}^{2^{k-2}}\left(\begin{array}{c}
2^{k} m-c-1 \\
2^{k-1}-2 c
\end{array}\right) S q^{n-c} S q^{c}
$$


By Lemma 1.10, the binomial $\left(\begin{array}{c}2^{k} m-1 \\ 2^{k-1}\end{array}\right)$ is non-zero. Hence we have now expressed $S q^{n}$ as the sum of a term with a small square in front, $S q^{2^{k-1}} S q^{2^{k} m}$, and a remainder. Each term in the remainder contains $S q^{n-c}$ for some $c$, with $1 \leq c \leq 2^{k-2}$. Define $i=2^{k-2}-c$ such that $S q^{n-c}=S q^{2^{k} m+i}$. We use strong induction on $i$ to show that each of these squares can be written in the desired form, as a sum of composites in which each term has a square on the left of degree less than or equal to $2^{k-1}$.

The base case is given by $i=1$. Applying equation 1.25 for $a=1, b=2^{k} m$ gives:

$$
\left(\begin{array}{c}
2^{k} m-1 \\
1
\end{array}\right) S q^{2^{k} m+1}=S q^{2^{k} m+1}=S q^{1} S q^{2^{k} m}
$$

which is of the desired form.

Suppose that $S q^{2^{k} m+i}$ can be written in the desired form for all $i<i_{0}$. We will show that $S q^{2^{k} m+i_{0}}$ can also be written in the desired form. Applying 1.25 for $a=i_{0}, b=2^{k} m$ gives:

$$
\begin{aligned}
\left(\begin{array}{c}
2^{k} m-1 \\
i_{0}
\end{array}\right) S q^{2^{k} m+i_{0}}=S q^{i_{0}} S q^{2^{k} m} & \\
& +\sum_{c=1}^{\left\lfloor i_{0} / 2\right\rfloor}\left(\begin{array}{c}
2^{k} m-c-1 \\
i_{0}-2 c
\end{array}\right) S q^{2^{k} m+i_{0}-c} S q^{c} .
\end{aligned}
$$

By Lemma 1.10, the binomial $\left(\begin{array}{c}2^{k} m-1 \\ i_{0}\end{array}\right)$ is non-zero. The first term on the right hand side is of the right form, and by the hypothesis, each $S q^{2^{k} m+i_{0}-c}$ can be written in the desired form. This concludes the induction.

Hence we observe that all terms in equation 1.26 are of the form $S q^{i} \alpha^{\prime n-i}$ for some composite of squares $\alpha^{\prime n-i}$. Collecting the terms with the same $S q^{i}$ in front and making use of the linearity of the squares renders the desired 
formula.

Theorem 1.15. Let $M^{n}$ be a manifold or Poincaré complex of dimension $n$. If $M$ is $k$-orientable for some $k$, then the $W u$ classes $v_{l}$ vanish for all $l$ such that $2^{k} \nmid l$.

Proof. By Lemma 1.11, $v_{i}=0$ for $0<i<2^{k}$ as a consequence of the $k$ orientability of $M$. Hence, by the definition of $v_{i}$, the operations

$$
S q^{i}: H^{n-i}(M) \rightarrow H^{n}(M)
$$

vanish for $i<2^{k}$.

Consider $v_{l}$ such that $2^{k} \nmid l$. Let $k^{\prime}-1<k$ be the highest power of 2 dividing l, such that $l=2^{k^{\prime}} m^{\prime}+2^{k^{\prime}-1}$ for some $m^{\prime}$. Then by Proposition 1.14 .

$$
S q^{l}=\sum_{i=1}^{2^{k^{\prime}-1}} S q^{i} \alpha^{l-i}
$$

for some operations $\alpha^{l-i}$. We consider this square with target the top dimension:

$$
S q^{l}: H^{n-l}(M ; \mathbb{Z} / 2) \rightarrow H^{n}(M ; \mathbb{Z} / 2)
$$

In the decomposition of $S q^{l}$, the operations

$$
S q^{i}: H^{n-i}(M ; \mathbb{Z} / 2) \rightarrow H^{n}(M ; \mathbb{Z} / 2)
$$

are of degree $0<i<2^{k^{\prime}-1}<2^{k}$ with target the top dimension and hence vanish. Therefore $S q^{l}$ with target the top dimension vanishes, which implies that $v_{l}=0$. 
Theorem 1.2 for $k \geq 1$ now follows as a corollary of the theorem above. Note that the case $k=0$ is a trivial consequence of Poincaré duality: odddimensional manifolds have zero, thus in particular even, Euler characteristic.

Corollary 1.16. A $k$-orientable manifold or Poincaré complex $M^{2 n}(k \geq 1)$ of dimension $2 n$ has an even Euler characteristic $\chi(M)$ if $2^{k+1} \nmid 2 n$.

Proof. We have that $2^{k} \nmid n$, hence the top Wu class $v_{n}$ vanishes by Theorem 1.3. By Theorem 1.7, this implies that the Euler characteristic is even. 


\section{Chapter 2}

\section{Orientability of the Rosenfeld planes}

\section{$2.1 \quad$ Introduction}

The fact that manifolds of odd dimension have a vanishing Euler characteristic is a straightforward consequence of Poincaré duality. For orientable manifolds, the Euler characteristic is moreover even in dimensions that are not a multiple of 4. Ochanine's theorem for the divisibility of the signature of spin manifolds implies that the Euler characteristic of spin manifolds is even unless the dimension is a multiple of 8 ([0ch81] $)$. This sequence of statements was generalised in the previous chapter: for higher orientable manifolds, the Euler characteristic is even in more and more dimensions (Theorem 1.2).

This chapter discusses the question of whether this theorem is strict: does a $k$-orientable manifold with odd Euler characteristic $(\chi)$ exist in all dimensions admitted by Theorem 1.2 , for all values of $k$ ? For $k=0,1,2,3$, the answer is 
yes, and the exemplifying manifolds are summarised in the following table.

\begin{tabular}{|c|l|c|c|}
\hline$k$ & corresponds to & $\begin{array}{c}\text { dimensions with } \\
\text { odd } \chi \text { possible }\end{array}$ & $\begin{array}{c}k \text {-orientable } \\
\text { manifolds with } \\
\text { odd } \chi\end{array}$ \\
\hline 0 & any manifold & $2 m$ & $\mathbb{R} P^{2 m}$ \\
1 & orientable manifolds & $4 m$ & $\mathbb{C P}^{2 m}$ \\
2 & spinable manifolds & $8 m$ & $\mathbb{H P}^{2 m}$ \\
3 & implied by stringable & $16 m$ & $\left(\mathbb{O P}^{2}\right)^{m}$ \\
4 & implied by fivebraneable & $32 m$ & $?$ \\
\hline
\end{tabular}

Whether a 4-orientable manifold with odd Euler characteristic exists is currently an open question. By theorem 1.2 , such manifolds would need to be of dimensions that are a multiple of 32. One might look for examples of highly orientable manifolds with odd Euler characteristic by considering special classes of manifolds that generalise the sequence of projective planes over division algebras. For example, [KS17] considers the existence of rational projective planes in different dimensions. Rational projective planes are simply connected, smooth, closed manifolds with rational cohomology isomorphic to $\mathbb{Q}[a] /\left\langle a^{3}\right\rangle$, and thus odd Euler characteristic. Kennard and Su show that these manifolds exist in dimensions 4, 8, 16, 32, 128 and 256, but there are none in any dimension between 256 and $2^{13}$, with the possible exception of 544 , 1024, 2048, 4160 and 4352. Whether there exist infinitely many rational projective planes remains an open question. However, the authors did show that no spinable rational projective planes exist in dimensions other than 8 and 16 ([KS17], Theorem C). Hence all rational projective planes besides those in the same dimensions as $\mathbb{H P}^{2}$ and $\mathbb{O P}^{2}$ are 1-orientable but not 2-orientable. 
A different direction in which to generalise the notion of projective planes is given by considering symmetric spaces. All projective spaces over different division algebras are in particular symmetric spaces of different Lie groups. Irreducible compact symmetric spaces were classified by Cartan and there are seven infinite families and twelve exceptional spaces in this classification. Recently, a uniform construction of all compact symmetric spaces was found, describing them as different types of Grassmannians using the four division algebras and the Freudenthal magic square ([HL11]). Three particularly interesting symmetric spaces in this context are given by the Rosenfeld planes of dimensions 32, 64 and 128. These manifolds have an odd Euler characteristic. They can be described as Lie group quotients of the exceptional groups $E_{6}$, $E_{7}$ and $E_{8}$ in the following way ([Bae02]):

$$
\begin{aligned}
& (\mathbb{O} \otimes \mathbb{C}) \mathbb{P}^{2}=E_{6} /((\operatorname{Spin}(10) \times U(1)) / \mathbb{Z} / 4) \\
& (\mathbb{O} \otimes \mathbb{H}) \mathbb{P}^{2}=E_{7} /((\operatorname{Spin}(12) \times S p(1)) / \mathbb{Z} / 2) \\
& (\mathbb{O} \otimes \mathbb{O}) \mathbb{P}^{2}=E_{8} /(\operatorname{Spin}(16) / \mathbb{Z} / 2)
\end{aligned}
$$

In Cartan's classification scheme the Rosenfeld planes are denoted by EIII, EVI and EVIII respectively. The cohomology of EIII or $(\mathbb{O} \otimes \mathbb{C}) \mathbb{P}^{2}$ is known integrally, for EVI or $(\mathbb{O} \otimes \mathbb{H}) \mathbb{P}^{2}$ the cohomology is known modulo 2 and for EVIII or $(\mathbb{O} \otimes \mathbb{O}) \mathbb{P}^{2}$, as well as EV and EIX in the classification scheme, the cohomology remains undetermined.

Rather than requiring the lowest Stiefel-Whitney classes to vanish, one can equivalently require that $\mathrm{Wu}$ classes in the same degrees vanish (Lemma 1.11). Hence, whether a manifold is $k$-orientable can be established by considering the 
action of the Steenrod algebra on its cohomology. In this chapter we consider the orientability of $(\mathbb{O} \otimes \mathbb{C}) \mathbb{P}^{2}$ and $(\mathbb{O} \otimes \mathbb{H}) \mathbb{P}^{2}$ by considering the action of the Steenrod algebra on their mod 2 cohomology. The integral cohomology of $(\mathbb{O} \otimes$ C) $\mathbb{P}^{2}$ was determined in [TW74], and the action of the Steenrod algebra was determined in Ish92. The mod 2 cohomology of $(\mathbb{O} \otimes \mathbb{H}) \mathbb{P}^{2}$ was determined in [Nak01], and in the process the action of the Steenrod algebra was established partially. We here calculate the action of specific Steenrod squares with target the top cohomology in order to further our understanding of the orientability of $(\mathbb{O} \otimes \mathbb{C}) \mathbb{P}^{2}$ and $(\mathbb{O} \otimes \mathbb{H}) \mathbb{P}^{2}$. The computations make use of a program written in Mathematica by the author that aids in the expansion of the Cartan formula on large products. This leads to the following results for the orientability of $(\mathbb{O} \otimes \mathbb{C}) \mathbb{P}^{2}$ and $(\mathbb{O} \otimes \mathbb{H}) \mathbb{P}^{2}$

Theorem 2.1 (Theorem 2.6). $(\mathbb{O} \otimes \mathbb{C}) \mathbb{P}^{2}$ is 2-orientable and not 3-orientable.

Theorem 2.2. $(\mathbb{O} \otimes \mathbb{H}) \mathbb{P}^{2}$ is 3-orientable. It is 4-orientable if $\beta^{\prime \prime}+\nu^{\prime \prime}=1$, where $\beta^{\prime \prime}$ is the prefactor of $y_{2} y_{3}^{2} y_{12}$ in $S q^{8} y_{12}$ and $\nu^{\prime \prime}$ is the prefactor of $y_{2} y_{3}^{2} y_{20}$ in $S q^{8} y_{20}$.

The prefactors $\beta^{\prime \prime}$ and $\nu^{\prime \prime}$ are part of the indeterminacy in our current knowledge of the action of the Steenrod algebra on the mod 2 cohomology of $(\mathbb{O} \otimes \mathbb{H}) \mathbb{P}^{2}$ as established in [Nak01].

\subsection{Orientability of $E I I I$ or $(\mathbb{O} \otimes \mathbb{C}) \mathbb{P}^{2}$}

In this section we determine that the first Rosenfeld plane $(\mathbb{O} \otimes \mathbb{C}) \mathbb{P}^{2}$ is 2 orientable and not 3-orientable by performing calculations of the action of the 
Steenrod algebra on its cohomology. The integral cohomology of this manifold was determined as a ring in [TW74]:

$$
H^{*}\left((\mathbb{O} \otimes \mathbb{C}) \mathbb{P}^{2} ; \mathbb{Z}\right)=\mathbb{Z}[t, w] /\left(t^{9}-3 w^{2} t, w^{3}+15 w^{2} t^{4}-9 w t^{8}\right)
$$

where $t$ is in degree 2 and $w$ in degree 8. From this expression we determined an additive basis in every degree using Mathematica, the result of which can be found on $\left[\right.$ Hoe] under Additive cohomology of $(\mathbb{O} \otimes \mathbb{C}) \mathbb{P}^{2}$. As the integral cohomology is concentrated in even degrees, the mod 2 cohomology is given by

$$
H^{*}\left((\mathbb{O} \otimes \mathbb{C}) \mathbb{P}^{2} ; \mathbb{Z} / 2\right)=H^{*}\left((\mathbb{O} \otimes \mathbb{C}) \mathbb{P}^{2} ; \mathbb{Z}\right) \otimes \mathbb{Z} / 2
$$

We write $t^{\prime}$ and $w^{\prime}$ for the mod 2 reductions of the generators $t$ and $w$. The calculations are done by considering a general expression for the Steenrod operations, for which we subsequently substitute the coefficients as established in Ish92.

Lemma 2.3. The action of the Steenrod algebra on $H^{*}\left((\mathbb{O} \otimes \mathbb{C}) \mathbb{P}^{2} ; \mathbb{Z} / 2\right)$ is fully determined by four coefficients $\alpha, \beta, \gamma, \delta \in \mathbb{Z} / 2$, where:

$$
\begin{aligned}
& S q^{2} w^{\prime}=\alpha t^{\prime 5}+\beta t^{\prime} w^{\prime} \\
& S q^{4} w^{\prime}=\gamma t^{\prime 6}+\delta t^{\prime 2} w^{\prime}
\end{aligned}
$$

Proof of Lemma 2.3. As there is no odd degree cohomology, all odd squares are zero. The action of $S q^{i}$ on any class can be decomposed into its action on $t^{\prime}$ and $w^{\prime}$ by use of the Cartan formula. Hence the entire action of the Steenrod algebra is determined by $S q^{2} t^{\prime}=t^{\prime 2}, S q^{2} w^{\prime}, S q^{4} w^{\prime}$ and $S q^{8} w^{\prime}=w^{\prime 2}$. 
$H^{10}\left((\mathbb{O} \otimes \mathbb{C}) \mathbb{P}^{2} ; \mathbb{Z} / 2\right)$ is spanned by $t^{\prime 5}$ and $t^{\prime} w^{\prime}$, therefore $S q^{2} w^{\prime}$ is some linear combination of these. Similarly $H^{12}\left((\mathbb{O} \otimes \mathbb{C}) \mathbb{P}^{2} ; \mathbb{Z} / 2\right)$ is spanned by $t^{\prime 6}$ and $t^{\prime 2} w^{\prime}$

We list the signature of the manifold, which follows from the explicit computations.

Lemma 2.4. The signature of $(\mathbb{O} \otimes \mathbb{C}) \mathbb{P}^{2}$ is 3 .

Proof of Theorem 2.4. Computer calculations show that $H^{32}\left((\mathbb{O} \otimes \mathbb{C}) \mathbb{P}^{2} ; \mathbb{Z}\right) \cong$ $\mathbb{Z}$ with $t^{16}=78, t^{12} w=45, t^{8} w^{2}=26, t^{4} w^{3}=15$ and $w^{4}=9 . \quad H^{16}((\mathbb{O} \otimes$ $\left.\mathbb{C}) \mathbb{P}^{2} ; \mathbb{Z}\right) \cong \mathbb{Z}^{3}$ spanned by $t^{8}, t^{4} w$ and $w^{2}$ hence in this basis the cup square is given by

$$
\left(\begin{array}{ccc}
78 & 45 & 26 \\
45 & 26 & 15 \\
26 & 15 & 9
\end{array}\right)
$$

which has three positive eigenvalues.

The orientability of $(\mathbb{O} \otimes \mathbb{C}) \mathbb{P}^{2}$ can be established by computing the action of the Steenrod squares reaching up to the top dimension.

Proposition 2.5. For $\alpha, \beta, \gamma, \delta \in \mathbb{Z} / 2$ as in Lemma 2.3, $(\mathbb{O} \otimes \mathbb{C}) \mathbb{P}^{2}$ is 2orientable if $\beta=1$; 3-orientable if also $\delta=1$, in which case it is also 4orientable.

Proof. As $H^{1}\left((\mathbb{O} \otimes \mathbb{C}) \mathbb{P}^{2} ; \mathbb{Z}\right)=0$, we have $w_{1}=0$ hence $(\mathbb{O} \otimes \mathbb{C}) \mathbb{P}^{2}$ is $(1$ )orientable.

Computer calculations show that $H^{30}\left((\mathbb{O} \otimes \mathbb{C}) \mathbb{P}^{2} ; \mathbb{Z}\right) \cong \mathbb{Z}$ with $t^{15}=78$, $t^{11} w=45, t^{7} w^{2}=26$ and $t^{3} w^{3}=15$. In order to check whether $w_{2}=0$, 
we check whether $S q^{2}: H^{30}\left((\mathbb{O} \otimes \mathbb{C}) \mathbb{P}^{2} ; \mathbb{Z} / 2\right) \rightarrow H^{32}\left((\mathbb{O} \otimes \mathbb{C}) \mathbb{P}^{2} ; \mathbb{Z} / 2\right)$ vanishes. Denote by $t^{\prime}$ and $w^{\prime}$ the images of $t$ and $w$ under the ring homomorphism given by tensoring $H^{*}\left((\mathbb{O} \otimes \mathbb{C}) \mathbb{P}^{2} ; \mathbb{Z}\right)$ with $\mathbb{Z} / 2$. Then the generator of $H^{30}\left((\mathbb{O} \otimes \mathbb{C}) \mathbb{P}^{2} ; \mathbb{Z} / 2\right)$ can be represented as $t^{\prime 11} w^{\prime}$ or as $t^{\prime 3} w^{\prime 3}$. Applying $S q^{2}$ to this and using the Cartan formula:

$$
\begin{aligned}
S q^{2}\left(t^{\prime 11} w^{\prime}\right) & =S q^{2}\left(t^{\prime 11}\right) w^{\prime}+t^{\prime 11} S q^{2}\left(w^{\prime}\right) \\
& =t^{\prime 12} w^{\prime}+t^{\prime 11}\left(\alpha t^{\prime 5}+\beta t^{\prime} w^{\prime}\right) \\
& =(1+\beta) t^{\prime 12} w^{\prime}+\alpha t^{\prime 16} \\
& =(1+\beta) t^{\prime 12} w^{\prime} \\
& =(1+\beta)
\end{aligned}
$$

And similarly

$$
\begin{aligned}
S q^{2}\left(t^{\prime 3} w^{\prime 3}\right) & =(1+\beta) t^{\prime 4} w^{\prime 3} \\
& =(1+\beta)
\end{aligned}
$$

Both $t^{\prime 2} w^{\prime}$ and $t^{\prime 4} w^{\prime 3}$ represent the generator of $H^{32}\left((\mathbb{O} \otimes \mathbb{C}) \mathbb{P}^{2} ; \mathbb{Z} / 2\right)$, hence $w_{2}=0$ if and only if $\beta=1$.

$H^{28}\left((\mathbb{O} \otimes \mathbb{C}) \mathbb{P}^{2} ; \mathbb{Z}\right) \cong \mathbb{Z}$ with $t^{14}=78, t^{10} w=45, t^{6} w^{2}=26$ and $t^{2} w^{3}=15$ hence $H^{28}\left((\mathbb{O} \otimes \mathbb{C}) \mathbb{P}^{2} ; \mathbb{Z} / 2\right) \cong \mathbb{Z} / 2$ generated by $t^{\prime 10} w^{\prime}$ or equivalently $t^{\prime 2} w^{\prime 3}$. 


$$
\begin{aligned}
S q^{4}\left(t^{\prime 10} w^{\prime}\right) & =S q^{4}\left(t^{\prime 10}\right) w^{\prime}+S q^{2}\left(t^{\prime 10}\right) S q^{2}\left(w^{\prime}\right)+t^{\prime 10} S q^{4}\left(w^{\prime}\right) \\
& =\left(\begin{array}{c}
10 \\
2
\end{array}\right)\left(S q^{2}\left(t^{\prime}\right)\right)^{2} t^{\prime 8} w^{\prime}+0+t^{\prime 10}\left(\gamma t^{\prime 6}+\delta t^{\prime 2} w^{\prime}\right) \\
& =(1+\delta) t^{\prime 12} w^{\prime}+\gamma t^{\prime 16} \\
& =(1+\delta) t^{\prime 12} w^{\prime} \\
& =(1+\delta)
\end{aligned}
$$

and similarly

$$
\begin{aligned}
S q^{4}\left(t^{\prime 2} w^{\prime 3}\right) & =(1+\delta) t^{\prime 4} w^{\prime 3} \\
& =(1+\delta)
\end{aligned}
$$

Hence $(\mathbb{O} \otimes \mathbb{C}) \mathbb{P}^{2}$ is 3-orientable if and only if $\beta=1$ and $\delta=1$.

$H^{24}\left((\mathbb{O} \otimes \mathbb{C}) \mathbb{P}^{2} ; \mathbb{Z}\right)=\cong \mathbb{Z}^{2}$ with $t^{12}=(3,9), t^{18} w=(2,5), t^{4} w^{2}=(1,3)$ and $w^{3}=(3,0)$. After tensoring with $\mathbb{Z} / 2$, we thus get that in $H^{24}\left((\mathbb{O} \otimes \mathbb{C}) \mathbb{P}^{2} ; \mathbb{Z} / 2\right)$, $t^{\prime 12}=(1,1), t^{\prime 8} w^{\prime}=(0,1), t^{\prime 4} w^{\prime 2}=(1,1)$ and $w^{\prime 3}=(1,0)$. First we act on $t^{\prime 8} w^{\prime}=(0,1)$.

$$
\begin{aligned}
S q^{8}\left(t^{\prime 8} w^{\prime}\right)= & S q^{8}\left(t^{\prime 8}\right) w^{\prime}+S q^{6}\left(t^{\prime 8}\right) S q^{2}\left(w^{\prime}\right)+S q^{4}\left(t^{\prime 8}\right) S q^{4}\left(w^{\prime}\right) \\
& +S q^{2}\left(t^{\prime 8}\right) S q^{6}\left(w^{\prime}\right)+t^{\prime 8} S q^{8}\left(w^{\prime}\right) \\
= & t^{\prime 8} w^{\prime 2} \\
= & 0 .
\end{aligned}
$$


Alternatively, acting on $t^{\prime 12}=(1,1)$ gives

$$
\begin{aligned}
S q^{8}\left(t^{\prime 2}\right) & =t^{8}\left(S q^{2} t^{\prime}\right)^{4} \quad \text { (Using Mathematica) } \\
& =t^{16} \\
& =0
\end{aligned}
$$

hence $S q^{8}: H^{24}\left((\mathbb{O} \otimes \mathbb{C}) \mathbb{P}^{2} ; \mathbb{Z} / 2\right) \rightarrow H^{32}\left((\mathbb{O} \otimes \mathbb{C}) \mathbb{P}^{2} ; \mathbb{Z} / 2\right)$ also vanishes on $(1,0)=(1,1)+(0,1)$, thus it vanishes entirely. This implies that $(\mathbb{O} \otimes \mathbb{C}) \mathbb{P}^{2}$ is automatically 4 -orientable if it is 3 -orientable.

Theorem 2.6. $(\mathbb{O} \otimes \mathbb{C}) \mathbb{P}^{2}$ is 2-orientable (spin) and not 3-orientable.

Proof. By [Ish92], $\alpha=\beta=\gamma=1$ and $\delta=0$. Hence by Proposition 2.5. $(\mathbb{O} \otimes \mathbb{C}) \mathbb{P}^{2}$ is 2 -orientable but not 3 -orientable.

\subsection{Orientability of $E V I$ or $(\mathbb{O} \otimes \mathbb{H}) \mathbb{P}^{2}$}

For $(\mathbb{O} \otimes \mathbb{H}) \mathbb{P}^{2}$ the $\bmod 2$ cohomology was established as a ring by [Nak01, and it is generated by five classes in degrees 2, 3, 12, 16 and 20, modulo an ideal generated by twelve relations. In [Nak01] also the action of $S q^{1}, S q^{2}$, $S q^{4}$ and $S q^{8}$ was determined on all generators, modulo twelve undetermined coefficients. We here perform calculations that show that $(\mathbb{O} \otimes \mathbb{H}) \mathbb{P}^{2}$ is at least 3-orientable, but because of two of the undetermined coefficients it cannot be established from this information whether $(\mathbb{O} \otimes \mathbb{H}) \mathbb{P}^{2}$ is 4-orientable.

Theorem 2.7 ([Nak01]).

$$
H^{*}\left((\mathbb{O} \otimes \mathbb{H}) \mathbb{P}^{2}, \mathbb{Z} / 2\right)=\mathbb{Z} / 2\left[y_{2}, y_{3}, y_{12}, y_{16}, y_{20}\right] / J
$$


where $J$ is an ideal generated by twelve homogeneous relations:

$$
J=\left(\begin{array}{l}
y_{3}^{3}, y_{16} y_{2}+y_{12} y_{3}^{2}+y_{2}^{6} y_{3}^{2}, y_{16} y_{3}, y_{12}^{2} y_{2}+y_{12} y_{2}^{4} y_{3}^{2}+y_{20} y_{3}^{2}, \\
y_{12}^{2} y_{3}, y_{12} y_{16}+y_{2}^{14}+y_{12} y_{2}^{5} y_{3}^{2}+y_{2}^{11} y_{3}^{2}, y_{12}^{3}+y_{16} y_{20}+y_{2}^{5} y_{20} y_{3}^{2}, \\
y_{12}^{2} y_{16}+y_{20}^{2}+y_{12} y_{2}^{11} y_{3}^{2}, y_{12}^{2} y_{20}+y_{12} y_{2}^{13} y_{3}^{2}+y_{12} y_{2}^{3} y_{20} y_{3}^{2} \\
y_{12} y_{16}^{2}+y_{12} y_{2}^{13} y_{3}^{2}, y_{16}^{3}+y_{12} y_{16} y_{20}+y_{12} y_{2}^{5} y_{20} y_{3}^{2}, y_{16}^{2} y_{20}+y_{2}^{13} y_{20} y_{3}^{2}
\end{array}\right)
$$

Along the way to establishing Theorem 2.7, a number of Steenrod operations are determined in [Nak01] and used in the computation.

Lemma 2.8 ([Nak01]). S $S q^{1} y_{i}=0$ unless $i=2$, in which case

$$
S q^{1} y_{2}=y_{3}
$$

Lemma 2.9 ([Nak01]).

$$
\begin{aligned}
& S q^{2}\left(y_{12}\right)=y_{2}^{7}+y_{2} y_{12}+y_{2}^{4} y_{3}^{2}, \\
& S q^{4}\left(y_{12}\right)=y_{2}^{8}+y_{2}^{2} y_{12}+\alpha^{\prime} y_{2}^{5} y_{3}^{2}, \\
& S q^{8}\left(y_{12}\right)=y_{20}+y_{2}^{4} y_{12}+\alpha^{\prime \prime} y_{2}^{7} y_{3}^{2}+\beta^{\prime \prime} y_{2} y_{3}^{2} y_{12},
\end{aligned}
$$

for some coefficients $\alpha^{\prime}, \alpha^{\prime \prime}, \beta^{\prime \prime} \in \mathbb{Z} / 2$. 
Lemma 2.10 ([Nak01]).

$$
\begin{aligned}
& S q^{2}\left(y_{16}\right)=0 \\
& S q^{4}\left(y_{16}\right)=y_{2}^{7} y_{3}^{2} \\
& S q^{8}\left(y_{16}\right)=y_{12}^{2}+\gamma^{\prime \prime} y_{2}^{9} y_{3}^{2}+\delta^{\prime \prime} y_{2}^{3} y_{3}^{2} y_{12},
\end{aligned}
$$

for some coefficients $\gamma^{\prime \prime}, \delta^{\prime \prime} \in \mathbb{Z} / 2$.

Lemma 2.11 ([Nak01]).

$$
\begin{aligned}
& S q^{2}\left(y_{20}\right)=y_{2}^{11}+y_{2} y_{20}+\mu y_{2}^{8} y_{3}^{2}+\nu y_{2}^{2} y_{3}^{2} y_{12}, \\
& S q^{4}\left(y_{20}\right)=y_{12}^{2}+y_{2}^{6} y_{12}+\mu^{\prime} y_{2}^{9} y_{3}^{2}+\nu^{\prime} y_{2}^{3} y_{3}^{2} y_{12}, \\
& S q^{8}\left(y_{20}\right)=y_{12} y_{16}+y_{2}^{8} y_{12}+\lambda^{\prime \prime} y_{2}^{11} y_{3}^{2}+\mu^{\prime \prime} y_{2}^{5} y_{3}^{2} y_{12}+\nu^{\prime \prime} y_{2} y_{3}^{2} y_{20},
\end{aligned}
$$

for some coefficients $\mu, \nu, \mu^{\prime}, \nu^{\prime}, \lambda^{\prime \prime}, \mu^{\prime \prime}, \nu^{\prime \prime} \in \mathbb{Z} / 2$.

In order to determine the entire action of the Steenrod algebra on the cohomology of $(\mathbb{O} \otimes \mathbb{H}) \mathbb{P}^{2}$, one would need to establish the values of the twelve unknown coefficients above and compute $S q^{16} y_{20}$.

In the sections below we calculate the remaining actions of the Steenrod operations up to $S q^{8}$ on the generators, namely the action of $S q^{3}, S q^{5}, S q^{6}$ and $S q^{7}$. A program in Mathematica was written to aid with the expansion of the Cartan formula on larger products and to automatically replace the previously established smaller squaring operations, which can be found on [Hoe] under Expanding the Cartan formula in Mathematica. 
After establishing these smaller squares we calculate the action of $S q^{1}, S q^{2}$, $S q^{4}$ and $S q^{8}$ up to the top dimension to establish the orientability of $(\mathbb{O} \otimes \mathbb{H}) \mathbb{P}^{2}$. The use of the Mathematica code is essential especially for these latter calculations as the expansion of the Cartan formula on larger products with high squares involves a large number of terms. For example, the Cartan formula for expanding $S q^{8}$ on a product of twelve generators contains 75,582 terms, each of which is a product of twelve sums with up to five terms each, most of which are repeated an even number of times and hence vanish in the end. A note is made in all places where Mathematica was used. 


\subsubsection{Computing the action of $S q^{3}$ on the generators}

$$
\begin{aligned}
& S q^{3} y_{3}=y_{3}^{2} \\
& S q^{3} y_{12}=S q^{1} S q^{2} y_{12} \\
& =S q^{1}\left(y_{2}^{7}+y_{2} y_{12}+y_{2}^{4} y_{3}^{2}\right) \\
& =S q^{1}\left(y_{2}^{7}\right)+S q^{1}\left(y_{2} y_{12}\right)+S q^{1}\left(y_{2}^{4} y_{3}^{2}\right) \\
& =y_{2}^{6} y_{3}+y_{3} y_{12}+0+S q^{1}\left(y_{2}^{4}\right) y_{3}^{2}+y_{2}^{4} S q^{1}\left(y_{3}^{2}\right) \\
& =y_{2}^{6} y_{3}+y_{3} y_{12} \\
& S q^{3} y_{16}=S q^{1} S q^{2} y_{16} \\
& =0 \\
& S q^{3} y_{20}=S q^{1} S q^{2} y_{20} \\
& =S q^{1}\left(y_{2}^{11}+y_{2} y_{20}+\mu y_{2}^{8} y_{3}^{2}+\nu y_{2}^{2} y_{3}^{2} y_{12}\right) \\
& =S q^{1}\left(y_{2}\right) y_{2}^{10}+S q^{1}\left(y_{2}\right) y_{20}+y_{2} S q^{1}\left(y_{20}\right)+\mu S q^{1}\left(y_{2}^{8} y_{3}^{2}\right)+\nu S q^{1}\left(y_{2}^{2} y_{3}^{2} y_{12}\right) \\
& =y_{2}^{10} y_{3}+y_{3} y_{20}+\mu S q^{1}\left(y_{2}^{8}\right) y_{3}^{2}+\nu S q^{1}\left(y_{2}^{2}\right) y_{3}^{2} y_{12} \\
& =y_{2}^{10} y_{3}+y_{3} y_{20}
\end{aligned}
$$




\subsubsection{Computing the action of $S q^{5}$ on the generators}

$$
\begin{aligned}
S q^{5} y_{12} & =S q^{1} S q^{4} y_{12} \\
& =S q^{1}\left(y_{2}^{8}+y_{2}^{2} y_{12}+\alpha^{\prime} y_{2}^{5} y_{3}^{2}\right) \\
& =\alpha^{\prime} y_{2}^{4} y_{3}^{3}
\end{aligned}
$$

$$
\begin{aligned}
S q^{5} y_{16} & =S q^{1} S q^{4} y_{16} \\
& =S q^{1}\left(y_{2}^{7} y_{3}^{2}\right) \\
& =y_{2}^{6} y_{3}^{3}
\end{aligned}
$$

$$
\begin{aligned}
S q^{5} y_{20}= & S q^{1} S q^{4} y_{20} \\
= & S q^{1}\left(y_{12}^{2}+y_{2}^{6} y_{12}+\mu^{\prime} y_{2}^{9} y_{3}^{2}+\nu^{\prime} y_{2}^{3} y_{3}^{2} y_{12}\right) \\
= & S q^{1}\left(y_{12}^{2}\right)+S q^{1}\left(y_{2}^{6} y_{12}\right)+\mu^{\prime} S q^{1}\left(y_{2}^{9} y_{3}^{2}\right)+\nu^{\prime} S q^{1}\left(y_{2}^{3} y_{3}^{2} y_{12}\right) \\
= & S q^{1}\left(y_{2}^{6}\right) y_{12}+y_{2}^{6} S q^{1}\left(y_{12}\right)+\mu^{\prime} S q^{1}\left(y_{2}^{9}\right) y_{3}^{2}+\mu^{\prime} y_{2}^{9} S q^{1}\left(y_{3}^{2}\right) \\
& +\nu^{\prime} S q^{1}\left(y_{2}^{3}\right) y_{3}^{2} y_{12}+\nu^{\prime} y_{2}^{3} S q^{1}\left(y_{3}^{2}\right) y_{12}+\nu^{\prime} y_{2}^{3} y_{3}^{2} S q^{1}\left(y_{12}\right) \\
= & 0+0+\mu^{\prime} S q^{1}\left(y_{2}^{9}\right) y_{3}^{2}+0+\nu^{\prime} S q^{1}\left(y_{2}^{3}\right) y_{3}^{2} y_{12}+0+0 \\
= & \mu^{\prime} y_{2}^{8} y_{3}^{3}+\nu^{\prime} y_{2}^{2} y_{3}^{3} y_{12}
\end{aligned}
$$




\subsubsection{Computing the action of $S q^{6}$ on the generators}

$$
\begin{aligned}
S q^{6} y_{12}= & S q^{5} S q^{1} y_{12}+S q^{2} S q^{4} y_{12} \\
= & 0+S q^{2}\left(y_{2}^{8}+y_{2}^{2} y_{12}+\alpha^{\prime} y_{2}^{5} y_{3}^{2}\right) \\
= & \left(S q^{1}\left(y_{2}^{4}\right)\right)^{2}+S q^{2}\left(y_{2}^{2} y_{12}\right)+\alpha^{\prime} S q^{2}\left(y_{2}^{5} y_{3}^{2}\right) \\
= & 0+y_{2}^{9}+y_{2}^{6} y_{3}^{2}+y_{2}^{3} y_{12}+y_{3}^{2} y_{12}+\alpha^{\prime} y_{2}^{6} y_{3}^{2} \quad \text { (Using Mathematica) } \\
= & y_{2}^{9}+\left(1+\alpha^{\prime}\right) y_{2}^{6} y_{3}^{2}+y_{2}^{3} y_{12}+y_{3}^{2} y_{12} \\
S q^{6} y_{16}= & S q^{5} S q^{1} y_{16}+S q^{2} S q^{4} y_{16} \\
= & 0+S q^{2}\left(y_{2}^{7} y_{3}^{2}\right) \\
= & S q^{2}\left(y_{2}^{7}\right) y_{3}^{2}+S q^{1}\left(y_{2}^{7}\right) S q^{1}\left(y_{3}^{2}\right)+y_{2}^{7} S q^{2}\left(y_{3}^{2}\right) \\
= & \left(y_{2}^{8}+y_{2}^{6} y_{3}\right) y_{3}^{2}+0+0 \\
= & y_{2}^{8} y_{3}^{2}+y_{2}^{6} y_{3}^{3} \\
& +\nu^{\prime}\left(y_{2}^{10} y_{3}^{2}+y_{2}^{7} y_{3}^{4}+y_{2} y_{3}^{4} y_{12}\right) \quad(\mathrm{U} \operatorname{sing} \text { Mathematica }) \\
= & y_{2}^{13}+\left(1+\mu^{\prime}+\nu^{\prime}\right) y_{2}^{10} y_{3}^{2}+y_{2}^{7} y_{12}+y_{2}^{4} y_{3}^{2} y_{12}+\nu^{\prime}\left(y_{2}^{7} y_{3}^{4}+y_{2} y_{3}^{4} y_{12}\right) \\
S q^{6} y_{20}= & S q^{5} S q^{1} y_{20}+S q^{2} S q^{4} y_{20} \\
= & 0+S q^{2}\left(y_{12}^{2}+y_{2}^{6} y_{12}+\mu^{\prime} y_{2}^{9} y_{3}^{2}+\nu^{\prime} y_{2}^{3} y_{3}^{2} y_{12}\right) \\
= & \left(S q^{1} y_{12}\right)^{2}+S q^{2}\left(y_{2}^{6} y_{12}\right)+\mu^{\prime} S q^{2}\left(y_{2}^{9} y_{3}^{2}\right)+\nu^{\prime} S q^{2}\left(y_{2}^{3} y_{3}^{2} y_{12}\right) \\
= & 0+y_{2}^{13}+y_{2}^{10} y_{3}^{2}+y_{2}^{7} y_{12}+y_{2}^{4} y_{3}^{2} y_{12}+\mu^{\prime}\left(y_{2}^{10} y_{3}^{2}\right) \\
= & \\
= &
\end{aligned}
$$




\subsubsection{Computing the action of $S q^{7}$ on the generators}

$$
\begin{aligned}
S q^{7} y_{12} & =S q^{1} S q^{6}\left(y_{12}\right) \\
& =S q^{1}\left(y_{2}^{9}+\left(1+\alpha^{\prime}\right) y_{2}^{6} y_{3}^{2}+y_{2}^{3} y_{12}+y_{3}^{2} y_{12}\right) \\
& =S q^{1}\left(y_{2}^{9}\right)+\left(1+\alpha^{\prime}\right) S q^{1}\left(y_{2}^{6} y_{3}^{2}\right)+S q^{1}\left(y_{2}^{3} y_{12}\right)+S q^{1}\left(y_{3}^{2} y_{12}\right) \\
& =y_{2}^{8} y_{3}+0+y_{2}^{2} y_{3} y_{12}+0 \\
& =y_{2}^{8} y_{3}+y_{2}^{2} y_{3} y_{12} \\
S q^{7} y_{16} & =S q^{1} S q^{6}\left(y_{16}\right) \\
& =S q^{1}\left(y_{2}^{8} y_{3}^{2}+y_{2}^{6} y_{3}^{3}\right) \\
& =S q^{1}\left(y_{2}^{8} y_{3}^{2}\right)+S q^{1}\left(y_{2}^{6} y_{3}^{3}\right) \\
& =0 \\
& =S q^{7} y_{20}=S q^{1} S q^{6}\left(y_{20}\right) \\
& =q^{1}\left(y_{2}^{13}+\left(1+\mu^{\prime}+\nu^{\prime}\right) y_{2}^{10} y_{3}^{2}+y_{2}^{7} y_{12}+y_{2}^{4} y_{3}^{2} y_{12}+\nu^{\prime} y_{2}^{7} y_{3}^{4}+\nu^{\prime} y_{2} y_{3}^{4} y_{12}\right) \\
& =y_{2}^{12} y_{3}+0+y_{2}^{6} y_{3} y_{12}+0+\nu_{2}^{6} y_{3} y_{12} y_{3}^{5}+\nu^{\prime} y_{3}^{5} y_{12} y_{2}^{6} y_{3}^{5}+\nu^{\prime} y_{3}^{5} y_{12} \\
& \\
&
\end{aligned}
$$

\subsubsection{3-orientability of $(\mathbb{O} \otimes \mathbb{H}) \mathbb{P}^{2}$}

The orientability of $(\mathbb{O} \otimes \mathbb{H}) \mathbb{P}^{2}$ can be established by calculating the actions of the Steenrod squares that reach up to the top dimension. An additive basis for the cohomology, together with the monomials in all generators expressed in this basis, was calculated from the presentation given in Theorem 2.7 using 
MAGMA, with the help of Robert Bruner. It can be found on Hoe under Monomials in additive cohomology of $(\mathbb{O} \otimes \mathbb{H}) \mathbb{P}^{2}$. This description lists all monomials in the five generators $y_{2}, y_{3}, y_{12}, y_{16}$ and $y_{20}$ up to degree 64 , listing which of these are zero in the cohomology and which are equated. The list of ranks of $H^{*}\left((\mathbb{O} \otimes \mathbb{H}) \mathbb{P}^{2} ; \mathbb{Z} / 2\right)$ from dimension 0 up to and including dimension 32 is as follows:

$(1,0,1,1,1,1,2,1,2,1,2,1,3,1,3,2,4,2,4,2,5,2,5,3,6,3,6,3,6,3,6,5,2,7)$.

The image of $S q^{1}$ is also calculated in Nak01] and since $H^{*}\left((\mathbb{O} \otimes \mathbb{H}) \mathbb{P}^{2} ; \mathbb{Z}\right)$ has only 2-torsion, the integral cohomology of $(\mathbb{O} \otimes \mathbb{H}) \mathbb{P}^{2}$ can be determined additively from this information.

Lemma 2.12. $(\mathbb{O} \otimes \mathbb{H}) \mathbb{P}^{2}$ is 1-orientable.

Proof. $H^{1}\left((\mathbb{O} \otimes \mathbb{H}) \mathbb{P}^{2}, \mathbb{Z} / 2\right)=0$, hence $w_{1}=0$.

Theorem 2.13. $(\mathbb{O} \otimes \mathbb{H}) \mathbb{P}^{2}$ is 2-orientable.

Proof. In order to establish 2-orientability of $(\mathbb{O} \otimes \mathbb{H}) \mathbb{P}^{2}$, we will compute the action of $S q^{2}$ on $H^{62}\left((\mathbb{O} \otimes \mathbb{H}) \mathbb{P}^{2} ; \mathbb{Z} / 2\right)$, which is generated by $y_{2} y_{20}^{3}$. We expand the Cartan formula with the use of Mathematica, and substitute in the results for the smaller squares obtained in sections 2.3.1-2.3.4. This gives:

$$
\begin{aligned}
S q^{2}\left(y_{2} y_{20}^{3}\right) & =\sum_{i+j+k+l=2} S q^{i} y_{2} S q^{j} y_{20} S q^{k} y_{20} S q^{l} y_{20} \\
& =y_{2}^{12} y_{20}^{2}+\mu y_{2}^{9} y_{3}^{2} y_{20}^{2}+\nu y_{2}^{3} y_{3}^{2} y_{12} y_{20}^{2} \\
& =0
\end{aligned}
$$


Hence we see that the $S q^{2}$ to the top dimension vanishes, hence $w_{2}=0$. As $(\mathbb{O} \otimes \mathbb{H}) \mathbb{P}^{2}$ is a smooth manifold, $w_{3}=0$ as well.

Theorem 2.14. $(\mathbb{O} \otimes \mathbb{H}) \mathbb{P}^{2}$ is 3-orientable.

Proof. $H^{60}\left((\mathbb{O} \otimes \mathbb{H}) \mathbb{P}^{2} ; \mathbb{Z} / 2\right) \cong \mathbb{Z} / 2$ generated by $y_{20}^{3}$. Expanding the Cartan formula and substituting in lower squares in Mathematica we see that

$$
\begin{aligned}
S q^{4}\left(y_{20}^{3}\right)= & \sum_{i+j+k=4} S q^{i} y_{20} S q^{j} y_{20} S q^{k} y_{20} \\
= & y_{2}^{22} y_{20}+\mu^{2} y_{2}^{16} y_{3}^{4} y_{12}^{2} y_{20}+\nu^{2} y_{2}^{4} y_{3}^{4} y_{12}^{2} y_{20}+\mu^{\prime} y_{2}^{9} y_{3}^{2} y_{20}^{2} \\
& +\nu^{\prime} y_{2}^{3} y_{3}^{2} y_{12} y_{20}^{2}+y_{12}^{2} y_{20}^{2}+y_{2}^{2} y_{20}^{3} \\
= & 0 .
\end{aligned}
$$

Hence the action of $S q^{4}$ up to the top dimension is zero, hence $w_{4}=0$. Then $w_{5}=w_{6}=w_{7}=0$ as well.

\subsubsection{Potential 4-orientability}

Theorem 2.15. $(\mathbb{O} \otimes \mathbb{H}) \mathbb{P}^{2}$ is 4-orientable if $\beta^{\prime \prime}+\nu^{\prime \prime}=1$.

Proof. $H^{56}\left((\mathbb{O} \otimes \mathbb{H}) \mathbb{P}^{2} ; \mathbb{Z} / 2\right)$ has two generators. Generator $y_{2}^{12} y_{12} y_{20}$ has a unique monomial representing it. The other generator has five representing monomials, one of which reads $y_{2}^{9} y_{3}^{2} y_{12} y_{20}$. Expanding the Cartan formula and substituting in lower squares in Mathematica yields: 


$$
\begin{aligned}
S q^{8}\left(y_{2}^{9} y_{3}^{2} y_{12} y_{20}\right) & =0 \\
S q^{8}\left(y_{2}^{12} y_{12} y_{20}\right) & =\left(1+\beta^{\prime \prime}+\nu^{\prime \prime}\right) y_{2} y_{20}^{3},
\end{aligned}
$$

Hence $S q^{8}$ vanishes on both generators of $H^{56}\left((\mathbb{O} \otimes \mathbb{H}) \mathbb{P}^{2} ; \mathbb{Z} / 2\right)$ precisely if $\beta^{\prime \prime}+\nu^{\prime \prime}=1$, in which case $(\mathbb{O} \otimes \mathbb{H}) \mathbb{P}^{2}$ is 4-orientable.

The coefficient $\beta^{\prime \prime}$ is the prefactor of $y_{2} y_{3}^{2} y_{12}$ in $S q^{8} y_{12}$ and $\nu^{\prime \prime}$ is the prefactor of $y_{2} y_{3}^{2} y_{20}$ in $S q^{8} y_{20}$. 


\section{Part II}

\section{Homotopy type of the space of nested manifolds}




\section{Chapter 3}

\section{Overview of program of studying nested manifolds and cobordisms}

\subsection{Introduction and background to the study of nested manifolds and cobordisms}

\subsubsection{Nested manifolds and cobordisms}

The classical notion of cobordism groups, of which elements are given by manifolds up to cobordism, can be viewed as the connected components, $\pi_{0}$, of a cobordism category. Cobordism categories have as their objects closed manifolds of a certain dimension and morphisms are given by cobordisms between the objects. The higher homotopy groups of cobordism categories extend the notion of cobordism groups, and they were computed in the influential pa- 
3.1 Introduction and background to the study of nested manifolds and cobordisms

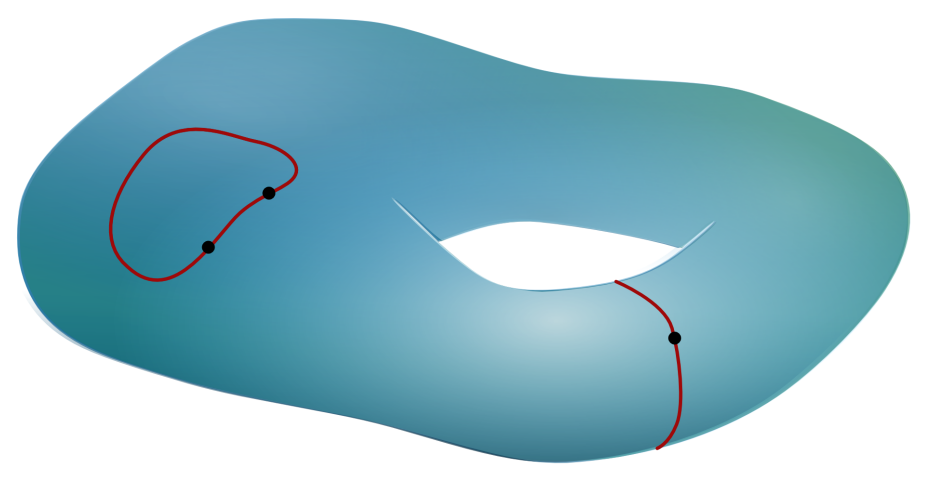

Figure 3.1: An example of a nested manifold $M_{0<1<2}$ : a surface endowed with a 1-dimensional submanifold, which is endowed with a 0-dimensional submanifold.

per [GMTW09] to equal the stable homotopy groups of the Madsen-Tillmann spectrum.

Part II of this thesis concerns the study of spaces of nested manifolds. This forms a foundational part in the study of nested cobordism categories, which are objects that generalise the usual cobordism categories studied in GMTW09. We give the following definition of a nested manifold, illustrated in Figure 3.1:

Definition 3.1. Given a finite sequence of non-negative integers $I=d_{1}<$ $d_{2}<\ldots<d_{m}$, a nested manifold with dimensions $I$ is an ordered tuple

$$
M_{I}=\left(M_{m}, M_{m-1}, \ldots, M_{1}\right),
$$

where $M_{m}$ is a smooth $d_{m}$-dimensional manifold and for $i<m$, we have that $M_{i}$ is a closed subset of $M_{i+1}$ that is diffeomorphic to a smooth manifold of dimension $d_{i}$ without boundary. Note in particular that if the submanifold $M_{i}$ is the empty set, then so are the submanifolds $M_{i^{\prime}<i}$ of lower dimensions.

Similar and related notions are also known by the name of relative manifolds 
3.1 Introduction and background to the study of nested manifolds and cobordisms

(e.g. Yos17]), stratified manifolds (e.g. [Lur09]), manifolds with singularities or simply manifolds with submanifolds (and potentially subsubmanifolds et cetera). Note that we here consider manifolds without boundary, hence a pair of the form $(M, \partial M)$ is not considered to be a nested manifold. However, a manifold with boundary can be doubled along the boundary, yielding a closed manifold with the original boundary as a submanifold.

The central object of study in chapter 4 is a space of nested manifolds inside $\mathbb{R}^{n}$, referred to as $\Psi_{I}\left(\mathbb{R}^{n}\right)$, of which we shall determine the homotopy type. We here give the definition of $\Psi_{I}\left(\mathbb{R}^{n}\right)$ as a set, and it will be endowed with a topology in 4 .

Definition 3.2. Let $I$ be the list $d_{1}<d_{2}<\ldots<d_{m}$, with $d_{1} \geq 0$ and $d_{m}<n$. We define $\Psi_{I}\left(\mathbb{R}^{n}\right)$ to be the set of $m$-tuples of subsets

$$
M_{I}=\left(M_{m}, M_{m-1}, \ldots, M_{1}\right),
$$

of $\mathbb{R}^{n}$, that are nested in the sense that $M_{m} \supset \ldots \supset M_{2} \supset M_{1}$, and for each $i, M_{i}$ is a smooth $d_{i}$-dimensional submanifold of $\mathbb{R}^{n}$ without boundary and closed as a subset of $\mathbb{R}^{n}$.

The set $\Psi_{I}\left(\mathbb{R}^{n}\right)$ equals the set of nested manifolds $M_{I^{\prime}}$ where $I^{\prime}=I<n$ and $M_{m+1}=\mathbb{R}^{n}$.

Studying the space of nested manifolds conjecturally relates to the study of nested cobordism categories in ways that are explained in detail in sections 3.2 and 3.3. The following can be thought of as an intuitive definition of a nested cobordism category, illustrated in Figure 3.2 .

Predefinition 3.3. For non-negative integers $k<d$, a once nested cobordism 
3.1 Introduction and background to the study of nested manifolds and cobordisms

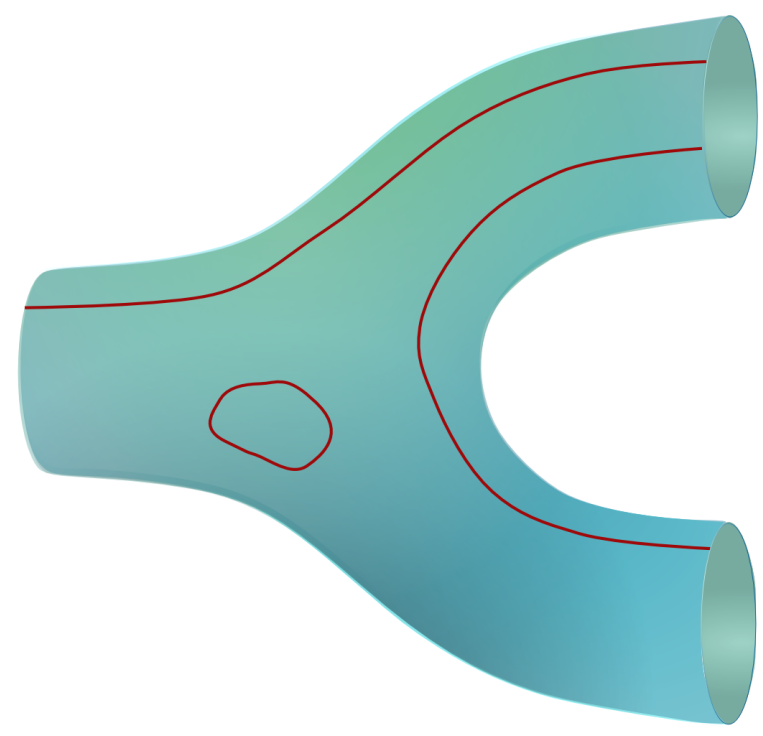

Figure 3.2: Example of a morphism in $\mathcal{C}_{1<2}$.

category $\mathcal{C}_{k<d}$ is a category with:

- An object in $\mathcal{C}_{k<d}$ is given by a $(d-1)$-dimensional manifold $M$ with a $(k-1)$-dimensional submanifold $N$.

- A morphism from $(M, N)$ to $\left(M^{\prime}, N^{\prime}\right)$ is a pair $(W, V)$ where $W$ is a ddimensional cobordism between $M$ and $M^{\prime}, V$ is a $k$-dimensional cobordism between $N$ and $N^{\prime}$, and $V$ is a submanifold of $W$.

To define higher nested cobordism category $\mathcal{C}_{I}$ for a sequence of dimensions $I$ as before, the submanifolds and subcobordisms are again endowed with subsubmanifolds and subsubcobordisms, et cetera.

\subsubsection{Cobordism categories with additional structure}

A number variants of cobordism categories with additional structure have been studied before. In [RW11] an embedded cobordism category $\mathcal{C}_{d}(M)$ is stud- 
3.1 Introduction and background to the study of nested manifolds and cobordisms

ied, with as objects $(d-1)$-dimensional manifolds embedded in some fixed background manifold $M$, and morphisms cobordisms inside $M \times[0,1]$. For every choice of $n$-dimensional manifold $M$, this category forms a subcategory of the nested cobordism category $\mathcal{C}_{d<n}$. Other examples of cobordism categories with additional structure or constraints include the work by Genauer studying cobordisms with specific types of corners (Gen12]), and by Perlmutter considering cobordisms with Baas-Sullivan singularities ([Per14, Per15]). In each of these cases, the homotopy type of these categories is established.

Ayala in his thesis studied a general method using equivariant sheaves to prescribe "geometric structures" on the cobordism category and describe the homotopy type of the resulting cobordism categories with additional structure ([Aya08 $)$. One instance of such an additional structure corresponds to the once nested cobordism category. The project of establishing the homotopy type of nested cobordism categories that part II of this thesis fits into (see section 3.2 will recover Ayala's result with a different proof strategy, based on GRW10]. It will furthermore refine this result by considering a cobordism category internal to $\mathbb{R}^{n}$ rather than $\mathbb{R}^{\infty}$, as well as treating the case of higher nestings.

\subsubsection{Applications of nested cobordism categories}

A main application of nested cobordism categories is the study of defects in topological quantum field theories (TQFTs). TQFTs in physics model systems in which transition amplitudes only depend on topological properties of the system, which can happen in supercooled systems displaying superconductivity. A quantum field theory at higher temperatures will depend on geometry, 
3.1 Introduction and background to the study of nested manifolds and cobordisms

but it is difficult to define a rigorous mathematical framework for such theories. A TQFT with domain a nested cobordism category provides an intermediate point of view by introducing additional content to the system without losing the benefit of the rigorous TQFT description of quantum field theory. On the side of physics this could be interpreted as the introduction of propagating defects in the theory.

TQFTs with singularities or defects represented by embedded subcobordisms are already being studied in mathematical physics, particularly in the case of one-dimensional cobordisms inside two-dimensional ones, i.e. TQFTs with domain $\mathcal{C}_{1<2}$ (see for example [CRS17]). Establising the homotopy type of nested cobordism categories can contribute to the study of invertible TQFTs with defects, in similar way as the homotopy type of the usual cobordism category has been used for the classication of invertible TQFTs by Freed and Hopkins ([FH16]).

The classification of TQFTs of a certain dimension commonly identifies a presentation of a cobordism category and shows that the cutting and gluing of manifolds corresponds to a certain algebraic structure (e.g. [Koc03, SP11, BDSPV15, Juh14]). A similar process is feasible for low-dimensional nested cobordism categories. Indeed it appears that there are interesting algebraic structures that correspond to TQFTs with a nested cobordism category as a domain. For example, one can consider the nested cobordism category $\mathcal{C}_{1<2}$ with one-dimensional cobordisms inside two-dimensional cobordisms as morphisms, and restrict to a subcategory of genus zero morphisms with one incoming boundary circle per connected component. A TQFT defined on this subcategory appears to correspond to a planar algebra as described by Jones 
3.1 Introduction and background to the study of nested manifolds and cobordisms

[Jon99, see also [Yos17].

The nested cobordism category is also of interest for a number of purely topological reasons. Manifolds with specified submanifolds and their cobordisms are studied in low-dimensional topology, for example in knot theory. Indeed, cobordism and concordance of knots form subcategories of the nested cobordism category $\mathcal{C}_{2<4}$. In the nested cobordism category $\mathcal{C}_{0<n}$, cobordisms are endowed with configurations and hence there is a link to configuration spaces. Homological stability of configurations in a fixed manifold has been studied in [CMP15]. Considering $\mathcal{C}_{2<n}$, i.e. choosing our submanifolds to be surfaces, relates the study of nested cobordism categories to the results on homological stability for embeddings of a surface into a fixed manifold modulo diffeomorphisms of the surface by [CMRW17]. Here the stabilisation map is given by increasing the genus of the surface. These results give credibility to the conjecture that homological stability for moduli spaces of embeddings may hold in a more general framework, as discussed in the next section.

Nested cobordism categories also trigger new topological questions. For example, what are the connected components of the nested cobordism categories, nested cobordism groups? A cobordism group $\pi_{0} \mathcal{C}_{k<d}$, with product given by disjoint union on the larger manifold, admits forgetful maps to the cobordism groups $\pi_{0} \mathcal{C}_{d}$ and $\pi_{0} \mathcal{C}_{k}$. While the first map is surjective and split, the second map is less well-behaved, particularly in low codimension. Studying the image of this map may reveal information about embedding dimensions of $k$-dimensional manifolds up to cobordism. 


\subsection{Nested cobordism categories: the overar- ching program}

Part II] of this thesis describes partial results that fit into a larger overarching project studying nested cobordism categories, to be continued in the next few years. The first goal in the project is to establish the infinite loop space modeling the homotopy type of these categories. The homotopy type of the once nested category was also studied by Ayala in his unpublished thesis Aya08. Chapter 4 presents a fundamental step in deriving the homotopy type of the once nested cobordism category with background $\mathbb{R}^{n}$, as well as that of the higher nested versions, using methods different from Aya08, that are instead inspired by the proof for the non-nested case described in [GRW10].

Beyond the goal of deriving the homotopy type of nested cobordism categories, the project aims to establish the stable homology of certain moduli spaces of embeddings of manifolds through application of the study of nested cobordism categories, in analogy to the work in GRW14. In that paper, the calculation of the stable homology for moduli spaces of manifolds under repeated connect sum with $S^{d} \times S^{d}$ is based on the method of parametrised surgery. This entails performing a surgery to a submanifold of $\mathbb{R}^{\infty} \times \mathbb{R}$ at $-\infty$ along the direction of composition of the cobordisms, after dragging the appropriate surgery datum there. This procedure is applied to reduce the cobordism category (with highly connective tangential structure) to a monoid with highly connected morphisms, without changing the homotopy type of the category (i.e. of its classifying space). Application of the group completion theorem to this monoid then yields that the "group completed" limit morphism space has 
the same homology as the entire cobordism category.

In the nested cobordism category, moduli spaces of embeddings rather than moduli spaces of manifolds occur as morphism spaces of the category. Particularly, in the once nested cobordism category, a morphism space between a pair $(M, N)$, representing a manifold $M$ with a submanifold diffeomorphic to $N$, and another pair $\left(M^{\prime}, N^{\prime}\right)$, is given by the space

$$
\begin{aligned}
\bigsqcup_{[W],[V]} \mathcal{M}(V, W) & :=\bigsqcup_{[W],[V]}\left(\operatorname{Emb}^{\partial}\left(W, \mathbb{R}^{\infty}\right) \times \operatorname{Emb}^{\partial}(V, W) / \operatorname{Diff}^{\partial}(V)\right) / \operatorname{Diff}^{\partial}(W) \\
& =\bigsqcup_{[W],[V]} \operatorname{Diff}^{\partial}(V) \backslash \operatorname{Emb}^{\partial}(V, W) / / \operatorname{Diff}^{\partial}(W)
\end{aligned}
$$

Here the disjoint union is taken over diffeomorphism classes $[W]$ of cobordisms between $M$ and $M^{\prime}$ and diffeomorphism classes $[V]$ of cobordisms between $N$ and $N^{\prime}$. A term in the disjoint union corresponds to the space of submanifolds of $\mathbb{R}^{\infty}$ diffeomorphic to $W$, containing a submanifold diffeomorphic to $V$. $\operatorname{Emb}^{\partial}(V, W)$ is the space of embeddings of $V$ into $W$ such that the image of the boundary of $V$ under the embedding corresponds to the submanifolds $N$ in $M$ and $N^{\prime}$ in $M^{\prime}$. This embedding space may be empty, in which case the cartesian product on the first line is empty. The quotient by $\operatorname{Diff}^{\partial}(W)$ identifies $(\phi,[\psi])$ with $\left(\phi \circ f, f^{-1} \circ[\psi]\right)$ for $\phi \in \operatorname{Emb}^{\partial}\left(W, \mathbb{R}^{\infty}\right),[\psi] \in \operatorname{Emb}^{\partial}(V, W) / \operatorname{Diff}^{\partial}(V)$ and $f \in \operatorname{Diff}^{\partial}(W)$. Indeed $(\phi,[\psi])$ and $\left(\phi \circ f, f^{-1} \circ[\psi]\right)$ give rise to the same nested pair of submanifolds of $\mathbb{R}^{\infty}$ for all $f \in \operatorname{Diff}^{\partial}(W)$.

Because moduli spaces of embeddings appear as morphisms spaces in the nested cobordism category, applying a procedure analogous to the one in GRW14 has the potential to relate the homology of these moduli spaces 
to the homology of the nested cobordism category. This may lead to insights about embedding spaces, since the homology of the nested cobordism category is computable.

The stabilization procedure in the classical case corresponds to repeatedly connect summing manifolds a manifold of the form $\left(S^{d} \times S^{d}\right)$. One might therefore conjecture that in the once nested case we can stabilize by connect summing with a nested pair $\left(S^{d} \times S^{d}, S^{k} \times S^{k}\right)$, with a given embedding. In order to apply a similar methodology of reducing the entire nested cobordism category to a submonoid, one should construct a version of parameterised surgery for nested cobordisms. This will be subject to certain dimension restrictions. It will make use of recent generalisations of classical theorems such as the transversality theorem and separation of Morse points to a situation of nested or relative manifolds by Yoshida ([Yos17]).

In [GRW17, GRW] Galatius and Randal-Williams moreover prove a homological stability result for the relevant stabilisation procedure. This tells us that at a given finite homological grading, the homology up to this grading of the cobordism category agrees with the homology of $B \operatorname{Diff}\left(\#^{g} S^{n} \times S^{n}, D^{2 n}\right)$ for some finite $g$. This result can be used to compute characteristic classes of manifold bundles with fibre $\#^{g} S^{n} \times S^{n}$, and a similar result holds for manifold bundles with fibre $W \#^{g} S^{n} \times S^{n}$ for any $2 n$-manifold $W$. This series of papers by Galatius and Randal-Williams has been very influential in the field of algebraic topology of manifolds in recent years.

If the nested cobordism category admits reduction to a suitable submonoid by means of nested parameterised surgery without changing the homotopy type of the category, then this would yield a proof for the following conjecture. Let 
$V_{g}=\#^{g}\left(S^{k} \times S^{k}\right)$ and $W_{g}=\#^{g}\left(S^{d} \times S^{d}\right)$.

Conjecture 3.4. Let $\mathcal{C}_{2 k<2 d}^{\theta}$ be the once nested cobordism category with sufficiently highly connected tangential structure $\theta$. Then for certain $k$ and $d$, there is a homology isomorphism

$$
\underset{g \rightarrow \infty}{\operatorname{hocolim}} \mathcal{M}\left(V_{g} ; W_{g}\right) \longrightarrow \Omega B \mathcal{C}_{2 k<2 n}^{\theta}
$$

As in the case of the normal cobordism category, one then hopes to furthermore prove homological stability. If such a result holds, then the homology of this limit space corresponds to the homology of concrete moduli spaces of embeddings in low degrees. The framework for proving homological stability outlined in [Kra17] can be applied to this situation.

\subsection{How the space of manifolds relates to the cobordism category}

We can think of the morphisms of a cobordism category as manifolds embedded in $\mathbb{R}^{\infty}$ that are "long" in the direction of composition and compact in all other directions. This relates the classifying space of the category, where we forget the directions of morphisms and only consider its composition structure, to the space of all manifolds embedded in $\mathbb{R}^{\infty}$. The space of $d$-dimensional submanifolds of $\mathbb{R}^{n}$ is referred to as $\Psi_{d}\left(\mathbb{R}^{n}\right)$ (see Definition 4.4 for a precise definition as a set and Definition 4.6 for a description of an appropriate topology). The relationship between $\Psi_{d}\left(\mathbb{R}^{n}\right)$ and the cobordism category with $d$-dimensional morphisms was made precise in [GMTW09] and later in [GRW10]. We de- 
fine $\psi_{d}(n, k) \subset \Psi_{d}\left(\mathbb{R}^{n}\right)$ as the subset of those manifolds that are embedded in $(-1,1)^{n-k} \times \mathbb{R}^{k}$

Theorem 3.5 ([GRW10]). We have the following homotopy equivalences:
(A) $B \mathcal{C}_{d}\left(\mathbb{R}^{n}\right) \simeq \psi_{d}(n, 1)$
(B) $\psi_{d}(n, 1) \simeq \Omega^{n-1} \psi_{d}(n, n)=\Omega^{n-1} \Psi_{d}\left(\mathbb{R}^{n}\right)$
(C) $\Psi_{d}\left(\mathbb{R}^{n}\right) \simeq T h\left(\gamma_{d, n}^{\perp}\right)$

Which together amount to:

$$
B \mathcal{C}_{d}\left(\mathbb{R}^{n}\right) \simeq \Omega^{n-1} T h\left(\gamma_{d, n}^{\perp}\right)
$$

A similar setup can be applied to determine the homotopy type of the realisations of nested cobordism categories $B \mathcal{C}_{k<d}$ or $B \mathcal{C}_{I}$, which are not defined in detail in this thesis. In this thesis the equivalent statement of Theorem 3.5 (C) is proved for the case of nested manifolds. This is presumably the step where the proof of a theorem analogous to Theorem 3.5 for nested cobordism categories will differ most fundamentally from the original proof. 


\section{Chapter 4}

\section{Homotopy type of the space of nested manifolds}

\subsection{Introduction}

In GMTW09, Galatius, Madsen, Tillmann and Weiss identified the homotopy type of the classifying space of the cobordism category with objects $d$-1-dimensional manifolds embedded in $\mathbb{R}^{\infty}$. This result was refined by Galatius and Randal-Williams in GRW10 to the case of a cobordism category subordinate to $\mathbb{R}^{n}$, where the objects are $d$-1-manifolds embedded in $\mathbb{R}^{n-1}$ and the morphisms are $d$-dimensional cobordisms inside $\mathbb{R}^{n}$ for some finite $n$. One key step in the proof of the latter is to show that the space $\Psi\left(\mathbb{R}^{n}\right)$ of $d$-dimensional manifolds in $\mathbb{R}^{n}$ is homotopy equivalent to the Thom space of the bundle $\gamma_{d, n}^{\perp} \rightarrow G r_{d}\left(\mathbb{R}^{n}\right)$, the canonical perpendicular bundle over the Grassmannian of $d$-planes in $\mathbb{R}^{n}$.

Theorem 4.1 (GRW10] Theorem 3.22, reproved here as Theorem 4.12). 
There is a homotopy equivalence

$$
\Psi_{d}\left(\mathbb{R}^{n}\right) \simeq T h\left(\gamma_{d, n}^{\perp}\right)
$$

This chapter discusses the proof of this theorem and proves an analogous theorem in the situation of spaces of nested manifolds inside $\mathbb{R}^{n}$.

Section 4.2 gives a recapitulation of the proof of Theorem 4.12. It focuses in particular on a technical lemma regarding the injectivity radius of the normal exponential map (Lemma 4.10 that can be used to resolve in more detail a step in the proof concerning the set of manifolds with a unique closest point to the origin. A proof of the lemma is given as well as how it fits into the proof of Theorem 4.12

Section 4.3 discusses the homotopy type of the space of once nested manifolds, i.e. the space $\Psi_{k<d}\left(\mathbb{R}^{n}\right)$ of $d$-dimensional manifolds with $k$-dimensional submanifolds inside $\mathbb{R}^{n}$, defined in detail in Definition 4.14. We first discuss a further technical lemma (Lemma 4.13), which is similar in nature to Lemma 4.10, although not entirely analogous. In Definition 4.16 we define a suitable linearised model $T_{k<d}\left(\mathbb{R}^{n}\right)$ for the homotopy type of the space of nested manifolds and prove the statement:

Theorem 4.2 (Theorem 4.17). There is a weak homotopy equivalence

$$
\Psi_{k<d}\left(\mathbb{R}^{n}\right) \simeq T_{k<d}\left(\mathbb{R}^{n}\right)
$$

Section 4.4 generalises to the situation of a space $\Psi_{I}\left(\mathbb{R}^{n}\right)$ of arbitrarily nested manifolds of dimensions $I$ inside $\mathbb{R}^{n}$, where $I$ is a finite list of the form $k_{1}<$ $k_{2}<\ldots<k_{m}$ (Definition 4.19). With an induction on the previous proof we 
show that

Theorem 4.3 (Theorem 4.21). There is a weak homotopy equivalence

$$
\Psi_{I}\left(\mathbb{R}^{n}\right) \simeq T_{I}\left(\mathbb{R}^{n}\right)
$$

Here $T_{I}\left(\mathbb{R}^{n}\right)$ is a linearised model defined in Definition 4.20 .

\subsection{Homotopy type of the space of embedded manifolds}

\subsubsection{Definitions}

Definition 4.4. For $d<n$ non-negative integers, let $\Psi_{d}\left(\mathbb{R}^{n}\right)$ be the set of subsets $M$ of $\mathbb{R}^{n}$, where $M$ is a smooth $d$-dimensional submanifold of $\mathbb{R}^{n}$ without boundary and closed as a subset of $\mathbb{R}^{n}$.

We endow this space with the plot topology $\tau_{S P}$ defined by Schommer-Pries in [SP17], which was shown to be equivalent to the original topology $\tau_{G R W}$ defined in GRW10]. The plot topology is defined using the method of plots: we define a topology on a set $Y$ as the finest topology such that a collection of maps into $Y$, referred to as plots, are continuous.

Consider a space $X$ and a map of sets $f: X \rightarrow \Psi_{d}\left(\mathbb{R}^{n}\right)$. We define the graph of $f$ not in the usual manner but as the following set:

$$
\Gamma(f)=\left\{(x, w) \in X \times \mathbb{R}^{n} \mid w \in f(x) \subset \mathbb{R}^{n}\right\} .
$$

Definition 4.5 ([SP17]). For $N$ a smooth manifold, we define a map $p$ : 
$N \rightarrow \Psi_{d}\left(\mathbb{R}^{n}\right)$ to be a plot if $\Gamma(p)$ is a smooth submanifold of $N \times \mathbb{R}^{n}$ and the projection of $\Gamma(p)$ onto $N$ is a submersion.

Definition 4.6 ([SP17]). Let $\tau_{S P}$ be the finest topology on $\Psi_{d}\left(\mathbb{R}^{n}\right)$ such that for all smooth manifolds $N$, plots $p: N \rightarrow \Psi\left(\mathbb{R}^{n}\right)$ are continuous.

This topology has the property that a compact manifold can be pushed off to infinity in a continuous manner. In other words, if $M \in \Psi_{d}\left(\mathbb{R}^{n}\right)$ is a compact manifold, then there exists a continuous path $\gamma:[0,1] \rightarrow \Psi_{d}\left(\mathbb{R}^{n}\right)$ such that $\gamma(0)=M$ and $\gamma(1)=\varnothing$. Another useful property of this topology on the space of manifolds is that it acts as a sheaf of topological spaces over $\mathbb{R}^{n}$. For $U$ and open subset of $\mathbb{R}^{n}, \Psi_{d}(U)$ is the space of smooth $d$-dimensional submanifold of $\mathbb{R}^{n}$ without boundary and closed as a subset of $U$, topologized analogously to $\Psi_{d}\left(\mathbb{R}^{n}\right)$. We have the following theorem:

Theorem 4.7 (GRW10] Theorem 2.11). For $U, V$ open subsets of $\mathbb{R}^{n}$, the map

$$
\operatorname{Emb}(U, V) \times \Psi_{d}(U) \rightarrow \Psi_{d}(V)
$$

given by $(j, M) \mapsto j^{-1}(M)$, is continuous.

This implies in particular that any self-diffeomorphism of $\mathbb{R}^{n}$, or embedding of $\mathbb{R}^{n}$ into itself, induces a continous map on $\Psi_{d}\left(\mathbb{R}^{n}\right)$.

\subsubsection{Technical lemma concerning the size of tubular neighbourhoods}

Consider a manifold $M \in \Psi_{d}\left(\mathbb{R}^{n}\right)$. The normal bundle $\nu_{M}$ is an $n-d$ dimensional vector bundle over $M$ that is given fiberwise by the complement 
of the tangent space $T_{x} M$ as a subspace of $T_{x} \mathbb{R}^{n}$, which inherits a natural metric from $\mathbb{R}^{n}$. The isometric map $\nu_{M} \rightarrow \mathbb{R}^{n}$ that takes vectors in the normal bundle at $x \in M$ to actual affine vectors in $\mathbb{R}^{n}$ normal to $T_{x} M$ is called the (normal) exponential map. It is the restriction of the exponential map of $\mathbb{R}^{n}$ to $\nu_{M} \subset T \mathbb{R}^{n}$. The exponential map identifies the zero section of $\nu_{M}$ with $M \subset \mathbb{R}^{n}$.

Every manifold $M \in \Psi_{d}\left(\mathbb{R}^{n}\right)$ admits a tubular neighbourhood $N(M)$ (for reference see for example [Lee03], chapter 10). This is an open neighbourhood of the zero section of the normal bundle on which the exponential map restricts to an embedding, with image $\tilde{N}(M) \subset \mathbb{R}^{n}$ an open neighbourhood of $M$ in $\mathbb{R}^{n}$.

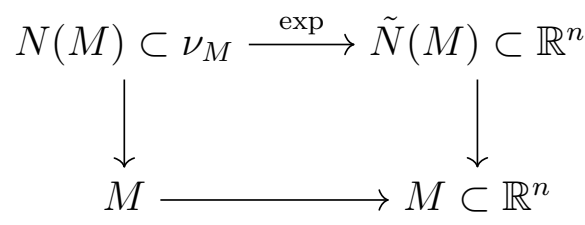

For a compact manifold, this tubular neighbourhood can be chosen to have uniform width, i.e. $N(M)$ can be chosen to be a disc bundle with fiber a ball of radius $\varepsilon$ for some small enough $\varepsilon>0$. More generally, we can choose $N(M)$ to be a disc bundle with varying radius $\varepsilon(x)>0$.

Consider $\Gamma_{c}(N(M))$ the space of compactly supported sections of the normal bundle $\nu_{M}$ taking values in $N(M) \subset \nu_{M}$, endowed with the strong Whitney $C^{\infty}$ topology (see for example [Mic80]). The normal exponential map now extends to a map $\Gamma_{c}(N(M)) \rightarrow \Psi_{d}\left(\mathbb{R}^{n}\right)$ realising a section of the normal bundle as an embedded manifold inside $\tilde{N}(M) \subset \mathbb{R}^{n}$. We will use the following result relating open sets in $\Psi_{d}\left(\mathbb{R}^{n}\right)$ with open sets in $\Gamma_{c}(N(M))$. 
Lemma 4.8 (GRW10, see also [CMRW17, SP17]). Let $M \in \Psi_{d}\left(\mathbb{R}^{n}\right)$, and choose a tubular neighbourhood $N(M) \subset \nu_{M}$. Given a compact subset $K \subset \mathbb{R}^{n}$ and an open set $U \subset \Gamma_{c}(N(M))$, the set $M_{K, U}$ of manifolds $M^{\prime}$ such that $M^{\prime} \cap K=\exp _{M}(f(M)) \cap K$ for a section $f \in U$, is open in $\Psi_{d}\left(\mathbb{R}^{n}\right)$.

Let $\Psi_{d}^{0} \subset \Psi_{d}\left(\mathbb{R}^{n}\right)$ be the subspace of manifolds containing 0 . We will use the choice of a small enough neighbourhood $U \subset \Gamma_{c}(N(M))$ to argue that we can make the choice of radius for the tubular neighbourhood locally constant inside $\Psi_{d}^{0}$

Lemma 4.9. For every $M \in \Psi_{d}^{0}$ there exists an open neighbourhood $V \subset \Psi_{d}^{0}$ of $M$ and an $\varepsilon>0$ such that for every $M^{\prime} \in V, M^{\prime} \cap \bar{B}_{1}(0)$ admits an $\varepsilon$-tubular neighbourhood.

Proof. Note first that for any $r>0, M \cap \bar{B}_{r}(0)$ admits tubular neighbourhood of constant width. Choose $r=2$ and call the width of the tubular neighbourhood $\varepsilon^{\prime}$.

We will construct the neighbourhood $V$ of $M$ by choosing $U \subset \Gamma_{c}(N(M))$ in Lemma 4.8 to be a small open $\delta$-ball in the Whitney $C^{2}$ topology for some sufficiently small $1>\delta>0$. This implies in particular that we restrict the absolute values of the section and its first and second order partial derivatives to be smaller than $\delta$. As $\delta<1=r-1$, exponentiating the sections does not move points of $M$ outside the $\bar{B}_{r}(0)$ ball into the $\bar{B}_{1}(0)$ ball.

The maximal width of a tubular neighbourhood of $M^{\prime}=\exp _{M}(f(M))$ is determined by the distance to its medial axis or cut locus in $\mathbb{R}^{n}$, defined as the collection of points that are equidistant to two or more points of $M^{\prime}$ (for example see [MM02]). In other words, the medial axis is given by points where 
the normal exponential map restricted to vectors of length $l$ is not injective, for a given $l$. See figure 4.1 for illustration.

A point may belong to the medial axis of $M^{\prime}$ either for local or for global reasons (or both). A point $p \in \mathbb{R}^{n}$ may locally be on the medial axis because it is a focal point of a point $m$ on the manifold. This means that there is a circle in a normal plane at $m$ centered at $p$ that is tangent to the manifold at $m$ up to second order, hence locally there are normal rays originating from the manifold in a neighbourhood of $m$ that focus at $p$. Alternatively, the manifold may on a global scale curve back on itself such that two points on the manifold that are distant along the manifold are close to each other within the encompassing $\mathbb{R}^{n}$.

Given that $M$ admits an $\varepsilon^{\prime}$-tubular neighbourhood, by restricting both the value and the first derivatives of a section $f$, we can make sure that no new global points in the medial axis appear at a distance less than $\varepsilon^{\prime} / 2$ away from $M^{\prime} \cap \bar{B}_{1}(0)=\exp _{M}(f(M)) \cap \bar{B}_{1}(0)$.

The appearance of focal points is determined by the second fundamental form $\alpha(x, y)$. In particular, for $v \in T_{m} M$, a geodesic through $m$ in the direction of $v$ can locally be written as ([MMASC14])

$$
\gamma_{v}(t)=m+v t+\alpha_{M}(v, v) t^{2}+O\left(t^{3}\right)
$$

Thus, a geodesic on $M$ infinitesimally within $\mathbb{R}^{n}$ looks like a parabola with a focal point determined by $\alpha_{M}$. The second fundamental form of the exponentiated section $M^{\prime}, \alpha_{M^{\prime}}$, is determined by $\alpha_{M}$ and in a continuous way by the section and its first and second partial derivatives. Hence, by restricting the values of the second order derivatives we can accomplish that no new focal 


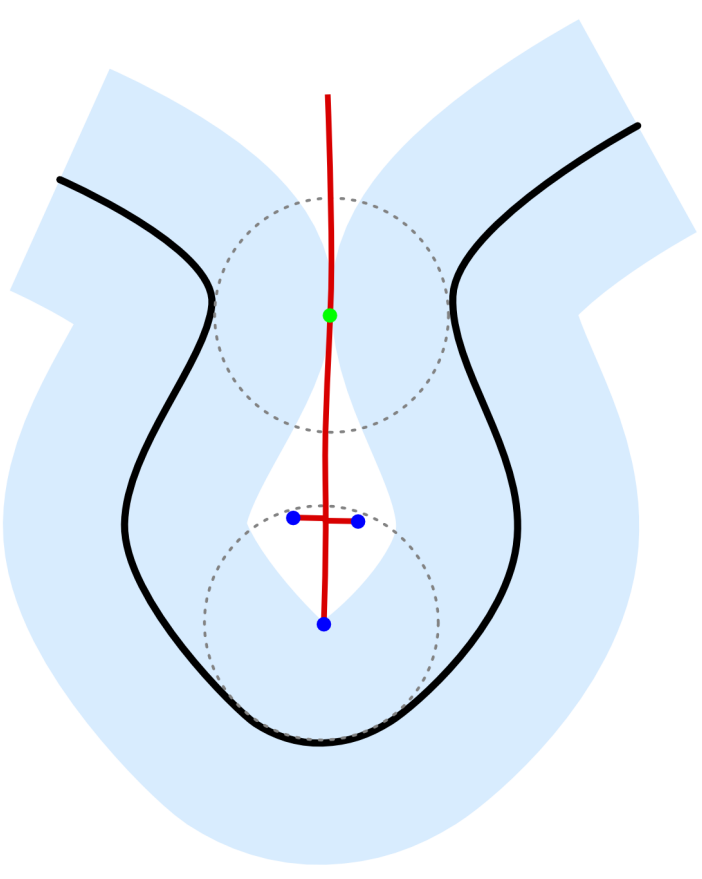

Figure 4.1: Example of the medial axis (red) to a manifold (black), together with a maximal tubular neighbourhood (light blue) with radius determined by the minimal distance between the manifold and its medial axis. All points of the medial axis can be thought of as lying at the centre of a circle that is tangent to the manifold at two or more points. The dark blue points belongs to the medial axis for local reasons because these are focal points of the manifold: centres of circles that are tangent to the manifold to second order. The green point, as well as other points in this example, belong to the medial axis for global reasons, lying at the centre of a circle that touches the manifold at distinct points. 
points appear within a distance $\varepsilon^{\prime} / 2$ of $M^{\prime}$.

Hence, by choosing $\delta$ small enough, we obtain that $M^{\prime} \cap \bar{B}_{1}(0)$ admits an $\varepsilon:=\varepsilon^{\prime} / 2$-tubular neighbourhood.

Lemma 4.10. There exists a continuous map $\varepsilon: \Psi_{d}^{0} \rightarrow(0,1)$ such that for all $M \in \Psi_{d}^{0}\left(\mathbb{R}^{n}\right), M \cap \bar{B}_{1}(0)$ admits an $\varepsilon(M)$-tubular neighbourhood.

Proof. Lemma 4.9 yields a cover of $\Psi_{d}^{0}$ of open neighbourhoods $V_{M}$, for $M \in$ $\Psi_{d}^{0}$, together with constant functions $V_{M} \rightarrow \varepsilon_{M}$ with property that $M^{\prime} \cap \bar{B}_{1}(0)$ admits an $\varepsilon(M)$-tubular neighbourhood for all $M^{\prime} \in V_{M}$. As $\Psi_{d}^{0} \subset \Psi_{d}\left(\mathbb{R}^{n}\right)$ is metrizable ([BM14, CMP15]), hence paracompact, we are allowed to take a locally finite refinement of this cover (see [Mun00], §41). Using a partition of unity subordinate to the new cover, we glue the constant functions $V_{M} \rightarrow \varepsilon_{M}$, thus constructing a continuous function $\varepsilon: \Psi_{d}^{0} \rightarrow(0,1)$, with the property that $M \cap \bar{B}_{1}(0)$ admits an $\varepsilon(M)$-tubular neighbourhood.

Let $\nu_{0}$ be the vector bundle over $\Psi_{d}^{0}$ with fiber over $M$ given by the normal bundle to $M$ at 0 . Lemma 4.10 implies that for $v \in \nu_{0, M}$ with $\|v\| \leq \varepsilon(M)$, $v$ has a unique point in $M$ closest to it, and this point is 0 . Equivalently, the shifted manifold $M+v$ has the property that $v$ (now lying on $M$ ) is the unique closest point of $M$ to 0 . We define $e: \nu_{0} \rightarrow \Psi_{d}\left(\mathbb{R}^{n}\right)$ to be the map that takes $(M, v)$ to $M+v$. Let $D_{\varepsilon}\left(\nu_{0}\right)$ be the disc bundle inside $\nu_{0}$ with fiber over $M$ the disc of radius $\varepsilon(M)$ normal to $M$ at 0 . Then Lemma 4.10 implies that the map $e$ is injective on $D_{\varepsilon}\left(\nu_{0}\right)$.

In the rest of this chapter we will assume that the tubular neighbourhood given by $\varepsilon$ is not the maximal, in the sense that the map $e$ is still injective on the sphere bundle $S_{\varepsilon}\left(\nu_{0}\right)$. If this were not the case then we could replace $\varepsilon$ with a map $\tilde{\varepsilon}: \Psi_{d}^{0} \rightarrow(0,1)$ that is strictly dominated by $\varepsilon$. 
Lemma 4.11. The map e $: \bar{D}_{\varepsilon}\left(\nu_{0}\right) \rightarrow \Psi_{d}\left(\mathbb{R}^{n}\right)$ is an open map.

Proof. Take $(M, v) \in \stackrel{\circ}{D}_{\varepsilon^{\prime}}\left(\nu_{0}\right)$, where $\varepsilon^{\prime}$ is a continuous function satisfying the condition in Lemma 4.10 and dominating $\varepsilon$. For $U \subset \stackrel{\circ}{D_{\varepsilon}^{\prime}}\left(\nu_{0}\right)$ an open neighbourhood of $(M, v)$, we will construct a neighbourhood $V$ of $e(M, v)=$ $M+v$ such that $V \subset e(U)$. We first make a choice of tubular neighbourhood $N(M)$ with radius less than $\varepsilon(M)$ around $M$ and then choose to consider $N(M)+v$ as a tubular neighbourhood of $M+v$. The bundle $\bar{D}_{\varepsilon}\left(\nu_{0}\right)$ is locally trivialisable, hence there exists a $0<\delta<\varepsilon^{\prime}(M)$ such that

$$
(M, v) \in U_{\delta}^{\Psi_{d}^{0}} \times \stackrel{\circ}{B}_{\delta}(v) \subset U
$$

where $U_{\delta}^{\Psi_{d}^{0}}$ is an open neighbourhood of $M$ in $\Psi_{d}\left(\mathbb{R}^{n}\right)$ as discussed in Lemma 4.8 intersected with $\Psi_{d}^{0}$, given by exponentiating sections in the tubular neighbourhood of $M$ that are $\delta$-small in the $C^{\infty}$ topology (the value and all derivatives are bound by $\delta$ ) and have value 0 at 0 , and

$$
\stackrel{\circ}{B}_{\delta}(v):=\left\{w \in \mathbb{R}^{n-d}|| w-v \mid<\delta\right\} .
$$

The image of $U_{\delta}^{\Psi_{d}^{0}} \times \stackrel{\circ}{B}_{\delta}(v)$ under $e$ contains the open neighbourhood $V_{d}^{\Psi_{d}}$ given by by exponentiating sections in the tubular neighbourhood of $M+v$ that are $\delta$-small in the $C^{\infty}$ topology. Hence we have constructed an open set $V:=V_{d}^{\Psi_{d}}$ such that $M+v \in V \subset e(U)$. The map $e$ is therefore open on $\stackrel{\circ}{D}_{\varepsilon^{\prime}}\left(\nu_{0}\right)$ hence in particular on $\bar{D}_{\varepsilon}\left(\nu_{0}\right)$.

\subsubsection{Homotopy type of the space of manifolds}

We will now apply Lemma 4.10 in the proof of Theorem 4.12 below. 
Theorem 4.12 (GRW10]). There is a homotopy equivalence

$$
\Psi_{d}\left(\mathbb{R}^{n}\right) \simeq T h\left(\gamma_{d, n}^{\perp}\right)
$$

Reformulated proof. There is an injective map $q: T h\left(\gamma_{d, n}^{\perp}\right) \hookrightarrow \Psi_{d}\left(\mathbb{R}^{n}\right)$ defined by $q(\infty)=\varnothing ; q(P, v)=P+v$, by which we mean $P \in G r_{d}\left(\mathbb{R}^{n}\right)$ realised as a plane in $\mathbb{R}^{n}$ shifted by the normal vector $v$ away from the origin. Restricted to the zero-section $G r_{d}\left(\mathbb{R}^{n}\right) \subset T h\left(\gamma_{d, n}^{\perp}\right), q$ lands in $\Psi_{d}^{0}$. Call its image $L_{0}=q\left(G r_{d}\left(\mathbb{R}^{n}\right)\right)$, the space of linear manifolds. First we show that $L_{0}$ is a deformation retract of $\Psi_{d}^{0}$. Let $T_{t}: \mathbb{R}^{n} \rightarrow \mathbb{R}^{n}$ be multiplication by $\frac{1}{1-t}$, which is a diffeomorphism of $\mathbb{R}^{n}$ for $t \neq 1$. Define the homotopy $F: \Psi_{d}^{0} \times[0,1] \rightarrow \Psi_{d}^{0}$ by

$$
F(M, t)= \begin{cases}T_{t}(M) & 0 \leq t<1 \\ T_{0} M & t=1\end{cases}
$$

This is a continuous map and it restricts to the identity on $L_{0}$, hence $\Psi^{0} \simeq$ $L_{0} \cong G r_{d}\left(\mathbb{R}^{n}\right)$.

Let $\nu_{0}$ be the $(n-d)$-dimensional vector bundle over $\Psi_{d}^{0}$ given over $M$ by the normal bundle to $M$ at 0 , i.e. $\nu_{0, M}=\nu_{M, 0}$. We can define a continuous map $e: \nu_{0} \rightarrow \Psi_{d}\left(\mathbb{R}^{n}\right)$ given by $(M, v) \mapsto M+v$, the translate of $M$ by $v$. Over the deformation retract $L_{0} \subset \Psi_{d}^{0}, \nu_{0}$ restricts to $q_{*}\left(\gamma_{d, n}^{\perp}\right)$. Restricted to $\|v\| \leq \varepsilon(M)$ as defined in Lemma 4.10, the map $e$ is injective. Hence the disc bundle $D_{\varepsilon(M)} \nu_{0}$ embeds into $\Psi_{d}\left(\mathbb{R}^{n}\right)$ as a neighbourhood of $\Psi_{d}^{0}$.

Let $C$ be the complement in $\Psi_{d}\left(\mathbb{R}^{n}\right)$ of $e\left(\bar{D}_{\varepsilon(M)} \nu_{0}\right)$. By applying the homotopy equivalence between $S^{n-1}$ and $D^{n} \backslash\{0\}$ fiberwise, we have that $\partial e\left(\bar{D}_{\varepsilon} \nu_{0}\right) \simeq$ 
$e\left(\bar{D}_{\varepsilon} \nu_{0}\right) \backslash \Psi_{d}^{0}$, hence $C \simeq C^{\prime}=\Psi_{d}\left(\mathbb{R}^{n}\right) \backslash \Psi_{d}^{0}$. $C^{\prime}$ is the set of manifolds that do not contain the origin. Hence $C^{\prime}$ can be contracted to $\varnothing$ by the homotopy $F^{\prime}: C^{\prime} \times[0,1] \rightarrow C^{\prime}$ given by:

$$
F^{\prime}(M, t)= \begin{cases}T_{t}(M) & 0 \leq t<1 \\ \varnothing & t=1 .\end{cases}
$$

We have that $C \subset V$ where $V$ is the complement of $e\left(\bar{D}_{\varepsilon(M) / 2} \nu_{0}\right)$, which is open by Lemma4.11. Applying the homotopy equivalence between the annulus and the circle fiberwise, we see that $C$ is a neighbourhood deformation retract of $V$. Hence collapsing $C$ in $\Psi_{d}\left(\mathbb{R}^{n}\right)$ yields a homotopy equivalent space, which is homeomorphic to the space obtained by collapsing the sphere bundle $S_{\varepsilon(M)} \nu_{0}$, i.e. the Thom space of $\nu_{0} \rightarrow \Psi_{d}^{0}$. This Thom space is homotopy equivalent to $\operatorname{Th}\left(\gamma_{d, n}^{\perp}\right)$.

\subsection{Homotopy type of spaces of once nested manifolds}

\subsubsection{Technical lemma concerning the injectivity radius of the tangential exponential map}

The embedding of a manifold $M$ in $\mathbb{R}^{n}$ naturally endows $M$ with a Riemannian metric. This implies that at every point $x \in M$, we have a tangential exponential map $T_{x} M \rightarrow M$, which is length-preserving on vectors radiating from $0 \in T_{x} M$, which are mapped onto geodesic rays in $M$ radiating from $x$. 
This tangential exponential map is a diffeomorphism in a small neighbourhood of $x$. Explicitly, every point $x$ of a Riemannian manifold admits a radius $r$ such that $\exp : B_{r}(0) \subset T_{x} M \rightarrow M$ is injective.

For the situation of nested manifolds, we will require not only a continuous tubular neighbourhood as prescribed by the function $\varepsilon$ in the previous section, but also a continuously varying tangential neighbourhood.

In order to establish the homotopy type of the space of nested manifolds, we will need to establish that the we can define a radius within which the exponential map at 0 is injective in a continuous way with respect to varying the manifold $M \in \Psi_{d}^{0}$ within the topology on the space of embedded manifolds.

Lemma 4.13. We can define a continuous function $\eta: \Psi_{d}^{0} \rightarrow(0, \infty]$ such that the following conditions are met:

(C1) $\eta$ is smaller than the injectivity radius of the tangential exponential map at 0, i.e. for $T_{0} M^{\eta}=T_{0} M \cap B_{\eta}(0), \exp _{M}: T_{0} M^{\eta} \rightarrow M$ is injective;

(C2) For every $x \in T_{0} M^{\eta}$, the ray $s x+(1-s) \exp (x)$, for $x \in T_{0} M^{\eta}$ and $s \in[0,1]$ intersects $M$ only in $\exp (x)$, where we have identified $x$ with a point in $\mathbb{R}^{n}$ using the inclusion $T_{0} M \subset T_{0} \mathbb{R}^{n}$ and the fact that the exponential map $\exp _{M}: T_{0} \mathbb{R}^{n} \rightarrow \mathbb{R}^{n}$ is a global diffeomorphism;

(C3) All points of $M$ inside the ball of radius $\eta / 2$ in $\mathbb{R}^{n}$ are image points of the exponential map on $T_{0} M^{\eta}$. i.e. $B_{\eta / 2}^{\mathbb{R}^{n}}(0) \cap M \subset \exp _{M}\left(T_{0} M^{\eta}\right)$.

(C4) If $M$ is a plane, i.e. $M \in G r_{d}\left(\mathbb{R}^{n}\right)$, then $\eta=\infty$.

Proof. For any smooth manifold $M$ embedded in $\mathbb{R}^{n}$ it is possible to find a radius $\eta_{M}$ such that conditions $\mathrm{C} 1-\mathrm{C} 3$ are met. If $M$ is a plane then this 
radius can be chosen to equal $\infty$. If $M$ is perturbed with a smooth compactly supported section, then the radius of injectivity of the tangential exponential map at $0(\mathrm{C} 1)$; the radii within which the rays above do not intersect the manifold elsewhere $(\mathrm{C} 2)$; and the radii of a balls in $\mathbb{R}^{n}$ which only contain image points of the exponential map (C3), all vary continuously with respect to the derivatives of the section.

Hence, with a similar argument as provided in Lemma 4.9, we can find an $\eta<$ $\eta_{M}$ and a small enough neighbourhood $V_{M}$ of $M$ consisting of exponentiated sections as in Lemma 4.8, such for each $M^{\prime} \in V_{M}, \eta$ satisfies conditions C1-C3. If $M=P$ a plane, then by restricting derivatives we can find a neighbourhood of compactly supported sections of the normal bundle of $M$ such that exponentiating any of these sections does not violate conditions C1-C3 for $\eta=\infty$. This gives a series of neighbourhoods $V_{P}$ for $P \in G r_{d}\left(\mathbb{R}^{n}\right)$.

We have thus constructed a cover of $\Psi_{d}^{0}$ of open neighbourhoods $V_{M}$, for $M \in$ $\Psi_{d}^{0}$, together with constant functions $V_{M} \rightarrow \eta_{M}$ with the desired property. We take a locally finite refinement of this cover, making sure to include all planes in a neighbourhood of the form $V_{P}$.

We can take a local refinement as $\Psi_{d}^{0} \subset \Psi_{d}\left(\mathbb{R}^{n}\right)$ is metrizable ([BM14, CMP15]), hence paracompact. Using a partition of unity subordinate to the new cover, we glue the constant functions $V_{M} \rightarrow \eta_{M}$, thus constructing a continuous function $\eta: \Psi_{d}^{0}\left(\mathbb{R}^{n}\right) \rightarrow(0,1)$, with the property that $\eta(M)$ satisfies conditions C1-C3, and that takes the value $\infty$ on $P \in G r_{d}\left(\mathbb{R}^{n}\right)$. 


\subsubsection{Definitions}

Definition 4.14. For $k<d<n$ non-negative integers, let $\Psi_{k<d}\left(\mathbb{R}^{n}\right)$ be the set of pairs of subsets $(M, N)$ of $\mathbb{R}^{n}$, where $M$ is a smooth $d$-dimensional submanifold of $\mathbb{R}^{n}$ without boundary and closed as a subset of $\mathbb{R}^{n}$ and $N$ is a smooth $k$-dimensional submanifold of $M$, and thus in particular of $\mathbb{R}^{n}$, without boundary and closed as a subset of $M$. This set pointwise includes into the set $\Psi_{d}\left(\mathbb{R}^{n}\right) \times \Psi_{k}\left(\mathbb{R}^{n}\right)$, the product of the sets of manifolds insider $\mathbb{R}^{n}$ of dimension $d$ and $k$ respectively. These sets were endowed with a topology in GRW10. In [SP17] a topology was defined on these spaces using the method of plots, see also Definition 4.6, which was then shown to be equivalent to the original topology. We endow $\Psi_{k<d}\left(\mathbb{R}^{n}\right)$ with the subspace topology from its inclusion in this product, so that a sequence of manifolds $\left(M_{n}, N_{n}\right)$ converges to $(M, N)$ precisely if $M_{n}$ converges to $M$ and $N_{n}$ converges to $N$ in $\Psi_{d}\left(\mathbb{R}^{n}\right)$ and $\Psi_{k}\left(\mathbb{R}^{n}\right)$ respectively.

Since $\Psi_{k<d}\left(\mathbb{R}^{n}\right)$ has the subspace topology, the inclusion $i: \Psi_{k<d}\left(\mathbb{R}^{n}\right) \rightarrow$ $\Psi_{d}\left(\mathbb{R}^{n}\right) \times \Psi_{k}\left(\mathbb{R}^{n}\right)$ is continuous. Hence, by composing this map with the respective projection maps onto the two components, we obtain that the forgetful $\operatorname{maps} F_{d}: \Psi_{k<d}\left(\mathbb{R}^{n}\right) \rightarrow \Psi_{d}\left(\mathbb{R}^{n}\right)$ and $F_{k}: \Psi_{k<d}\left(\mathbb{R}^{n}\right) \rightarrow \Psi_{k}\left(\mathbb{R}^{n}\right)$ are continuous. The first forgetful map is clearly surjective and has a canonical section, given by sending $M$ to $(M, \varnothing)$. The second forgetful map is less well-behaved.

Lemma 4.15. The forgetful map $F_{d}: \Psi_{k<d}\left(\mathbb{R}^{n}\right) \rightarrow \Psi_{d}\left(\mathbb{R}^{n}\right)$ is open.

Proof. Let $V \subset \Psi_{k<d}\left(\mathbb{R}^{n}\right)$ be open. Take $(M, N) \in V$. We will construct an open set $U \subset \Psi_{d}\left(\mathbb{R}^{n}\right)$ such that $M \in U \subset F_{d}(V)$. By the definition of the 
subspace and product topologies, we have that

$$
(M, N) \in V_{d} \times V_{k} \cap \Psi_{k<d}\left(\mathbb{R}^{n}\right) \subset V
$$

for some open sets $V_{d}$ and $V_{k}$ in $\Psi_{d}\left(\mathbb{R}^{n}\right)$ and $\Psi_{k}\left(\mathbb{R}^{n}\right)$ respectively.

By Lemma 4.8, $M$ has a neighbourhood $\tilde{U}_{d}$ that is given by exponentiating compactly supported sections in the tubular neighbourhood $N(M)$. Similarly, $N$ has a neighbourhood $\tilde{U}_{k}$ that is given by exponentiating compactly supported sections in the tubular neighbourhood $N(N)$. Such open sets form a neighbourhood basis (see [CM16]) for the topologies on $\Psi_{d}\left(\mathbb{R}^{n}\right)$ and $\Psi_{k}\left(\mathbb{R}^{n}\right)$, hence I can choose these open sets small enough such that $\tilde{U}_{d} \subset V_{d}$ and $\tilde{U}_{k} \subset V_{k}$. Furthermore, by rescaling the tubular neighbourhoods, one can realise $N(M) \subset N(N)$. Hence for appropriate choice of open sets in the spaces of compactly sections of the tubular neighbourhoods, we have $\tilde{U}_{d} \subset \tilde{U}_{k}$, where we interpret an element of $\tilde{U}_{d}$, which is an exponentiated section of $M$, by what it does to $N \subset M$ instead 1

Hence we have

$$
(M, N) \in \tilde{U}_{d} \times \tilde{U}_{d} \cap \Psi_{k<d}\left(\mathbb{R}^{n}\right) \subset \tilde{U}_{d} \times \tilde{U}_{k} \cap \Psi_{k<d}\left(\mathbb{R}^{n}\right) \subset V_{d} \times V_{k} \cap \Psi_{k<d}\left(\mathbb{R}^{n}\right) .
$$

We have

$$
M \in F_{d}\left(\tilde{U}_{d} \times \tilde{U}_{d} \cap \Psi_{k<d}\left(\mathbb{R}^{n}\right)\right)=\tilde{U}_{d}:=U
$$

which is open in $\Psi_{d}\left(\mathbb{R}^{n}\right)$.

\footnotetext{
${ }^{1}$ Note that $\tilde{U}_{d}$ is not necessarily open as a subset of $\Psi_{k}\left(\mathbb{R}^{n}\right)$.
} 
Definition 4.16. Let $\pi: E_{k<d} \rightarrow G r_{d}\left(\mathbb{R}^{n}\right)$ be the fiber bundle

$$
T h\left(\gamma_{k, d}^{\perp} \rightarrow G r_{k}(P)\right) \longrightarrow E_{k<d} \stackrel{\pi}{\longrightarrow} G r_{d}\left(\mathbb{R}^{n}\right),
$$

where $P \in G r_{d}\left(\mathbb{R}^{n}\right)$ is the base element over which the fiber is given. The fibers are homeomorphic to the Thom space of the anti-canonical vector bundle $\gamma_{k, d}^{\perp}$ over the finite Grassmannian $G r_{k}\left(\mathbb{R}^{d}\right)$.

The projection $\pi$ has a canonical global section given by $P \mapsto(P, \infty)$, where we take the point at infinity of the Thom space in every fiber. Furthermore by including the zero-section into every fiber, we obtain an inclusion into $E$ of an object we will refer to as $G r_{k<d}\left(\mathbb{R}^{n}\right)$, the Grassmannian of $d$-planes in $\mathbb{R}^{n}$ with a chosen $k$-dimensional subplane.

Consider the pullback under $\pi$ of the anti-canonical bundle $\gamma \frac{\perp}{d, n} \rightarrow G r_{d}\left(\mathbb{R}^{n}\right)$ and let

$$
T_{k<d}\left(\mathbb{R}^{n}\right):=T h\left(\pi^{*}\left(\gamma_{d, n}^{\perp}\right) \rightarrow E_{k<d}\right)
$$

An element of $T_{k<d}\left(\mathbb{R}^{n}\right)$ is either

- the basepoint $\infty$, which can be thought of as the empty set (Figure 4.2a), or

- of the form $(e, v)$, where $e \in E_{k<d}$ is given by $e=(P, f), P \in G r_{d}\left(\mathbb{R}^{n}\right)$ with $f$ one of the options below, and $v$ is a vector in $\mathbb{R}^{n}$ perpendicular to $P$.

○ $f=\infty$, in which case we have an element of the form

$$
((P, \infty), v)
$$




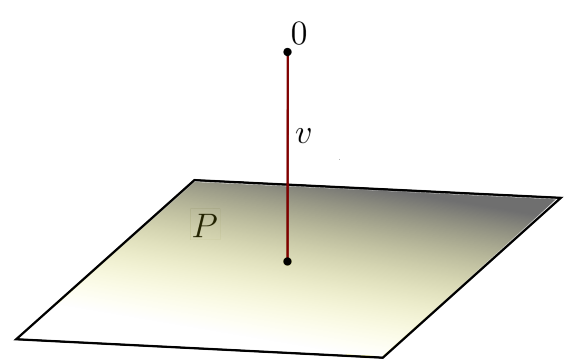

(a) $\varnothing$

(b) $((P, \infty), v)$

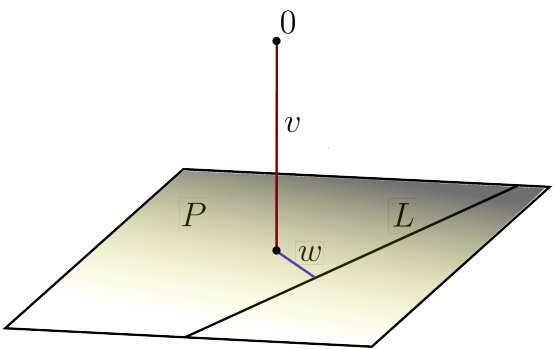

(c) $((P,(L, w)), v)$

Figure 4.2: Examples of elements of $T_{k<d}\left(\mathbb{R}^{n}\right)$.

This can be thought of as a $d$-dimensional plane $P$ inside $\mathbb{R}^{n}$ offset from the origin by a vector $v$ perpendicular to it. This is illustrated in Figure $4.2 \mathrm{~b}$,

$\circ f=(L, w)$ with $L \in G r_{k}(P)$ and $w$ a vector in $P$ perpendicular to $L$, and $v$ is a vector in $\mathbb{R}^{n}$ perpendicular to $P$. Then the element is of the form

$$
((P,(L, w)), v)
$$

and can be thought of as a $d$-dimensional plane $P$ inside $\mathbb{R}^{n}$ offset by a vector $v$, endowed with a $k$-dimensional subplane $L$ that is offset by a normal vector $w$ in $P$ away from $v \in P$. This is illustrated in Figure 4.2c,

\subsubsection{Homotopy type of the space of once nested man- ifolds}

The rest of this section is devoted to proving that $\Psi_{k<d}\left(\mathbb{R}^{n}\right)$ has the homotopy type of $T_{k<d}\left(\mathbb{R}^{n}\right)$. 
Theorem 4.17. There is a weak homotopy equivalence

$$
\Psi_{k<d}\left(\mathbb{R}^{n}\right) \simeq T_{k<d}\left(\mathbb{R}^{n}\right)
$$

Let $\Psi_{k<d}^{0} \subset \Psi_{k<d}\left(\mathbb{R}^{n}\right)$ be given by the pairs of manifolds $(M, N)$ such that $0 \in M$, and furthermore let $\Psi_{k<d}^{00} \subset \Psi_{k<d}^{0}$ be given by the pairs $(M, N)$ such that $0 \in N$.

There is an injective map $q: T_{k<d}\left(\mathbb{R}^{n}\right) \hookrightarrow \Psi_{k<d}\left(\mathbb{R}^{n}\right)$ defined by

$$
\begin{gathered}
q(\infty)=\varnothing ; \\
q((P, \infty), v)=(P-v, \varnothing),
\end{gathered}
$$

by which we mean $P$ realised as a plane in $\mathbb{R}^{n}$ shifted by $v$ away from the origin together with the empty subplane, and generically

$$
q((P,(L, w)), v)=(P-v, L-v-w),
$$

realising $P$ as an affine hyperplane in $\mathbb{R}^{n}$ shifted by $v$, with $L$ as an affine subplane within $P$ shifted by a vector $w \in P$ away from $v$. Note that restricted to the zero-section $E_{k<d} \subset T_{k<d}\left(\mathbb{R}^{n}\right), q$ lands in $\Psi_{k<d}^{0}$. Restricted furthermore to $G r_{k<d}\left(\mathbb{R}^{n}\right) \subset E, q$ lands in $\Psi_{k<d}^{00}$. Although we will not need this fact in the proof, it is interesting to point out that $q\left(G r_{k<d}\left(\mathbb{R}^{n}\right)\right)$ sits inside $\Psi_{k<d}^{00}$ as a deformation retract, with retraction given by radial expansion. 
Proposition 4.18. There is a weak homotopy equivalence

$$
\Psi_{k<d}^{0} \simeq E_{k<d}
$$

Proof. Consider the projection $\Psi_{k<d}^{0} \rightarrow G r_{d}\left(\mathbb{R}^{n}\right)$ given by sending $(M, N)$ to the tangent space $T_{0} M$ of $M$ at the origin. This map is a fibration with fiber $\Psi_{k<d}^{0, P}$ given by the pairs of manifolds $(M, N)$ in $\Psi_{k<d}^{0}$ such that $T_{0} M=P \in$ $G r_{d}\left(\mathbb{R}^{n}\right)$. Define $\Psi_{k}\left(P \subset \mathbb{R}^{n}\right)$ to be the elements of $\Psi_{k<d}^{0, P}$ for which $M=P$, so the space of submanifolds of $P$ considered as a subspace of $\mathbb{R}^{n}$.

We have the following diagram, the left triangle of which is illustrated in Figure 4.3.

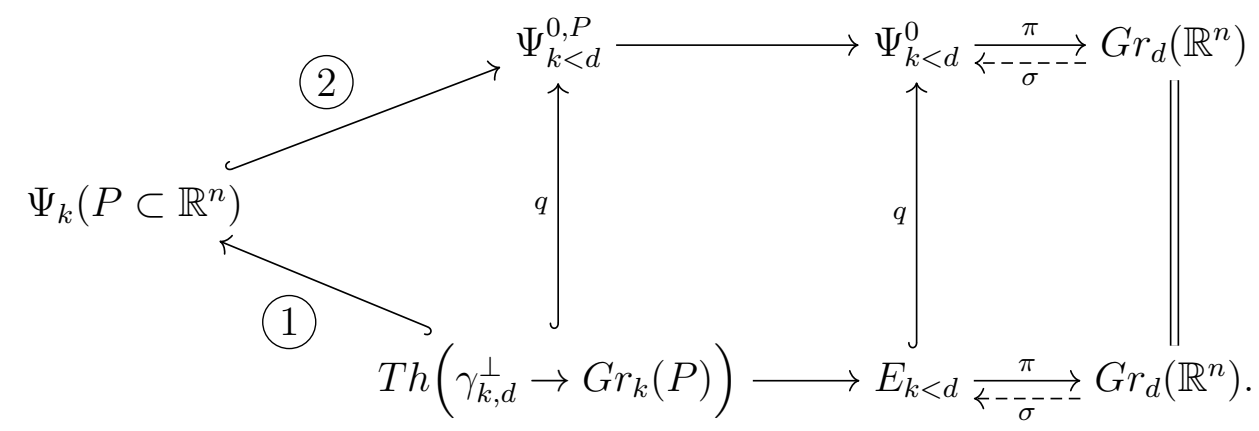

All upward arrows can be thought of as inclusions where some of the manifolds are linear, and hence this diagram commutes. We have that the map (1) is a homotopy equivalence by the non-nested version of Theorem 4.12 proved in [GRW10] (Theorem 4.12 in the previous section).

We will now show that the map (2) includes $\Psi_{k}\left(P \subset \mathbb{R}^{n}\right)$ into $\Psi_{k<d}^{0, P}$ as a deformation retract. We precompose the function $\eta: \Psi_{d}^{0} \rightarrow(0, \infty)$ from 


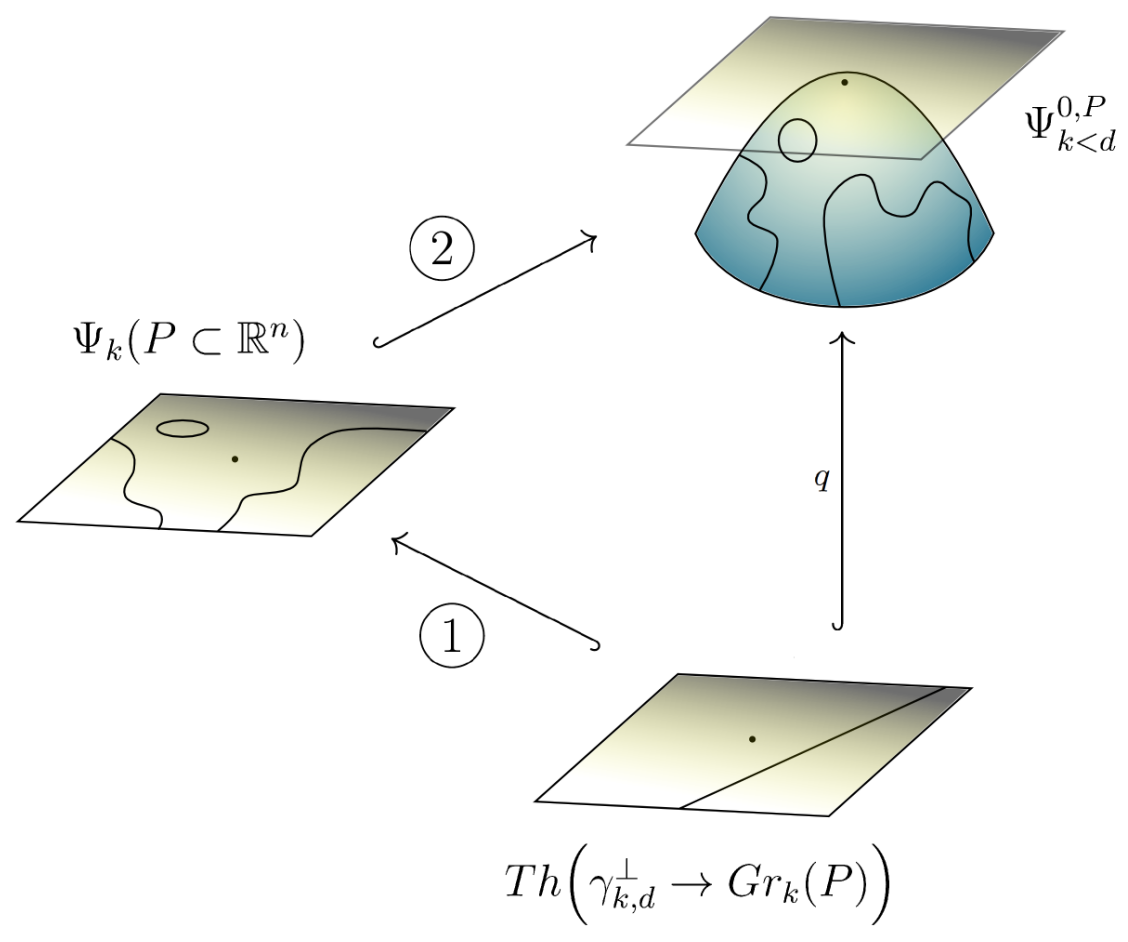

Figure 4.3: An illustration of typical elements of the spaces of manifolds $\Psi_{k<d}^{0, P}$ and $\Psi_{k}\left(P \subset \mathbb{R}^{n}\right)$, and of $T h\left(\gamma_{k, d}^{\perp} \rightarrow G r_{k}(P)\right)$, viewed as a space of affine linear $k$-manifolds in $P^{d}$. 
Lemma 4.13 with the forgetful map $\Psi_{k<d}^{0} \rightarrow \Psi_{d}^{0}$, calling the resulting continuous map again $\eta$. We have that all $(M, N) \in \Psi_{k<d}^{0, P}$ have the property that $\exp : T_{0} M=P \rightarrow M$ is a diffeomorphism within the ball of radius $\eta(M)$. Moreover, we can interpolate between the manifold and its tangent space viewed as a subspace of $\mathbb{R}^{n}$ using the rays described in condition (C2).

We want to define a homotopy $F: \Psi_{k<d}^{0, P} \times[0,1] \rightarrow \Psi_{k<d}^{0, P}$ with the property that

$$
\begin{gathered}
F((M, N), 0)=(M, N), \\
F((M, N), 1) \in \Psi_{k}\left(P \subset \mathbb{R}^{n}\right),
\end{gathered}
$$

and

$$
F((P, N), 1)=(P, N)
$$

To do this we will apply a combination of the following two continuous procedures. We will flatten $M$ in a ball of radius $\eta / 2$ around 0 to match its tangent space, using the annulus between $\eta / 2$ and $\eta$ to interpolate, while in the process lifting up the submanifold $N$ to a submanifold of $T_{0} M^{\eta / 2}=T_{0} M \cap B_{\eta / 2}(0)$ (Lifting). At the same time we will expand the ball of radius $\eta / 2$ around 0 in $\mathbb{R}^{n}$ to increasingly fill all of $\mathbb{R}^{n}$ (Expanding).

\section{Lifting}

By Lemma 4.13, we have that inside the ball of radius $\eta$, the map $\exp _{M}$ : $T_{0} M \rightarrow M$ is injective, and moreover for a fixed $x \in T_{0} M^{\eta}$ the ray $s x+(1-$ $s) \exp _{M}(x)$, for $s \in[0,1]$, intersects $M$ only in $\exp (x)$, and that $B_{\eta}(0) \cap M \cong$ $\mathbb{R}^{d}$. 


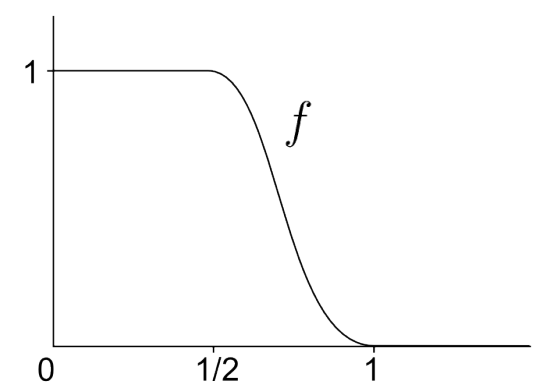

Figure 4.4: Example of a smooth interpolating function $f$.

Let $f: \mathbb{R}_{\geq 0} \rightarrow \mathbb{R}_{\geq 0}$ be a smooth map with the properties that it equals 1 on the interval $[0,1 / 2]$, it is monotonically decreasing on the interval $(1 / 2,1)$ and it is 0 on $[1, \infty)$, as shown in figure 4.4 .

For each $(M, N) \in \Psi_{k<d}^{0, P}$, we will define a diffeomorphism $\psi_{M, t}: \mathbb{R}^{n} \rightarrow \mathbb{R}^{n}$ in the following way. Consider all the points on these rays, i.e.

$$
R:=\left\{s x+(1-s) \exp _{M}(x) \mid x \in T_{0} M^{\eta} ; s \in[0,1]\right\}
$$

We first define the action of $\psi_{M, t}$ on $R$. Let $\rho=t f(\|x\|)$ for $f$ as above and $t \in[0,1)$, and define

$$
\psi_{M, t}\left(s x+(1-s) \exp _{M}(x)\right):=s x+(1-s)\left(\rho x+(1-\rho) \exp _{M}(x)\right) .
$$

In words, $\psi_{M, t}$ has the effect on $R$ that it shrinks the ray $s x+(1-s) \exp _{M}(x)$ by a factor of $t f(\|x\|)$ while fixing $x$, as illustrated in figure. We have that $\psi_{M, t}(R) \subset R$. To define what $\psi_{M, t}$ does to points outside of $R$, we pick a small open neighbourhood $D(R)$ of $R$. Outside of $D(R), \psi_{M, t}$ is the identity map. On $D(R)$, we interpolate between the action on $R$ and the identity map, which can be done because 'shrinking' is homotopic to the identity. We can choose 
$D(R)$ and $\left.\psi_{M, t}\right|_{D(R)}$ in such a way that the only points of $M$ that are moved by $\psi_{M, t}$ are contained in $R$. Note that for $t=0, \psi_{M, t}$ is the identity map on $\mathbb{R}^{n} \cdot 2$

We now define a map $L: \Psi_{k<d}^{0, P} \times[0,1) \rightarrow \Psi_{k<d}^{0, P}$ as follows:

$$
L((M, N), t)=\psi_{M, t} \circ(M, N)
$$

Here by $\psi_{M, t} \circ(M, N)$ we mean the subsets $\left(M^{\prime}, N^{\prime}\right)$ of $\mathbb{R}^{n}$ that are the image of $(M, N)$ under applying $\psi_{M, t}$ to $\mathbb{R}^{n}$ (alternatively, they are pulled back with respect to the diffeomorphism $\left.\psi_{M, t}^{-1}\right)$. As $t \rightarrow 1, L_{t}$ approaches a map that flattens $M$, and $N$ inside of it, in a disc of radius $\eta / 2$ such that it matches $T_{0} M=P$. In the annulus between $\eta / 2$ and $\eta$, the new pair of manifolds interpolate smoothly between the flattened disc and the rest of $(M, N)$, which is unchanged outside the ball of radius $\eta$. This is illustrated in Figure 4.5a and $4.5 \mathrm{~b}$.

The function $\eta$ varies continuously with respect to varying $M$. For fixed $x \in T_{0} M=P$, the points $\exp _{M}(x)$ also vary continuously with respect to changing $M$. Hence the manifolds $\psi_{M, t} \circ(M, N)$ vary continously with respect to changing $\Psi_{k<d}^{0, P}$ as well as $t$, hence $L$ is a continous map.

\section{Expanding}

For $\alpha \in(0, \infty]$, define the smooth family of diffeomorphisms $\phi_{\alpha}$ from $\mathbb{R}^{n}$ to $B_{\alpha}(0)$ given by:

\footnotetext{
${ }^{2}$ Note that we have not shown that $\psi_{M, t}$ is a smooth family of diffeomorphisms with respect to varying $(M, N) \in \Psi_{k<d}^{0, P}$, which would require a consistent choice of $D(R)$.
} 


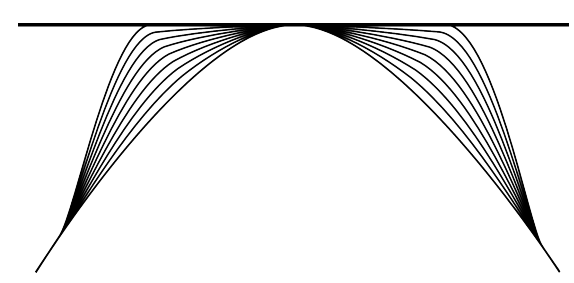

(a) Lifting the manifold.

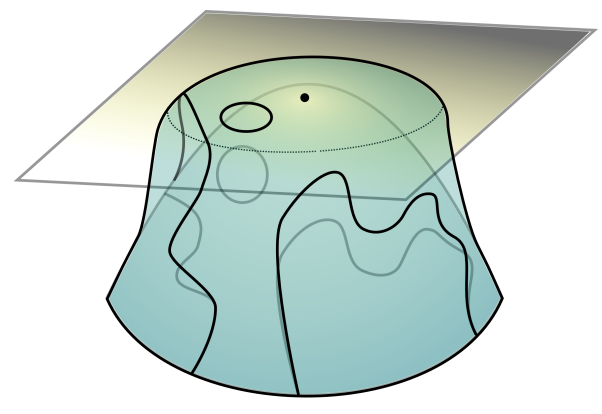

(b) Lifting the manifold.

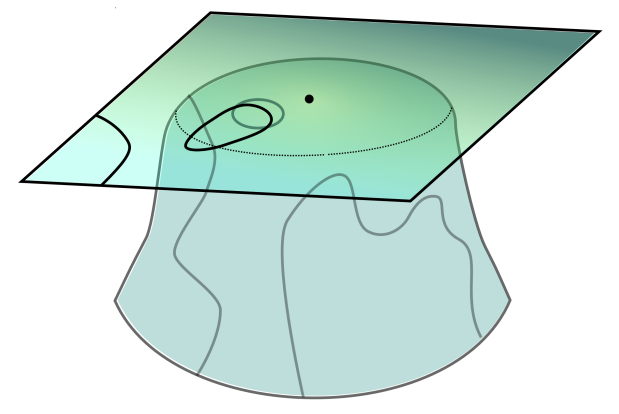

(c) Expanding the manifold.

Figure 4.5: This figure illustrates the lifting and expanding procedures. We flatten the manifold in a disc around 0 to match its tangent space (lifting) and subsequently expand the disc such that it fills the plane, while keeping finite distances finite (expanding). 


$$
\phi_{\alpha}(x)= \begin{cases}0 & x=0 \\ \alpha \arctan \left(\frac{\|x\|}{\alpha}\right) \frac{x}{\|x\|} & x>0 ; \alpha<\infty \\ x & \alpha=\infty .\end{cases}
$$

In the following we will set $\alpha=\eta / 2 t$, where $\eta: \Psi_{k<d}^{0, P} \rightarrow(0, \infty]$ is the map from Lemma 4.13 precomposed with the forgetful map $\Psi_{k<d}^{0, P} \rightarrow \Psi_{d}^{0}$. Now we can define a map $E: \Psi_{k<d}^{0, P} \times[0,1] \rightarrow \Psi_{k<d}^{0, P}$ as follows:

$$
E((M, N), t)=\phi_{\eta(M) / 2 t}^{-1}(M, N)
$$

The map $E$ is continuous because the map $\eta$ and therefore the family of diffeomorphisms $\phi_{\eta(M) / t}^{-1}$ is continuous with respect to varying $(M, N)$, and they also depend continuously on $t$.

We will combine the lifting and expanding procedures in the deformation retraction $F: \Psi_{k<d}^{0, P} \times[0,1] \rightarrow \Psi_{k<d}^{0, P}$ :

$$
F((M, N), t)= \begin{cases}E_{t} \circ L_{t}((M, N)) & 0 \leq t<1 \\ \left(P, N^{\prime}\right) & t=1 .\end{cases}
$$

Where $\left(P, N^{\prime}\right) \in \Psi_{k}\left(P \subset \mathbb{R}^{n}\right)$, with $N^{\prime}$ given by $\phi_{\eta(M) / 2}^{-1}\left(\exp _{B_{\eta / 2}^{M}(0)}^{-1}(N)\right)$ : the submanifold $N$ lifted to the tangent space of $M$ at 0 within an $\eta(M) / 2$ disc, which is subsequently expanded to fill the plane $P$. If $M$ was $P$ to start with, then by construction $\eta(P)=\infty$, hence both the tangential exponential map at 0 and $\phi_{\eta(M) / 2 t}$ act as the identity, hence $F((P, N), t)=(P, N)$ for all $t$. This is illustrated in Figure $4.5 \mathrm{c}$. 
Hence we conclude that the map (2) is the inclusion of $\Psi_{k}\left(P \subset \mathbb{R}^{n}\right)$ as a deformation retract of $\Psi_{k<d}^{0, P}$.

Between the top and bottom line of diagram 4.6 we now have a map of fibrations, of which the outermost maps are homotopy equivalences. Applying the five lemma to the long exact sequence in homotopy yields that the map in the middle is a weak homotopy equivalence, as required.

Proof of Theorem 4.17. $\Psi_{k<d}^{0}$ supports a vector bundle $\nu_{0}$ of dimension $n-d$ which is given over a point $(M, N)$ by taking the normal bundle of $M$ at 0 as a subspace of $\mathbb{R}^{n}$. On $q\left(E_{k<d}\right) \subset \Psi_{k<d}^{0}$, this normal bundle restricts to $q_{*}\left(\pi^{*}\left(\gamma_{d, n}^{\perp}\right) \rightarrow E_{k<d}\right)$.

There is a continuous map $e_{k<d}: \nu_{0} \rightarrow \Psi_{k<d}\left(\mathbb{R}^{n}\right)$ given by

$$
((M, N), v) \mapsto(M+v, N+v)
$$

the translate of $(M, N)$ by $v$. This map is injective on $D_{\varepsilon \circ F_{d}}\left(\nu_{0}\right)$, where $\varepsilon$ is the map on $\Psi_{d}\left(\mathbb{R}^{n}\right)$ defined in Lemma 4.10 and $F_{d}: \Psi_{k<d}\left(\mathbb{R}^{n}\right) \rightarrow \Psi_{d}\left(\mathbb{R}^{n}\right)$ is the forgetful map. We have the commuting diagram

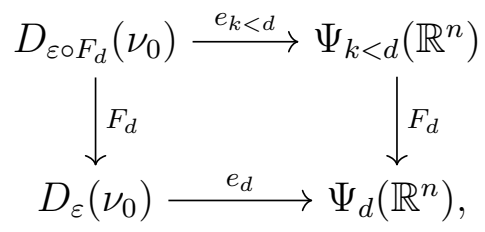

where $e_{d}=e$ is the map used in the proof of Theorem 4.12. Both $F$ and $e_{d}$ are continuous open maps, hence it follows that $e_{k<d}$ is an open map.

We proceed analogously as in the proof of Theorem 4.12, Let $C$ be the com- 
plement in $\Psi_{k<d}\left(\mathbb{R}^{n}\right)$ of $e_{k<d}\left(\bar{D}_{\varepsilon \circ F_{d}} \nu_{0}\right)$. By applying the homotopy equivalence between $S^{n-1}$ and $D^{n} \backslash\{0\}$ fiberwise, we have that

$$
\partial e_{k<d}\left(\bar{D}_{\varepsilon \circ F_{d}} \nu_{0}\right) \simeq e_{k<d}\left(\bar{D}_{\varepsilon \circ F_{d}} \nu_{0}\right) \backslash \Psi_{k<d}^{0}
$$

hence $C \simeq C^{\prime}=\Psi_{k<d}\left(\mathbb{R}^{n}\right) \backslash \Psi_{k<d}^{0} . C^{\prime}$ is the set of pairs of manifolds $(M, N)$ such that $M$ does not contain the origin. Hence $C^{\prime}$ can be contracted to $\varnothing$ by the homotopy $F^{\prime}: C^{\prime} \times[0,1] \rightarrow C^{\prime}$ given by:

$$
F^{\prime}((M, N), t)= \begin{cases}T_{t}((M, N)) & 0 \leq t<1 \\ \varnothing & t=1 .\end{cases}
$$

We have that $C \subset V$ where $V$ is the complement of $e_{k<d}\left(\bar{D}_{\varepsilon \circ F_{d} / 2} \nu_{0}\right)$, which is open as $e_{k<d}$ is an open map. Applying the homotopy equivalence between the annulus and the circle fiberwise, we see that $C$ is a neighbourhood deformation retract of $V$. Hence collapsing $C$ in $\Psi_{k<d}\left(\mathbb{R}^{n}\right)$ yields a homotopy equivalent space, which is homeomorphic to the space obtained by collapsing the sphere bundle $S_{\varepsilon \circ F_{d}} \nu_{0}$, i.e. the Thom space of $\nu_{0} \rightarrow \Psi_{k<d}^{0}$. This Thom space is homotopy equivalent to $\operatorname{Th}\left(\pi^{*}\left(\gamma_{d, n}^{\perp}\right) \rightarrow E_{k<d}\right)=T_{k<d}\left(\mathbb{R}^{n}\right)$. 


\subsection{Homotopy type of spaces of arbitrarily nested manifolds}

\subsubsection{Definitions}

Definition 4.19. Let $I$ be the list $k_{1}<k_{2}<\ldots<k_{m}$, with $k_{1} \geq 0$ and $k_{m}<n$. We define $\Psi_{I}\left(\mathbb{R}^{n}\right)$ to be the set of $m$-tuples of subsets

$$
M_{I}=\left(M_{m}, M_{m-1}, \ldots, M_{1}\right),
$$

of $\mathbb{R}^{n}$, that are nested in the sense that $M_{m} \supset \ldots \supset M_{2} \supset M_{1}$, and for each $i$, $M_{i}$ is a smooth $k_{i}$-dimensional submanifold of $\mathbb{R}^{n}$ without boundary and closed as a subset of $\mathbb{R}^{n}$. Note in particular that if the submanifold of dimension $k_{i}$ is the empty set, then so are the manifolds of lower dimensions. This set pointwise includes into the set $\Psi_{k_{1}}\left(\mathbb{R}^{n}\right) \times \ldots \times \Psi_{k_{m}}\left(\mathbb{R}^{n}\right)$. We endow $\Psi_{I}\left(\mathbb{R}^{n}\right)$ with the subspace topology from its inclusion in this product.

Definition 4.20. Let $I$ be the list $k_{1}<k_{2}<\ldots<k_{m}$, for $k_{1} \geq 0$. We define $T_{I}\left(\mathbb{R}^{n}\right)$ by induction on $m$, the length of the ordered list $I$. The base case for a list of length 2 is given in Definition 4.16 . Suppose we have defined $T_{J}\left(\mathbb{R}^{n^{\prime}}\right)$ for lists $J$ of length less than $m$ and any $n^{\prime}$ such that $J<n^{\prime}$. In particular we have defined $T_{k_{1}<k_{2}<\ldots<k_{m-1}}\left(\mathbb{R}^{k_{m}}\right):=T_{I^{\prime}}\left(\mathbb{R}^{k_{m}}\right)$. Consider then the fibration

$$
T_{I^{\prime}}\left(P^{k_{m}}\right) \longrightarrow E_{I^{\prime}<d} \stackrel{\pi}{\longrightarrow} G r_{k_{m}}\left(\mathbb{R}^{n}\right),
$$

where $P^{k_{m}} \in G r_{k_{m}}\left(\mathbb{R}^{n}\right)$ is the base element over which the fiber is given. 
Define

$$
T_{I}\left(\mathbb{R}^{n}\right)=T_{I^{\prime}<k_{m}}\left(\mathbb{R}^{n}\right):=\operatorname{Th}\left(\pi^{*}\left(\gamma_{k_{m}, n}^{\perp}\right) \rightarrow E_{I^{\prime}<d}\right)
$$

An element of $T_{I}\left(\mathbb{R}^{n}\right)$ is either $\infty$ or of the form $(P, L, v)$, for $P \in G r_{d}\left(\mathbb{R}^{n}\right)$, $L \in T_{I^{\prime}}(P)$ and $v \in \gamma_{d, n}^{\perp}(P)$, a vector normal to $P$ in $\mathbb{R}^{n}$.

\subsubsection{Homotopy type of spaces of nested manifolds}

The rest of this section is devoted to proving that $\Psi_{I}\left(\mathbb{R}^{n}\right)$ has the homotopy type of $T_{I}\left(\mathbb{R}^{n}\right)$.

Theorem 4.21. There is a weak homotopy equivalence

$$
\Psi_{I}\left(\mathbb{R}^{n}\right) \simeq T_{I}\left(\mathbb{R}^{n}\right)
$$

We will proceed by induction of the length of the list $I$. The base case for $|I|=2$ was shown in Theorem 4.17 Now let

$$
I=I^{\prime}<d
$$

and assume that we have shown $\Psi_{I^{\prime}}\left(\mathbb{R}^{n}\right) \simeq T_{I^{\prime}}\left(\mathbb{R}^{n}\right)$ for all $n>I^{\prime}$.

We can inductively define an injective map $q: T_{I}\left(\mathbb{R}^{n}\right) \hookrightarrow \Psi_{I}\left(\mathbb{R}^{n}\right)$ realising an element in $T_{I}\left(\mathbb{R}^{n}\right)$ as a nested sequence of off-set planes. Specifically, suppose we have defined the map $q: T_{I^{\prime}}\left(\mathbb{R}^{d}\right) \hookrightarrow \Psi_{I^{\prime}}\left(\mathbb{R}^{d}\right)$. An element of $T_{I}\left(\mathbb{R}^{n}\right)$ is either $\infty$ or of the form $\left(P, L_{I^{\prime}}, v\right)$, for $P \in G r_{d}\left(\mathbb{R}^{n}\right), L_{I^{\prime}} \in T_{I^{\prime}}(P)$ and $v \in \gamma_{d, n}^{\perp}(P)$, a

\footnotetext{
${ }^{3}$ The $|I|=2$ case was separated out for clarity, we could also have started the induction with $|I|=1$, the non-nested case.
} 
normal vector to $P$ in $\mathbb{R}^{n}$. We define

$$
q(\infty)=\varnothing
$$

and

$$
q\left(P, L_{I^{\prime}}, v\right)=\left(P-v, q\left(L_{I^{\prime}}\right)\right)
$$

the shifted plane $P-v$ together with the $I^{\prime}$-nested manifold $q\left(L_{I^{\prime}}\right) \in \Psi_{I^{\prime}}(P-$ $\left.v \subset \mathbb{R}^{n}\right) \cong \Psi_{I^{\prime}}\left(\mathbb{R}^{d}\right)$.

Let $\Psi_{I}^{0} \subset \Psi_{I}\left(\mathbb{R}^{n}\right)$ be given by the lists of manifolds such that $0 \in M^{d}$, i.e. the origin is contained in the top dimensional manifold. The map $q$ restricted to the base $E_{I}$ lands in $\Psi_{I}^{0}$.

Proposition 4.22. There is a weak homotopy equivalence

$$
\Psi_{I}^{0} \simeq E_{I}
$$

Proof. This proof will proceed along similar lines as the proof of Proposition 4.18. Consider the projection $\Psi_{I}^{0} \rightarrow G r_{d}\left(\mathbb{R}^{n}\right)$ given by sending $M_{I}$ to $T_{0} M^{d}$, the tangent space at the origin of the top dimensional manifold. This map is a fibration with fiber $\Psi_{I}^{0, P}$ given by sequences of manifolds $M_{I}$ such that $T_{0} M^{d}=P \in G r_{d}\left(\mathbb{R}^{n}\right)$. We have the following commuting diagram: 


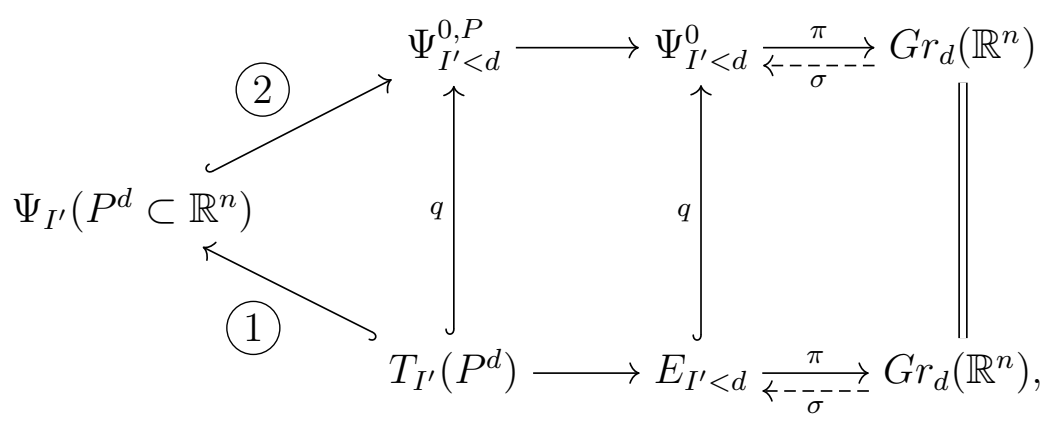

where $\Psi_{I^{\prime}}\left(P^{d} \subset \mathbb{R}^{n}\right)$ the space of $I^{\prime}$-nested submanifolds of $P \cong \mathbb{R}^{d}$. The map (1) is a weak homotopy equivalence by the induction hypothesis. We will now show that the map 2 is the inclusion of a deformation retract by applying the same procedure of lifting and expanding as in the proof of Proposition 4.18. For either case, we precompose the map $\eta$ constructed in Lemma 4.13 with the forgetful map $\Psi_{I}\left(\mathbb{R}^{n}\right) \rightarrow \Psi_{d}\left(\mathbb{R}^{n}\right)$.

\section{Lifting}

Define $\psi_{M, t}: \mathbb{R}^{n} \rightarrow \mathbb{R}^{n}$ as before. We now define a map $L: \Psi_{I}^{0, P} \times[0,1) \rightarrow \Psi_{I}^{0, P}$ as follows:

$$
L\left(M_{I}, t\right)=\psi_{M, t} \circ M_{I}
$$

As $t \rightarrow 1, L_{t}$ approaches a map that flattens the top dimensional manifold $M^{d}$, and with it the $I^{\prime}$-nested submanifold inside of it, in a disc of radius $\eta / 2$ such that it matches $T_{0} M^{d}=P$. In the annulus between $\eta / 2$ and $\eta$, the new pair of manifolds interpolate smoothly between the flattened disc and the rest of $M_{I}$, which is unchanged outside the ball of radius $\eta$. By the same arguments as before, $L$ is continuous. 


\section{Expanding}

Define the smooth family of diffeomorphisms $\phi_{\alpha}$ from $\mathbb{R}^{n}$ to $B_{\alpha}(0)$ as before. We can then define a map $E: \Psi_{I}^{0, P} \times[0,1] \rightarrow \Psi_{I}^{0, P}$ as follows:

$$
E\left(M_{I}, t\right)=\phi_{\eta(M) / 2 t}^{-1} M_{I}
$$

As before this map is continuous.

We will combine the lifting and expanding procedures in the deformation retraction $F: \Psi_{I}^{0, P} \times[0,1] \rightarrow \Psi_{I}^{0, P}:$

$$
F\left(M_{I}, t\right)= \begin{cases}E_{t} \circ L_{t}\left(M_{I}\right) & 0 \leq t<1 \\ \left(P, N^{\prime}\right) & t=1 .\end{cases}
$$

Where $\left(P, N_{I^{\prime}}^{\prime}\right) \in \Psi_{I^{\prime}}\left(P \subset \mathbb{R}^{n}\right)$, with $N_{I^{\prime}}^{\prime}$ given by $\phi_{\eta(M) / 2}^{-1}\left(\exp _{B_{\eta / 2}^{M}(0)}^{-1}\left(N_{I^{\prime}}\right)\right)$ : the nested submanifold $N_{I^{\prime}} \subset M_{I}$ lifted to the tangent space of $M^{d}$ at 0 within an $\eta(M) / 2$ disc, which is subsequently expanded to fill the plane $P^{d}$. If $M$ was $P$ to start with, then by construction $\eta(P)=\infty$, hence both the tangential exponential map at 0 and $\phi_{\eta(M) / 2 t}$ act as the identity, hence $F\left(\left(P, N_{I^{\prime}}\right), t\right)=$ $\left(P, N_{I^{\prime}}\right)$ for all $t$.

Hence $\Psi_{I^{\prime}}\left(P^{d} \subset \mathbb{R}^{n}\right)$ is a deformation retract of $\Psi_{I^{\prime}<d}^{0, P}$. It follows that the vertical maps in diagram 4.9 again form a map of fibrations with the outer maps homotopy equivalences, hence the middle vertical map $q: \Psi_{I}\left(\mathbb{R}^{n}\right) \rightarrow$ $T_{I}\left(\mathbb{R}^{n}\right)$ is a weak homotopy equivalence.

Proof of Theorem 4.21. This proof proceeds analogously to the proof of The- 
orem 4.17.

Let $I=I^{\prime}<d$. The space $\Psi_{I}^{0}$ supports a vector bundle $\nu_{0}$ of dimension $n-d$ which is given over a point $M_{I}$ by taking the normal bundle of the top dimensional manifold $M=M^{d}$ at 0 as a subspace of $\mathbb{R}^{n}$. On $q\left(E_{I}\right) \subset \Psi_{I}^{0}$, this normal bundle restricts to $q_{*}\left(\pi^{*}\left(\gamma_{d, n}^{\perp}\right) \rightarrow E_{I}\right)$.

There is a continuous map $e_{I}: \nu_{0} \rightarrow \Psi_{I}\left(\mathbb{R}^{n}\right)$ given by

$$
\left(M_{I}, v\right) \mapsto M_{I}+v
$$

the translate of all manifolds $M_{I}$ by $v$. This map is injective on $D_{\varepsilon \circ F_{d}}\left(\nu_{0}\right)$, where $\varepsilon$ is the map on $\Psi_{d}\left(\mathbb{R}^{n}\right)$ defined in Lemma 4.10 and $F_{d}: \Psi_{I}\left(\mathbb{R}^{n}\right) \rightarrow$ $\Psi_{d}\left(\mathbb{R}^{n}\right)$ is the forgetful map. We have the commuting diagram

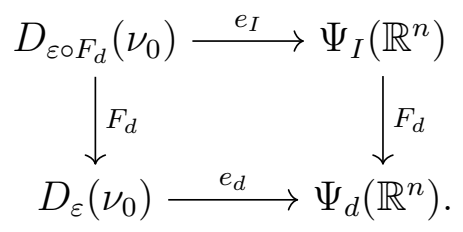

For $e_{d}=e$ the map used in the proof of Theorem 4.12. Both $F$ and $e_{d}$ are continuous open maps, hence it follows that $e_{I}$ is an open map.

Let $C$ be the complement in $\Psi_{I}\left(\mathbb{R}^{n}\right)$ of $e_{I}\left(\bar{D}_{\varepsilon \circ F_{d}} \nu_{0}\right)$. By applying the homotopy equivalence between $S^{n-1}$ and $D^{n} \backslash\{0\}$ fiberwise, we have that

$$
\partial e_{I}\left(\bar{D}_{\varepsilon \circ F_{d}} \nu_{0}\right) \simeq e_{I}\left(\bar{D}_{\varepsilon \circ F_{d}} \nu_{0}\right) \backslash \Psi_{I}^{0}
$$

hence $C \simeq C^{\prime}=\Psi_{I}\left(\mathbb{R}^{n}\right) \backslash \Psi_{I}^{0}$.

$C^{\prime}$ is the set of nested manifolds $M_{I}$ such that the top dimensional manifold $M$ does not contain the origin. Hence $C^{\prime}$ can be contracted to $\varnothing$ by the homotopy 
$F^{\prime}: C^{\prime} \times[0,1] \rightarrow C^{\prime}$ given by:

$$
F^{\prime}\left(M_{I}, t\right)= \begin{cases}T_{t}\left(M_{I}\right) & 0 \leq t<1 \\ \varnothing & t=1 .\end{cases}
$$

We have that $C \subset V$ where $V$ is the complement of $e_{I}\left(\bar{D}_{\varepsilon \circ F_{d} / 2} \nu_{0}\right)$, which is open as $e_{I}$ is an open map. Applying the homotopy equivalence between the annulus and the circle fiberwise, we see that $C$ is a neighbourhood deformation retract of $V$. Hence collapsing $C$ in $\Psi_{I}\left(\mathbb{R}^{n}\right)$ yields a homotopy equivalent space, which is homeomorphic to the space obtained by collapsing the sphere bundle $S_{\varepsilon \circ F_{d}} \nu_{0}$, i.e. the Thom space of $\nu_{0} \rightarrow \Psi_{I}^{0}$. This Thom space is homotopy equivalent to $T h\left(\pi^{*}\left(\gamma_{d, n}^{\perp}\right) \rightarrow E_{I}\right)=T_{I}\left(\mathbb{R}^{n}\right)$. 


\section{Acknowledgements}

First and foremost I want to thank my supervisor Ulrike Tillmann. I owe her not only the incredible opportunity to pursue this second DPhil in the amazing environment that is the Oxford Mathematics Department, but also the courage to complete the first one at an accelerated pace. Throughout my DPhil she held the space for me to develop mathematically and also as a person, without imposing boundaries but with plenty of encouragement whenever I needed it. Meeting her as my first mathematical role model has made an impact on my life that goes beyond description - I literally have no idea what my life would have been like if I had not met her. I feel deeply honoured that she somehow, somewhere, found an unwavering faith in me, which has been a foundational support throughout the last few years and will stay with me throughout my career.

One cannot be a mathematician in isolation. I have been blessed with the support of a number of researchers in the field that have acted to varying extent as academic mentors in the last few years. In particular I want to thank Chris Douglas, André Henriques, Oscar Randal-Williams, Søren Galatius and Diarmuid Crowley for their support. For inspiring, encouraging and inspirational conversations I furthermore thank Federico Cantero Morán, Andrew Ranicki, 
Nathalie Wahl, Mike Hill, Doug Ravenel, Constantin Teleman, Bruce Bartlett, Mark Powell, Karen Vogtmann and Nathan Perlmutter. I thank Markus Land and Fabian Hebestreit especially for comments on chapter 1 I I thank Masaki Nakagawa for a useful email exchange and Robert Bruner for help with calculations in MAGMA. Chris Schommer-Pries and André Henriques thanks for their comments in my thesis as examiners.

Ian Hewitt I thank for the incredible opportunity to be a lecturer at Trinity College, which has been a substantive and rewarding part of my life over the past three years. Thanks furthermore for his laid back and therapeutic advice as a college advisor. I thank Ian and also Melanie Rupflin and Tom Brown for three years of smooth and fun collaboration, especially around Admission time. I want to thank all of my students at Trinity for making my time as a lecturer such an amazing experience. Furthermore I thank the members of the Trinity SCR for sharing their inspiring research stories with me over the years.

Maarten Kleinhans I thank for being an inspirational figure and great (nonmathematical) collaborator.

I want to thank everyone who makes the Oxford Maths department such an amazing place to be. Particular thanks to Henry Bradford, David Hume, Rob Kropholler and the others who were hanging around the department five years ago for rekindling my mathematical curiosity. I thank Lucas Castello Branco and Matthias Wink for being my loyal office buddies over the years, and a great source of solace and support. I want to thank my academic siblings, of whom there are many, but particularly Tom Zeman, Jan Steinebrunner, 
Luciana Basualdo Bonatto, Simon Gritschacher and Nina Otter. Furthermore I thank Gareth Wilkes, Claudio Llosa Isenrich, Elia Fioravanti, Jo French, Giles Gardam, Ric Wade, Paul Ziegler, Carolina Matté Gregory, Emily Cliff, Adam \& Lena Gal, Mark Penney, Manuel Araújo and many more friends for brightening my time at the Maths department.

Claudia Scheimbauer has been a great friend as well as an amazing source of support academically. Thanks in particular for comments on the thesis.

I thank my friends and colleagues in the field for being such amazing people, particularly Nissim Ranade, Catherine Ray, Manuel Krannich, Jens Reinhold, Sander Kupers, Inbar Klang, Calista Bernard, Nils Prigge and Martin PalmerAnghel. Rachael Boyd is my true buddy in the field. I hope we will continue to see each other lots over the next few years as postdocs.

I thank my dear festival gang, particularly Federico Vigolo, Nici Heuer and Andrea Skiavi. Thanks Alessandro Sisto, my sweet butterfly and good friend. Thanks Rob, Rob, Rob. Chiara Meccariello, thanks for staying with us often, it was always great to have you over. I thank my sweet friends outside of maths, in particular Hongjia Zhang, Anna Grebenchtchikova, Jens van Egmond, Paula Koelemeijer, Matan Shenhav, Wilke van der Schee, Thessa Fokkema and Juho Asikainen.

I thank my cat Raja, shining in pictures from overseas, relentlessly and lazily cheering me up. Thanks to my Springboard group for a very supportive and transformative experience.

I thank the pieces of natural wilderness in Oxford that have nourished me throughout my time in this city. In particular Port Meadow, Wolvercote Com- 
mons, the Trap Grounds and the stretch of canal from Jericho to Wolvercote. I thank the people that have fought to maintain these bits of heaven on earth. I thank the flowers that grow in the walls of ancient buildings in Oxford for reminding me that nature is just there, being, no matter how pompous and driven our intellectual ambitions, no matter how nihilistic and desperate our souls. I also want to thank the community of Wolvercote for embracing me as a villager in what must be the number one place on the planet to live.

I would like to thank my parents Anneke Kerkhof and Dick Hoekzema for their incredible love and support, and their patience while I have been torn away from them by ethereal projects such as a thesis (or two). I thank my sisters Elseline and Tessa and nephews Timo, Florian and Sava and nieces Jasmijn and Lise, whom I all love deeply. I thank Ike van den Berg for being there for us, for being with us, and I thank her most deeply for still being. Jos Wasserman, in our last conversation I had the opportunity to tell you about the very conception of this endeavour - I wish you could have been here to see its completion, and to see how we have grown.

I thank my beloved muse Aura Raulo for falling from the astral plane straight into my life. You kindled in me a new-found fire of unexpected otherworldly joy and inspiration. Together we thank the BED institute for funding of the spiritual kind.

Lastly I am deeply grateful to Thomas Wasserman, my partner in love and science. I thank you for many useful conversations and many more useful cuddles. I thank you for holding my hand tightly while we fought to keep our heads above the surface of the black water, and I thank you for clumsily clambering out with me while using each other as stepping stones. You are 
the language in which my word for joy is written. I love you.

\section{Funding}

For financial support that made this research possible I would like to thank the EPSRC, the Hendrik Mullerfonds, the Fundatie van de Vrijvrouwe van Renswoude, the Oxford Mathematical Institute and Trinity College. 


\section{Bibliography}

[ABS64] M. F. Atiyah, R. Bott, and A. Shapiro. Clifford modules. Topology, 3:3-38, 1964.

[AHR] M. Ando, M. J. Hopkins, and C. Rezk. Multiplicative orientations of KO-theory and the spectrum of topological modular forms. http://www.math.uiuc.edu/ mando/papers/koandtmf.pdf.

[Aya08] D. Ayala. Geometric Cobordism Categories. PhD thesis, Stanford University, 2008. arXiv preprint 0811.2280.

[Bae02] John Baez. The Octonions. Bull. Amer. Math. Soc., 39(2):145205, 2002.

[BDSPV15] B. Bartlett, C. L. Douglas, C. J. Schommer-Pries, and J. Vicary. Modular categories as representations of the 3-dimensional bordism 2-category. arXiv preprint 1509.06811, 2015.

[BM14] M. Bökstedt and I. Madsen. The cobordism category and Waldhausen's K-theory. Contemporary Mathematics - American Mathematical Society, 617:39-80, 2014. 
[BP64] E. H. Brown and F. P. Peterson. Relations among characteristic classes -I. Topology, 3:39-52, 1964.

[CM16] F. Cantero Morán. A metric for the space of submanifolds of Galatius and Randal-Williams. arXiv preprint 1512.06849, 2016.

[CMP15] F. Cantero Morán and M. Palmer. On homological stability for configuration spaces on closed background manifolds. Documenta Mathematica, 20:265-284, 2015.

[CMRW17] F. Cantero Morán and O. Randal-Williams. Homological stability for spaces of embedded surfaces. Geometry \& Topology, 21:1387-1467, 2017.

[CRS17] N. Carqueville, I. Runkel, and G. Schaumann. Line and surface defects in Reshetikhin-Turaev TQFT. arXiv preprint $1710.10214,2017$.

[DHH10] C. L. Douglas, A. G. Henriques, and M. A. Hill. Homological obstructions to string orientations. International Mathematics Research Notices, 18:4074-4088, 2010.

[Dol56] A. Dold. Vollständigkeit der Wuschen Relationen zwischen den Stiefel-Whitneyschen Zahlen differenzierbarer Mannigfaltigkeiten. Math. Z., 65(1):200-206, Dec 1956.

[FH16] D. S. Freed and M. J. Hopkins. Reflection positivity and invertible topological phases. arXiv preprint 1604.06527, 2016. 
[Gen12] J. Genauer. Cobordism categories of manifolds with corners. Transactions of the American Mathematical Society, 364(1):519$550,2012$.

[GMTW09] S. Galatius, I. Madsen, U. Tillmann, and M. Weiss. The homotopy type of the cobordism category. Acta Mathematica, 202(2):195-239, 2009.

[GRW] S. Galatius and O. Randal-Williams. Homological stability for moduli spaces of high dimensional manifolds. I. Journal of the American Mathematical Society (to appear).

[GRW10] S. Galatius and O. Randal-Williams. Monoids of moduli spaces of manifolds. Geometry \& Topology, 14:1243-1302, 2010.

[GRW14] S. Galatius and O. Randal-Williams. Stable moduli spaces of high-dimensional manifolds. Acta Mathematica, 212(2):257-377, 2014 .

[GRW17] S. Galatius and O. Randal-Williams. Homological stability for moduli spaces of high dimensional manifolds. II. Annals of Mathematics, 186(1):127-204, 2017.

[HL11] Y. Huang and N. C. Leung. A uniform description of compact symmetric spaces as Grassmannians using the magic square. Mathematische Annalen, 350(1):79-106, 2011.

[Hoe] R. S. Hoekzema. Personal website. https://people.maths.ox.ac.uk/hoekzema/Mathematics.html. 
[Hoe17] R. S. Hoekzema. Manifolds with odd Euler characteristic and higher orientability. Accepted for publication in International Mathematical Research Notices; arXiv preprint 1704.0660\%, 2017.

[Ish92] K. Ishitoya. Squaring operations in the Hermitian symmetric spaces. J. Math. Kyoto Univ., 32(1):235-244, 1992.

[Jac74] N. Jacobson. Basic Algebra I. W. H. Freeman and Company, 1974.

[Jon99] V. F. R. Jones. Planar Algebras, I. arXiv preprint 990902\%, 1999.

[Juh14] A. Juhász. Defining and classifying TQFTs via surgery. arXiv preprint 1408.0668, 2014.

[Koc03] J. Kock. Frobenius algebras and 2D topological quantum field theories. Cambridge University Press, 2003.

[Kra17] M. Krannich. Homological stability of topological moduli spaces. arXiv preprint 1710.08484, 2017.

[KS17] L. Kennard and Z. Su. On dimensions supporting a rational projective plane. Journal of Topology and Analysis, 2017. https://doi.org/10.1142/S1793525319500237.

[Lee03] J. M. Lee. Introduction to Smooth Manifolds, volume 218 of Graduate Texts in Mathematics. Springer New York, New York, NY, 2003. 
[Luc78] E. Lucas. Théorie des fonctions numériques simplement périodiques. American Journal of Mathematics, 1(4):289-321, 1878.

[Lur09] J. Lurie. On the classification of topological field theories. Current Developments in Mathematics, 2008:129-280, 2009.

[Mas60] W. S. Massey. On the Stiefel-Whitney classes of a manifold. American Journal of Mathematics, 82(1):92-102, 1960.

[May99] J. P. May. A Concise Course in Algebraic Topology. University of Chicago press, 1999.

[Mic80] P. W. Michor. Manifolds of differentiable mappings. Shiva Publishing Limited, 1980.

[MM02] C. Mantegazza and A. C. Mennucci. Hamilton-Jacobi Equations and Distance Functions on Riemannian Manifolds. Appl. Math. Optim., 47(1), 2002.

[MMASC14] M.G. Monera, A. Montesinos-Amilibia, and E. SanabriaCodesal. The Taylor Expansion of the Exponential Map and Geometric Applications. Revista de la Real Academia de Ciencias Exactas, Fisicas y Naturales. Serie A. Matematicas, 108(2):881906, 2014.

[MS74] J. W. Milnor and J. D. Stasheff. Characteristic classes (AM76). Princeton University Press, 1974. Annals of Mathematics Studies, No. 76. 
[Mun00] J. R. Munkres. Topology. Prentice Hall, Inc., 2000.

[Nak01] M. Nakagawa. The mod 2 cohomology ring of the symmetric space EVI. Journal of Mathematics of Kyoto University, 41(3):535-556, 2001.

[Och81] S. Ochanine. Signature modulo 16, invariants de Kervaire généralisés et nombres caractéristiques dans la K-théorie réelle. Mémoires de la Société Mathématique de France, 5:1-142, 1981.

[Per14] N. Perlmutter. Cobordism Category of Manifolds With BaasSullivan Singularities, Part 2. arXiv preprint 1306.4045, 2014.

[Per15] N. Perlmutter. Cobordism Category of Manifolds With BaasSullivan Singularities, Part 1. Münster Journal of Mathematics, $8(1), 2015$.

[Ran02] A. Ranicki. Algebraic and Geometric Surgery. Oxford University Press, 2002.

[Rud98] Y. B. Rudyak. On Thom spectra, Orientability, and Cobordism. Springer-Verlag, 1998.

[RW11] O. Randal-Williams. Embedded cobordism categories and spaces of submanifolds. International Mathematics Research Notices, 3:572-608, 2011.

[SE62] N. E. Steenrod and D. B. A. Epstein. Cohomology operations. Princeton University Press, 1962. 
[SP11] C. J. Schommer-Pries. The Classification of Two-Dimensional Extended Topological Field Theories. arXiv preprint 1112.1000, 2011.

[SP17] C. Schommer-Pries. Invertible Topological Field Theories. arXiv preprint 1712.08029, 2017.

[Sto63] R. E. Stong. Determination of $H^{*}\left(B O(k, \ldots, \infty), Z_{2}\right)$ and $H^{*}\left(B U(k, \ldots, \infty), Z_{2}\right)$. Transactions of the American Mathematical Society, 107(3):526-544, 1963.

[TW74] Hirosi Toda and Takashi Watanabe. The integral cohomology rings of $F_{4} / T$ and $E_{6} / T$. J. Math. Kyoto Univ., 14(2):257-286, 1974.

[Yos17] J. Yoshida. Transversality theorem in highly relative situations and its application. arXiv preprint 1703.02146, 2017. 